\title{
EFFECT OF SURFACTANT ARCHITECTURE ON CONFORMATIONAL TRANSITIONS OF CONJUGATED POLYELECTROLYTES
}

\author{
A Thesis \\ presented to \\ the Faculty of California Polytechnic State University, \\ San Luis Obispo \\ In Partial Fulfillment \\ of the Requirements for the Degree \\ Master of Science in Polymers and Coatings Science
}

by

Gregory Braggin

June 2015 
(C) 2015

Gregory Braggin

ALL RIGHTS RESERVED 


\section{COMMITTEE MEMBERSHIP}

TITLE:

AUTHOR:

DATE SUBMITTED:

COMMITTEE CHAIR:

COMMITTEE MEMBER:

COMMITTEE MEMBER:
Effect of Surfactant Architecture on Conformational

Transitions of Conjugated Polyelectrolytes

Gregory Braggin

June 2015

Shanju Zhang, Ph.D.

Assistant Professor of Chemistry

Philip Costanzo, Ph.D.

Associate Professor of Chemistry

Chad Immoos, Ph.D.

Associate Professor of Chemistry 


\begin{abstract}
Effect of Surfactant Architecture on Conformational Transitions of Conjugated Polyelectrolytes

Gregory Braggin
\end{abstract}

Water soluble conjugated polyelectrolytes (CPEs), which fall under the category of conductive polymers, possess numerous advantages over other conductive materials for the fabrication of electronic devices. Namely, the processing of water soluble conjugated polyelectrolytes into thin film electronic devices is much less costly as compared to the processing of inorganic materials. Moreover, the handling of conjugated polyelectrolytes can be performed in a much more environmentally friendly manner than in the processing of other conjugated polymers because conjugated polyelectrolytes are water soluble, whereas other polymers will only dissolve in toxic organic solvents. The processing of electronic devices containing inorganic constituents such as copper indium gallium selenide (CIGS), is much more expensive and poses much greater environmental risks because toxic metals may be released into landfills or waterways upon cell disposal. ${ }^{75}$ Because conjugated polyelectrolytes enjoy an assortment of advantages over other materials for the manufacturing of thin film electronic devices, there is globally vested interest in the researching of their properties. Despite the fact that CPEs can serve as efficient electron transport mediums, devices such as organic solar cells cannot realize their highest efficiencies unless the morphology of CPEs is precisely controlled. Charged surfactants can electrostatically and ionically interact with CPEs, and when introduced in 
specific concentrations, molar ratios, and temperature ranges, will aid in a 'coil to rod' transition of the CPE, wherein polymer chains undergo intramolecular transitions to obtain rigid-rod morphologies. The kinetics and thermodynamics of the 'coil to rod' transition are heavily dependent upon the type(s) of charged surfactant complexed with the CPE (i.e. on the surfactant architecture). By performing UV/Vis Spectroscopy and Fluorometry on dilute polymer/surfactant solutions, Polarized Optical Microscopy (POM) and Small Angle X-Ray Scattering (SAXS) on high concentration polymer/surfactant solutions, and Differential Scanning Calorimetry (DSC) and X-Ray Diffraction (XRD) on solid-state polymer/surfactant samples, the role of various surfactant architectures on the kinetics and thermodynamics of the 'coil to rod' transition was studied. The liquid crystalline physical properties and the extent of solid state crystallinity were also investigated. Through an analysis of the data obtained from these various techniques, it was found that the 'coil to rod' transition is progressively favored when the alkyl chain length of a single tailed surfactant is sequentially increased, and that as the concentration of double-tailed surfactant increases, the 'coil to rod' transition is negated. 


\section{ACKNOWLEDGMENTS}

Performing successful scientific research requires frequent consultation with others, as well as recurrent mentoring. Without the guidance of the faculty at Cal Poly, I would definitely not have made as much progress as I did in my research. In particular, I would like to thank Dr. Zhang for his ample availability, concern for student development, and willingness to listen to newly discovered findings or challenges. Dr. Zhang offered intriguing perspectives on research that allowed for a graduate student to

greatly enhance their analytical skills. By mentoring me through my research project, Dr. Zhang allowed me to become a well-trained and critically thinking scientist. His widespread knowledge of Chemistry and Materials Science was always evident, and I appreciate the time that he took to discuss meaningful data and relevant academic publications with me.

Secondly, I would like to thank Dr. Costanzo for his time and efforts on my behalf. Dr. Costanzo was very supportive and encouraging throughout my research endeavor; whenever I had a question about a particular phenomenon or whenever I was excited by certain principles within science, he was more than willing to share his knowledge with me. In both the successful and challenging periods of my research, Dr. Costanzo encouraged me to keep working hard and to remain inspired by the rewards of research, which can occur at the most unexpected of times.

I would also like to thank Dr. Immoos for serving on my thesis committee. Dr. Immoos remained genuinely interested about the progress that I was making with Dr. 
Zhang throughout my research project, as he listened attentively to the numerous findings of the research and also offered insight into possible future experiments that could move the research even further. Lastly, I would like to thank Dr. Fernando, the ACS Petroleum Research Fund, the Bill Moore Fellowship, the Cal Poly departments of Chemistry and Biochemistry and Materials Engineering, and the Polymers and Coatings Program. The support I received from the individuals affiliated with these organizations made it possible to have the resources necessary for pursuing efficient research that was both meaningful and enjoyable. 


\section{TABLE OF CONTENTS}

LIST OF TABLES $\mathrm{X}$

LIST OF FIGURES $\mathrm{xi}$

\section{CHAPTER}

1. INTRODUCTION

1.1 History of Conductive Polymers .............................................................

1.2 The Mechanisms of Conductivity in Conjugated Polymers ................................4

1.3 Tailoring the Morphology of Conjugated Polymers .........................................12

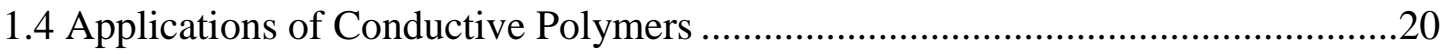

1.5 Liquid Crystalline Polymers and Their Applications .......................................25

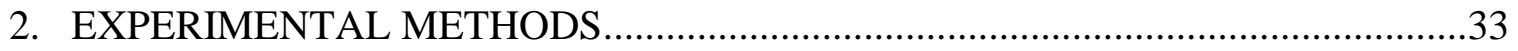

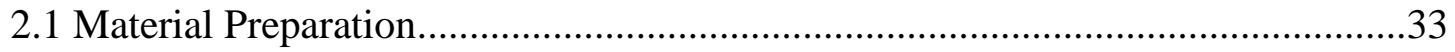

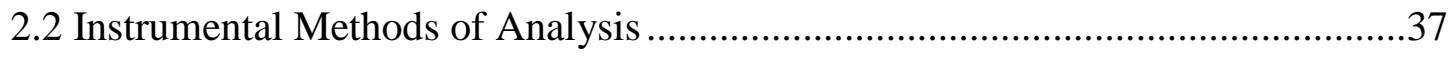

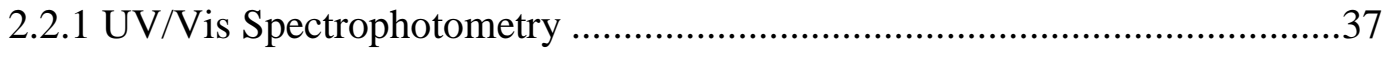

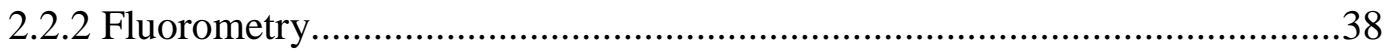

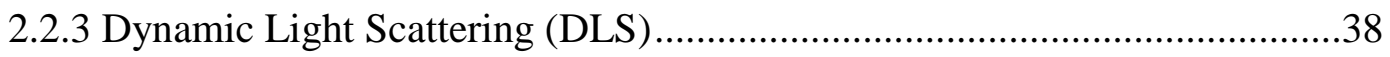

2.2.4 Polarized Optical Microscopy (POM) ...................................................39 
2.2.5 Differential Scanning Calorimetry (DSC)

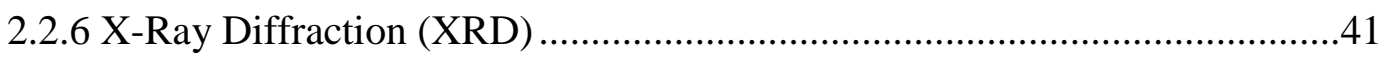

2.2.7 Small-Angle X-Ray Scattering (SAXS) …………...................................42

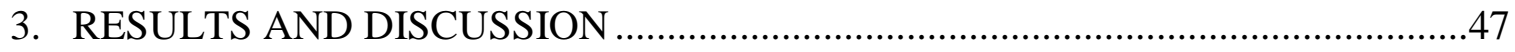

3.1 Dilute Solution Spectroscopy ………………………....................................4

3.2 Dynamic Light Scattering (DLS) Studies ..........................................................66

3.3 Properties of Concentrated Solutions................................................................

3.4 Properties of Solid State Complexes....................................................................

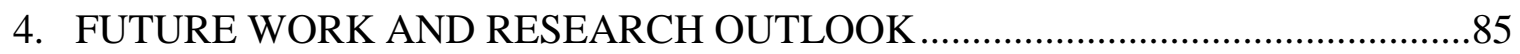

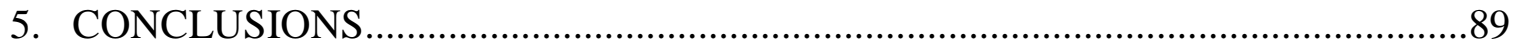

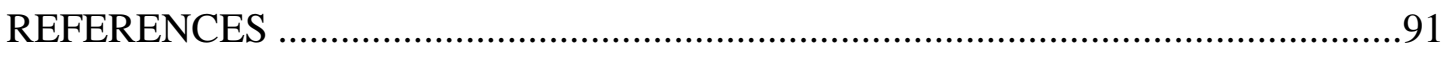




\section{LIST OF TABLES}

Table

Table 1: Photovoltaic Device Efficiencies for $\mathrm{TiO}_{2} /$ Polymer Solar Cells

Processed from Various Solvents. ${ }^{24}$

Table 2: Various Applications of Liquid Crystalline Polymers. ${ }^{44}$

Table 3: Approximate Bandgaps in eV of a P3KHT/CTAB Complex

at Various Times (Minutes) from Figure 3.2.

Table 4: Mean Particle Size and Standard Deviation for 13.3 $\mu$ M Solutions of Various

Complexes as Determined via Dynamic Light Scattering (DLS). 


\section{LIST OF FIGURES}

Figure

Page

Figure 1.1: $p T T B A$ dye incorporated into a dye sensitized solar cell device,

followed by an illustration of the molecular structures of the

respective HOMO and LUMO levels. ${ }^{23}$

Figure 1.2: Conjugated polymers may exhibit electrical performance

as insulators or as very strong conductors like transition metals. ${ }^{8}$

Figure 1.3: Charge transport mechanism and energy band structure for a conjugated

polymer (polypyrrole).

Figure 1.4: An illustration of the stacking between adjacent

conjugated polymer chains.

Figure 1.5: A SrYCoO 2 crystal structure.

Figure 1.6: The doping of polyacetylene.

Figure 1.7: poly(anthraquinonyl sulfide) (PAQS) being doped with a reducing agent $\left(\mathrm{Na}^{+}\right)$, leading to polyelectrolyte formation and charge transport capabilities. ${ }^{9}$ .11

Figure 1.8: The doping of polyaniline emraldine base with $\mathrm{HCl}$

to produce a polymeric radical cation. 
Figure 1.9: A photovoltaic device based on a $\mathrm{TiO}_{2}$ (acceptor) and PTEBS (donor) heterojunction.

Figure 1.10: The magnetically induced alignment of a polymeric entity. .17

Figure 1.11: Electric Field and Orientation. .18

Figure 1.12: Structure of a polymer solar cell with bulk heterojunction (BHJ) morphology.

Figure 1.13: Interface between donor and acceptor domains.

Figure 1.14: Illustration of conjugated polymer conformational

changes upon an increase in concentration. .26

Figure 1.15: Sample birefringence.

Figure 1.16: Phase sequence of liquid crystalline polymers

for increasing temperature. .28

Figure 2.1: Molecular Structures. .34

Figure 2.2: SAXS Patterns.

Figure 2.3: A conventional SAXS instrumental setup. ${ }^{48}$ .44

Figure 2.4: Determination of Radius of Gyration of polymer particles from SAXS data. ${ }^{48}$ .45

Figure 3.1: A conventional absorbance spectra. .49 
Figure 3.2: Absorbance vs. wavelength (nm) and time dependent chromism of a

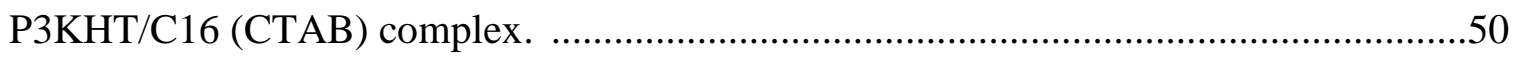

Figure 3.3: Hypothetical polymer/surfactant conformations as a function of time. .52

Figure 3.4: Time dependent chromism of a P3KHT/5\% DHAB Complex. ' $\mathrm{t}$ ' is time in minutes.

Figure 3.5: UV/Vis spectra of a P3KHT/10\% DHAB solution. .57

Figure 3.6: UV/Vis spectra of 10, 20, and 30\% DHAB complexes. .58

Figure 3.7: Emission spectra for a P3KHT/5\% DHAB solution. .60

Figure 3.8: Normalized Emission spectra for 10, 20, and 30\% DHAB/P3KHT solutions. .61

Figure 3.9: Normalized Absorbance spectra for P3KHT/C10, C12, C14, and C16 solutions. .64

Figure 3.10: Normalized Emission Intensity for P3KHT/C10, C12, C14, and C16 solutions. .66

Figure 3.11: Mean particle size vs. alkyl chain length. There is a reduction in particle size for chains greater than 14 carbons. .68

Figure 3.12: Mean particle size vs. percent DHAB surfactant in system. .69

Figure 3.13: Phase behavior of P3KHT/Surfactant solutions as a function of concentration. ${ }^{66}$ .71 
Figure 3.14: Illustration of the Nematic, Biphasic, and Liquid Crystal Phases

before and after rotation of polarized light.

Figure 3.15: Other illustrations of the Biphasic Phase.

Figure 3.16: Depiction of other attainable liquid crystalline

and solid film textures.

Figure 3.17: Experimentally determined phase transitions for P3KHT complexes of varying DHAB loading ratios.

Figure 3.18: Experimentally determined phase transitions for P3KHT complexes of varying surfactant alkyl chain length. .78

Figure 3.19: X-Ray Diffractogram of a P3KHT/Surfactant complex of varying

DHAB concentrations. ${ }^{66}$ .80

Figure 3.20: DSC spectra of P3KHT/Surfactant

complexes of varying alkyl chain length.

Figure 3.21: DSC spectra of P3KHT/Surfactant complexes

of varying DHAB loading ratios. ${ }^{66}$ .82

Figure 3.22: Polarized Optical Microscope (POM) Images of

Dried Polymer/Surfactant Film. .84 


\section{Introduction}

\subsection{History of Conducting Polymers}

For decades, many in the field of Chemistry had doubted the possibility of synthesizing long chain macromolecules and had queried the existence of conducting organic molecules. In 1920, a letter was written to Hermann Staudinger refuting his belief that a macromolecule could exist, as Staudinger had made a prior claim that rubbers, starches, and cellulosic entities were long chains of short repeating molecular units connected by covalent bonds. ${ }^{1}$ Many were skeptical of the findings within the field of polymer science during the mid- $20^{\text {th }}$ century and could not fathom how organic long chain molecules could conduct electricity. In the 1970s, however, conducting organic molecular crystals were discovered when planar molecules with delocalized systems of $\pi$ electrons yielded noticeable conductivity. TTF-TCNQ (tetrathiofulvalene-7, 7, 8, 8tetracyano- $p$-quinodimethane) was one of the first organic materials to have recognizable conductivity, due to its electron deficiency and the ease with which it could form an anion radical. A charge transfer complex formed when TTF (a donor) was bonded to TCNQ (an acceptor); TTF-TCNQ was also found to crystallize into independent stacks, wherein a three dimensional charge transfer network could be created. ${ }^{2}$ An even larger breakthrough in the discovery of conducting organic molecules came in the late 1970s when Hideki Shirakawa et. al. found that polyacetylene could become highly conductive upon incorporation of electron donating or accepting molecules. ${ }^{3}$

Shirakawa and coworkers Alan G. MacDiarmid and Alan J. Heeger worked to better understand how a polymer could deliver marked increases in conductivity upon the 
introduction of dopants. After further study, it was found that the alternation between single and double bonds led to a delocalization of electron density in between adjacent $p$ orbitals, commonly referred to as a $\pi$-bond. A dopant is analogous to a charge carrier in that mobile electrons or holes are created when a dopant is injected into a material. A 'hole' is simply a formalism describing a site that has an absence of an electron. When an electron from a neighboring position moves to fill a hole, a new hole is simultaneously created and charge thus migrates automatically across long distances ${ }^{3}$ — this effect caused by doping caught the attention of many researchers. MacDiarmid decided to investigate in detail the effects of doping on conductive properties and found that by adding small amounts of iodine to polyacetylene, conductivity could be enhanced up to sevenfold. By tailoring the ratio of cis/trans bonds, better orientations of the polymer chains could be realized and even greater conductivities attained. By the early 1980s, other polymers such as polypyrrole, polythiophene and associated derivatives, polyphenylenevinylene, and polyaniline were discovered and proved to have noteworthy conductivities. Polythiophene and polypyrrole differ from polyacetylene in that their conductivities are not quite as high as polyacetylene, yet they are still viable for producing flexible organic electronic devices. ${ }^{3}$

In the present day, thin film electronics and biosensors are a few amongst a plethora of different applications for conjugated polymers. Further research has led to the optimization of the mechanical flexibility and thermal stability of conductive polymers, and as such, they have been successfully implemented into devices such as light emitting diodes (LEDs) and supercapacitors. ${ }^{4}$ Large area films of conductive polymers are routinely synthesized during the modern era via electrochemical or other synthetic 
techniques such as Kumada catalyst transfer polymerization or Grignard metathesis polymerization. ${ }^{5}$ Continuous flow synthetic methods of conductive polymers are becoming increasingly favored over regular batch methods, as rapid synthesis has been achieved in industrial settings through Suzuki-Miyaura and Stille coupling reactions wherein molecular weight and regioregularity can be tightly controlled. Due to the facile large scale synthesis methods that have been developed, the scaling up of devices containing conductive polymers has occurred at unprecedented rates and production costs have dwindled. ${ }^{5}$ In large measure, the development of low cost methods for synthesizing conductive organic macromolecules has led to the advent of organic-based electronic devices that are beginning to rival both the cost and durability of inorganic analogues. ${ }^{81}$

Polymer incorporation into organic electronic devices is considered to be a necessary step in the device integration process; in modern day dye-sensitized solar cells (DSSCs), conductive polymers are employed as electron transfer mediators or as photoreceptors to enhance device efficiency. ${ }^{6}$ The heavy weight, high cost, and lack of flexibility associated with silicon or other inorganic based solar cells have compelled researchers to investigate the properties of several conductive polymers currently employed in solar cells, biosensors, biofuel cells, LEDs, and a variety of other electronic devices. Poly 5, 2': 5', 2'-terthiophene-3'-carboxylic acid (poly-TTCA), poly 3'-cyano5,2': 5',2"'terthiophene (poly-CTT), and poly 3',4-diamino-2,2': 5',2”-terthiophene (poly-DATT) are a few of many polymeric samples to have demonstrated photosensitizing capabilities superior to inorganic materials such as ruthenium. Furthermore, polymer structures may be modified through synthesis techniques to reduce steric hindrance and to establish greater effective conjugation lengths, ultimately leading 
to the enhancement of electron transferring capabilities. ${ }^{6}$ In Figure 1.1, a [2,2': 5', 2"'terthiophene-3' $(p$-benzoic acid)] ( $p$ TTBA) conductive polymer dye is illustrated as being incorporated into a dye sensitized solar cell device, wherein it is in contact with an $\mathrm{I}_{3}{ }^{-} / \mathrm{I}^{-}$ electrolyte, which may serve as a dopant.

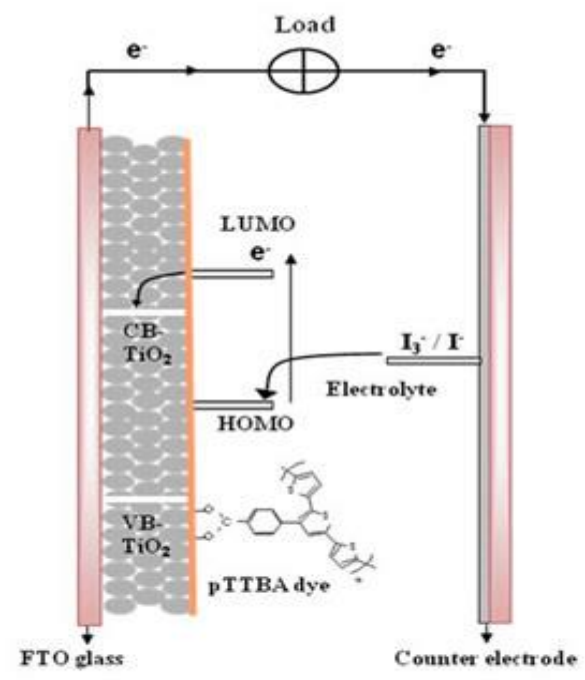

(A)

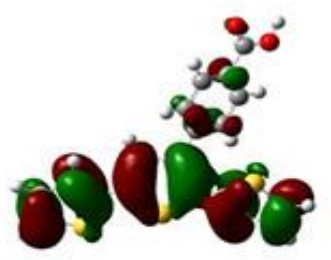

HOMO

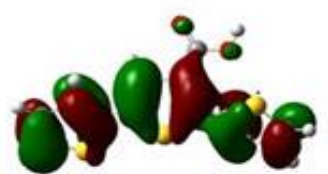

HOMO

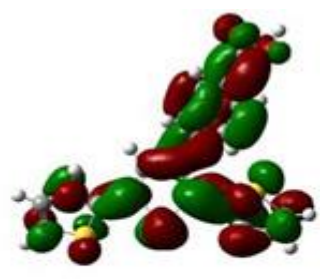

LUMO

(a) TTBA

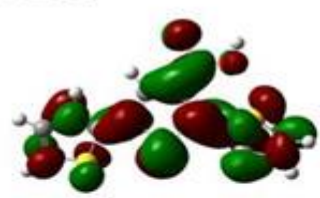

LUMO (b) TTCA

(B)

Figure 1.1: $p T T B A$ dye incorporated into a dye sensitized solar cell device, followed by an illustration of the molecular structures of the respective HOMO and LUMO levels. ${ }^{23}$

\subsection{The Mechanisms of Conductivity in Conjugated Polymers}

One must understand that the electron delocalization along adjacent $p$-orbitals is only one of several factors governing the conductivity of polymers. Regardless of charge delocalization due to the network of $\pi$-bonds along a polymeric backbone, charge transport will not be facile unless specific morphologies are attained. The ease of charge 
transport is also dictated by variables such as doping levels; the effects of even a minor amount of doping are substantial, as conductivity can increase from $10^{-10} \mathrm{~S} / \mathrm{cm}$ to greater than $0.1 \mathrm{~S} / \mathrm{cm}$ at less than a $1 \mathrm{wt} . \%$ doping concentration. ${ }^{7}$ The energy level difference between the ground and excited state within a macromolecule such as a polymer is referred to as a bandgap between the valence band (lower energy state) and conduction band (higher energy state), whereas in a single molecule the lower energy state is the highest occupied molecular orbital (HOMO) and the higher energy state is the lowest unoccupied molecular orbital (LUMO). The bandgap of a polymer is substantially reduced upon doping in that electrons are removed from $p$-orbitals upon oxidation, and as such, electrons from adjacent $p$-orbitals will travel to fill in the vacancies, leading to a 'one-dimensional electronic band.' In the 'one-dimensional electronic band,' charge mobility increases as vacancies are created. When the polymeric bandgap is lowered, the length of the rigid backbone referred to as an effective conjugation length (ECL) increases, wherein charge mobility is not impeded by kinks or traps. In Figure 1.2, the wide range of conductivities which a polymer may assume are listed.

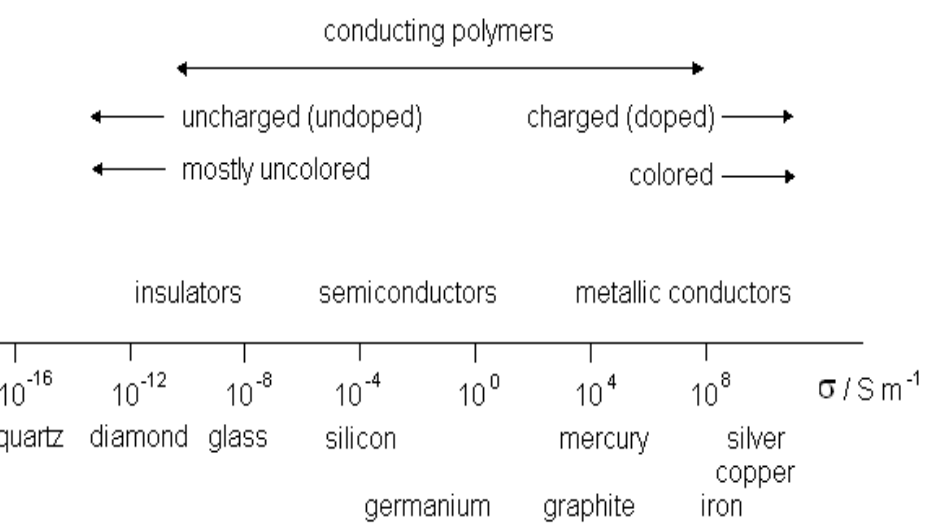

Figure 1.2: Conjugated polymers may exhibit electrical performance as insulators or as very strong conductors like transition metals. ${ }^{8}$ 
The conductivity of a conjugated polymer depends heavily on the doping level, as well as on the chemical identity of introduced dopants. Different dopants have dissimilar oxidation potentials, and when a certain chemical potential is applied, 'electron hopping' can easily occur along a polymeric backbone, leading to enhanced conductivity and optoelectronic performance. In an insulator, there is a very large bandgap between the valence and conduction bands, and in polymers with poor conductivity, the bandgap may be as high as $7.0 \mathrm{eV} .{ }^{9}$ The binding energy of electrons is much higher in insulators than in conductors, as valence electrons tend to possess much more attraction towards the nucleus as compared to traditional metal conductors, where valence electrons in the $d$ orbitals are well removed from the atomic center. As is implied in Figure 2, conjugated polymers which are insulating may be made conductive through chemical doping, but the charge transport will not be continuous across three dimensions as it is for inorganic conductors. Instead, charge transport will only occur across one dimension for a conjugated polymer. Despite this limitation, charge transport can be as much as 100 times greater along the backbone of chains versus transport in between adjacent chains. Because charge transport is a one dimensional process in conjugated polymers, it is imperative to attain chain morphologies which are as straight as possible to mitigate charge trapping and annihilation effects. In Figures 1.3, 1.4, and 1.5, the molecular arrangements of the orbitals responsible for charge transport are depicted for conjugated polymers and inorganic conductors, respectively, as are the corresponding charge transport mechanisms. 


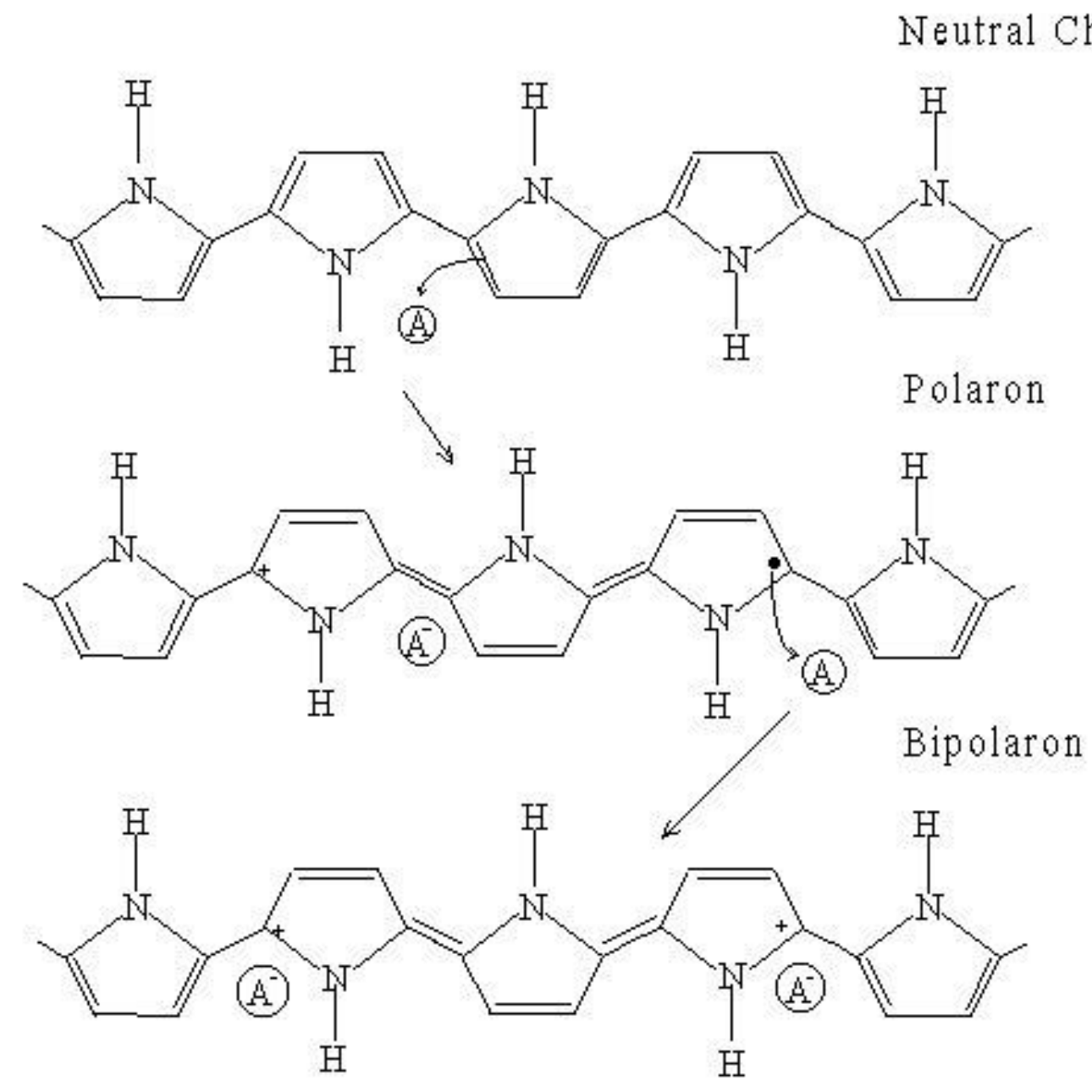

Figure 1.3: Charge transport mechanism and energy band structure for a conjugated polymer (polypyrrole). Oxidation occurs upon doping, leading to the production of a polaron or a bipolaron. Upon heavy doping, continuous bipolaron bands may be formed, and over time, a merging of the upper and lower bipolaron bands may occur with the valence and conduction bands, leading to partially filled bands and metallic like conductivity. ${ }^{10}$

As will be discussed in the latter portions of this report, $\pi-\pi$ stacking contributes to strong electrostatic interactions between adjacent polymer chains, as well as to noteworthy aggregation behavior. Depending on the molecular structure of the polymer 
and the surrounding environment (i.e. the solvent), $\pi-\pi$ stacking may result in morphologies which do not favor charge transport, and as a consequence, several strategies are typically employed to tailor the polymer chain morphology to optimize charge transport. By applying electric or magnetic fields, mechanical shear force, or by introducing surfactant, the aggregation behavior due to the electrostatic $\pi-\pi$ interactions can be mitigated and chains may more easily assume orientations with greater effective conjugation lengths (ECLs). In inorganic conductors, there is less of a necessity to precisely tune the morphology of one dimensional moieties such as polymer chains, as the three dimensional crystal structure typically allows for robust charge mobility. In Figure 1.5, a unit cell crystal structure of $\mathrm{SrYCoO}_{2}$ is depicted to demonstrate a three dimensional inorganic conductive network possessing a high degree of crystallinity.

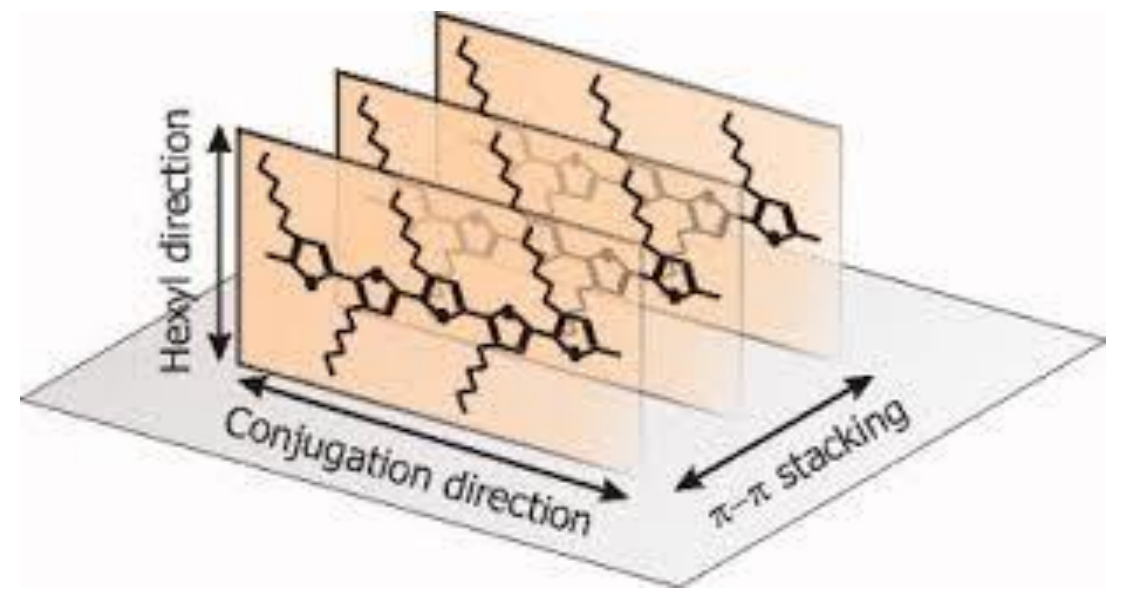

Figure 1.4: An illustration of the stacking between adjacent conjugated polymer chains. This stacking may allow for an aligned network to form (under certain circumstances), thus contributing to morphologies which favor charge transport. ${ }^{11}$ 


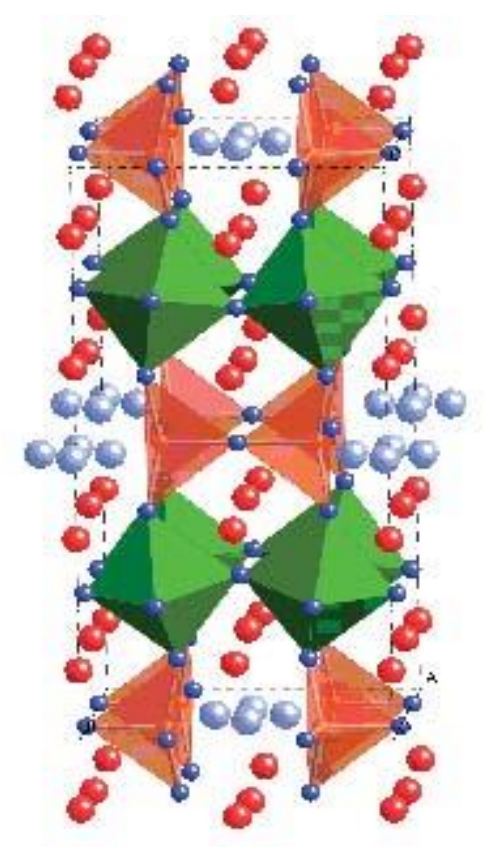

Figure 1.5: A SrYCoO 2 crystal structure. In inorganic conductors, a three-dimensional crystal network exists, leading to charge transport capabilities in all directions. ${ }^{12}$

Nearly all conjugated polymers can be oxidized (p-type doping) or reduced (ntype doping) via the introduction of electron acceptors or donors. Introducing an oxidizing agent such as iodine to a conjugated polymer like polyacetylene may lead to an increase in conductivity of about $10^{-5}$ to $10^{-2} \mathrm{~S} / \mathrm{cm} .{ }^{14}$ The doping mechanism is depicted in Figure 1.6. 


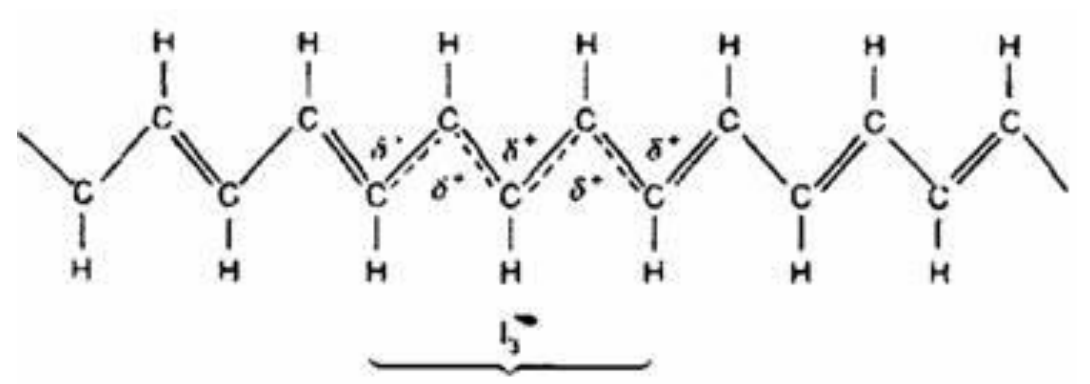

Figure 1.6: The doping of polyacetylene. An electron-hole pair is created upon doping with $\mathrm{I}_{3}{ }^{-}$(indicated by the dotted lines, wherein electron hopping is most likely to occur). ${ }^{13}$

In Figure 1.6, one can notice the delocalization of the double bonds induced by iodine doping. The creation of an electron-hole pair or 'polaron' has led to increased charge mobility based on the rational of empty electron orbitals being continuously filled via the 'charge hopping' mechanism discussed previously. Analogously, conductive polymers may be doped with electron donors to improve their charge transport capabilities. In Figure 1.7, poly(anthraquinonyl sulfide) (PAQS) is doped with $\mathrm{Na}^{+}$ions from a $\mathrm{Na}$ based electrolyte. In modern applications, PAQS serves as a polymeric cathode or anode, and due to the ease with which it can be doped with $\mathrm{Na}^{+}$electrochemically, low cost organic batteries are evolving within the marketplace. ${ }^{9}$ Charge storage capacities and charge transfer reaction capabilities have improved as a result of the PAQS n-type doping scheme. 

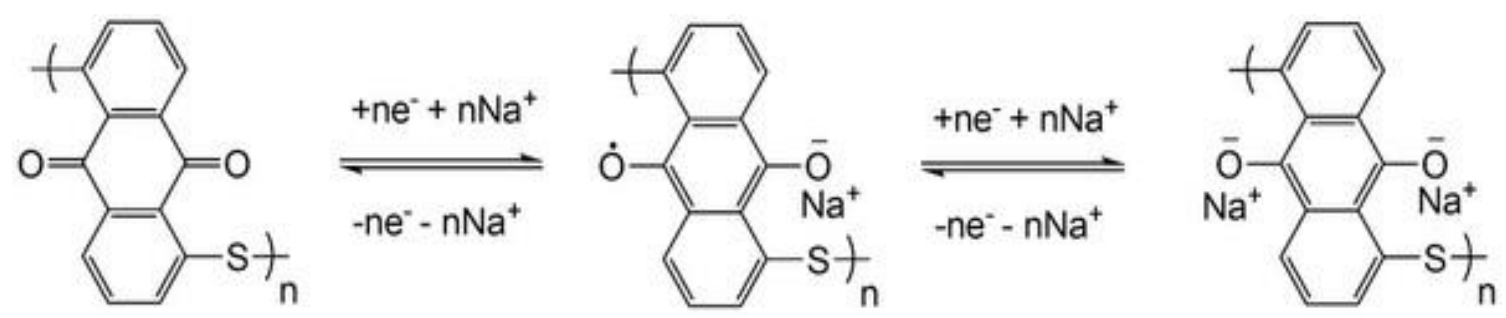

Figure 1.7: poly(anthraquinonyl sulfide) (PAQS) being doped with a reducing agent $\left(\mathrm{Na}^{+}\right)$, leading to polyelectrolyte formation and charge transport capabilities. ${ }^{9}$

Conjugated polymers may also be easily oxidized or reduced via other means such as electrochemical doping — by applying a DC power source between a positive electrode coated with conjugated polymer and a standard negative electrode such as graphite, doping can easily occur. It is necessary to fully immerse the positive and negative electrodes in an electrolyte solution that favors charge transport, such as $\mathrm{LiClO}_{4}$. A downside to electrochemical doping is that counter ions must be present in the solution in order to mitigate charge buildup; these counterions may cause distortions along the polymer backbone and may impede conductivity. ${ }^{9}$ In addition to doping polymers electrochemically, other methods such as photodoping may be employed. Electrons may be promoted from the valence band into the conduction band when a photon of sufficient energy is incident upon the polymer. Moreover, a potential difference may be applied during the irradiation to effectively separate electrons from holes, thus enhancing conductivity. ${ }^{9}$ One advantage of photodoping is that counter ions need not be introduced, and as a result, little distortion of the polymeric backbone or of the overall morphology occurs. Lastly, 'non-redox' doping may be employed as a means to enhance the conductivity of a conjugated polymer. In 'non-redox' doping, the bandgap of the polymer is the only parameter altered; there are no changes with respect to the number of electrons 
present along the polymer backbone. Polyaniline emraldine base (PANI-EB) may be protonated with aqueous protonic acids such as $\left(\mathrm{C}_{6} \mathrm{H}_{5}\right) \mathrm{SO}_{3} \mathrm{H}$ or $\mathrm{HCl}$ to produce conducting radical cations. ${ }^{14}$ Figure 1.8 provides an example of a polyaniline emraldine base being protonated with $\mathrm{HCl}$, leading to the subsequent production of a conjugated polyelectrolyte of enhanced conductivity.

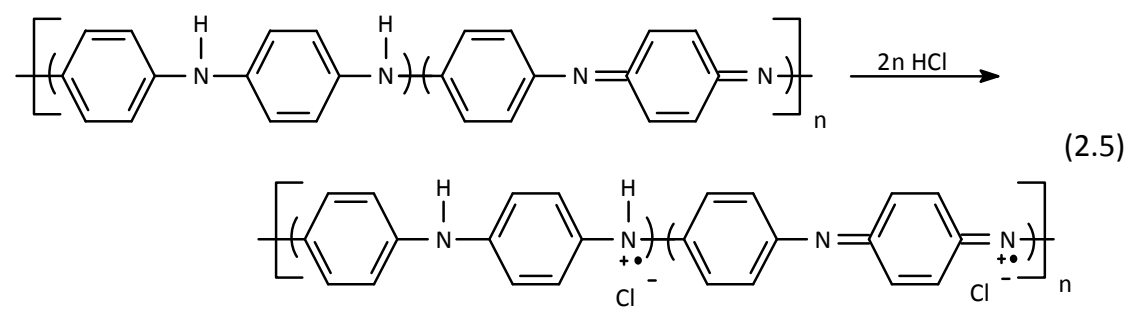

Figure 1.8: The doping of polyaniline emraldine base with $\mathrm{HCl}$ to produce a polymeric radical cation. This 'non-redox' doping strategy is a very popular means to enhance backbone charge transport. ${ }^{21}$

\subsection{Tailoring the Morphology of Conjugated Polymers}

As mentioned earlier, the performance of polymeric electronic devices relies primarily upon the ability for the backbone morphology to assume a rigid and aligned conformation. Past research has confirmed certain factors governing the morphological behaviors of conjugated polymers, such solvent type, environment, and packing density. In a 'good' solvent, conjugated polymer chains will have an affinity to associate with the solvent chiefly due to similarities in polarity. Polymer chains will expand in a 'good' solvent due to the favorable thermodynamic interactions between the polymer and solvent; as such, the intermolecular interactions between individual polymer chains will be reduced and the chains will expand into a 'coiled' morphology. The choice of solvent 
will also play a crucial role in influencing the performance of organic photovoltaic devices, as the morphologies and thermal stabilities of the polymeric entities depend heavily upon the type of solvent utilized. Solvents such as $o$-dichlorobenzene (ODCB) or chlorobenzene (CB) have been observed to induce diffusion of phenyl-C61-butyric acid methyl ester (PCBM) nanodomains with poly(3-hexylthiophene-2,5-diyl) (P3HT) to enhance device efficiency, where PCBM and P3HT are electron acceptors and donors, respectively. ${ }^{22}$ Because ODCB is a relatively non-polar solvent, it will only partially dissolve conjugated polyelectrolytes such as poly[3-(potassium-6-hexanoate)thiophene2,5-diyl] (P3KHT), and as such, it is deemed a 'poor' solvent. Thermodynamically, it is more favorable for the P3KHT chains to adopt a rod-like morphology in a 'poor' solvent as compared to an expanded coiled morphology; past studies have demonstrated that higher fractions of microcrystals with ordered conformations are promoted in poorer solvents. ${ }^{76}$ In essence, the intermolecular interactions between individual P3KHT chains are more favorable energetically than are the interactions between P3KHT and the 'poor' solvent. In the solution based processing of organic photovoltaic devices, the choice of solvents can dictate overall device performance, and 'poor' solvents can aid in the formation of self-assembled rod-like morphologies of polymer chains. In reality, however, only a few solvents are feasible for usage in device fabrication because factors such as toxicity and cost rule out the use of most solvents.

Water can be classified as a usable solvent for solution based processing of organic photovoltaic devices. Water is both polar and readily available, and under normal conditions, it is deemed as a 'good' solvent for P3KHT. Due to the widespread availability and low toxicity of water, large area thin film solar cells can be fabricated via 
liquid based processing — spin coating, doctor blading, ink jet printing, screen printing, and reel-to-reel processing are a few of the many device assembly techniques possible with water as a solvent. ${ }^{23}$ Time and again, water has demonstrated to be a viable solvent for the solution based processing of organic photovoltaic devices. In a study by Qiao et. al., the water soluble polymer PTEBS and anatase $\mathrm{TiO}_{2}$ nanocrystals were blended together and spin coated onto a fluorine doped tin oxide (FTO) substrate, serving as the cathode. A gold contact was evaporated on top of the $\mathrm{PTEBS} \mathrm{TiO}_{2}$ layer to serve as the anode and a voltage was applied between the anode and cathode. The study found that the efficiency of the device based on water processing rivaled the efficiencies of devices that had been processed from organic solvents. In Table 1, device efficiencies are listed for cells that were processed from different solvents, and in Figure 1.9, a schematic is provided of the PTEBS/TiO 2 based solar cell.

Table 1: Photovoltaic Device Efficiencies for $\mathrm{TiO}_{2} /$ Polymer Solar Cells Processed from Various Solvents. ${ }^{24}$

\begin{tabular}{|c|c|c|c|c|c|}
\hline Polymer & $\mathrm{J}_{\mathrm{SC}}\left(\mathrm{mA} / \mathrm{cm}^{2}\right)$ & $V_{O C}(V)$ & FF & $\eta(\%)$ & Ref. \\
\hline $\begin{array}{c}\text { P3UBT } \\
\text { (Solvent-based) }\end{array}$ & 0.45 & 0.67 & 0.29 & 0.10 & (69) \\
\hline $\begin{array}{c}\text { MEH-PPV } \\
\text { (Solvent-based) }\end{array}$ & 0.40 & 1.1 & 0.42 & 0.18 & (70) \\
\hline $\begin{array}{c}\text { TPD(4M)- } \\
\text { MEH-M3EH- } \\
\text { PPV } \\
\text { (Solvent- } \\
\text { Based) } \\
\end{array}$ & 0.96 & 0.86 & 0.50 & 0.41 & (71) \\
\hline $\begin{array}{c}\text { P3HT } \\
\text { (Solvent-based) }\end{array}$ & 2.76 & 0.44 & 0.36 & 0.42 & (72) \\
\hline $\begin{array}{c}\text { PTEBS (Water- } \\
\text { based) }\end{array}$ & 0.17 & 1.0 & 0.8 & 0.17 & (73) \\
\hline
\end{tabular}




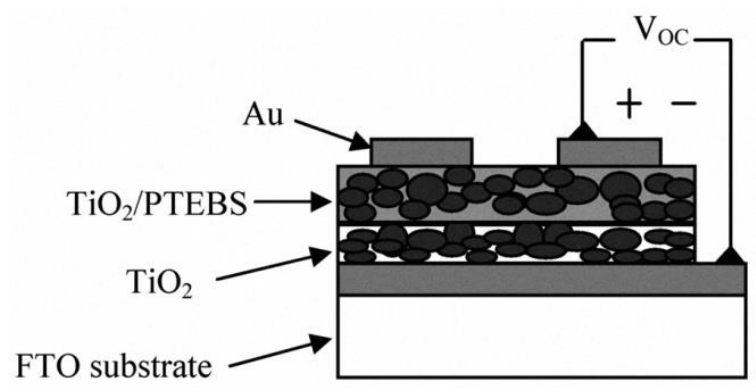

\section{Figure 1.9: A photovoltaic device based on a $\mathrm{TiO}_{2}$ (acceptor) and PTEBS (donor)}

heterojunction. The device was processed from a water based solution. ${ }^{63}$

When P3KHT is first dissolved in water, the anionic carboxylate moieties of P3KHT interact electrostatically with the dipole moment of water, and the P3KHT chains exist in the coiled form. The P3KHT chains are also aggregated with respect to one another based on $\pi-\pi$ stacking interactions. This aggregation behavior is evidenced by the lack of measurable fluorescent intensity, as intermolecular charge transfer yields nonradiative responses to excitation. ${ }^{24,25}$ Because water is a 'good' solvent for conjugated polyelectrolytes such as P3KHT, rod-like morphologies necessary for optimum photovoltaic device performance will not be attained unless various methods are utilized to modify the morphology. The use of charged surfactants can aid drastically in tuning the morphology of the coiled polymer chains in water, and hence water can remain a feasible and cost effective solvent for device processing. Surfactants in effect serve as 'morphology modifiers' because conformational transitions of the polymer chains may occur in their presence. The so called 'coil to rod' transition upon surfactant addition is a phenomenon that has garnered the attention of polymer scientists for many years in that the formation of microphases and gels can occur, accompanied by a modification of 
rheological and optoelectronic properties. ${ }^{27}$ The transition of individual polymer chains from coiled to rod form does not occur above certain concentrations, as excessive electrostatic repulsions prevent a continuous network of aligned chains. Moreover, the rod-like morphology will not occur above a certain temperature, treating all other variables as fixed, because the increased entropy disrupts the intermolecular interactions between chains. Based on the Gibbs free energy relationship of $\Delta G=\Delta H-T \Delta S$, the increase in entropy upon solution heating outweighs the enthalpic cost of disrupting the aligned rod network, and the coiled morphology is thermodynamically favored. Lastly, the architecture of the surfactant plays a crucial role in dictating the assumed morphology of the polymer chains. In general, single chain cationic surfactants with a longer alkyl backbone chain length will favor the formation of rod-like morphology. When a single tail cationic surfactant such as cetyltrimethylammonium bromide (CTAB) is combined with a double-tail cationic surfactant such as dihexadecyldimethylammonium bromide (DHAB), rod-like morphology is only favored for very high molar ratios of CTAB: DHAB. $^{51}$

In addition to using surfactant for tuning the morphology of conjugated polymers for electronic applications, electric or magnetic fields may also be applied. Through molecular rotation, the dipole moments of individual molecules tend to align with the externally applied field. In Figure 1.10, a rod-like molecule is seen to align with an externally applied magnetic field—this occurrence is termed diamagnetic susceptibility. 


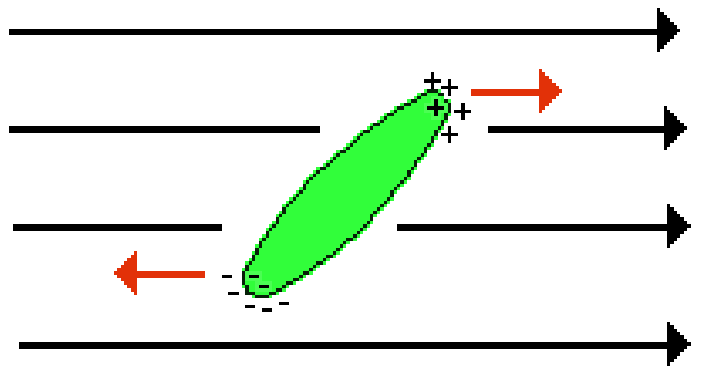

Figure 1.10: The magnetically induced alignment of a polymeric entity. Depending on the strength of the magnetic field, precise degrees of chain alignment may occur. ${ }^{28}$

The morphology of semiconducting polymers within composite films can be tuned by magnetic-field directed assembly, as many research groups have aligned Co-doped $\mathrm{ZnO}$ nanowires with negative paramagnetic anisotropy. ${ }^{29}$ The semiconducting polymer chains, aligned with the $\mathrm{ZnO}$ nanowires, adopt the magnetically induced aligned morphology. However, upon sample rotation, degeneracy is broken and well-aligned mesophases with hexagonal structure are obtained; as a result, routes for the fabrication of nanoporous membranes that can be utilized for sensing and template syntheses have become more robust. ${ }^{29}$ Electric fields have also been routinely used to alter polymer chain morphology. In a study by Lucas et. al., the charge transport properties and hence the alignment of nanocomposite films were altered via an applied electric field. In addition to having enhanced charge mobilities due to the addition of carbon nanotubes (CNTs), P3HT films can realize their optimal charge transport mobilities upon an applied electric field; as such, organic field effect transistors are becoming increasingly viable. ${ }^{30}$ More studies accentuate using an electric field to establish a control of morphology - a macroscopic alignment of polymer nanofibers parallel to the fiber axis was also induced by an electric 
field in one study. ${ }^{31}$ The mechanical and conductive properties of polymers can be greatly enhanced through the increase in anisotropy induced by an applied electric field, and the fabrication of devices utilizing expansive networks of conductive polymers such as biomedical sensors, electrochromic devices, semiconductors, MEMS, and optical devices has become increasingly routine as a result of applied electric field methods. In Figure 1.11, the deformation of a spherical drop of water is observed upon the application of an electric field of increasing magnitude.

a
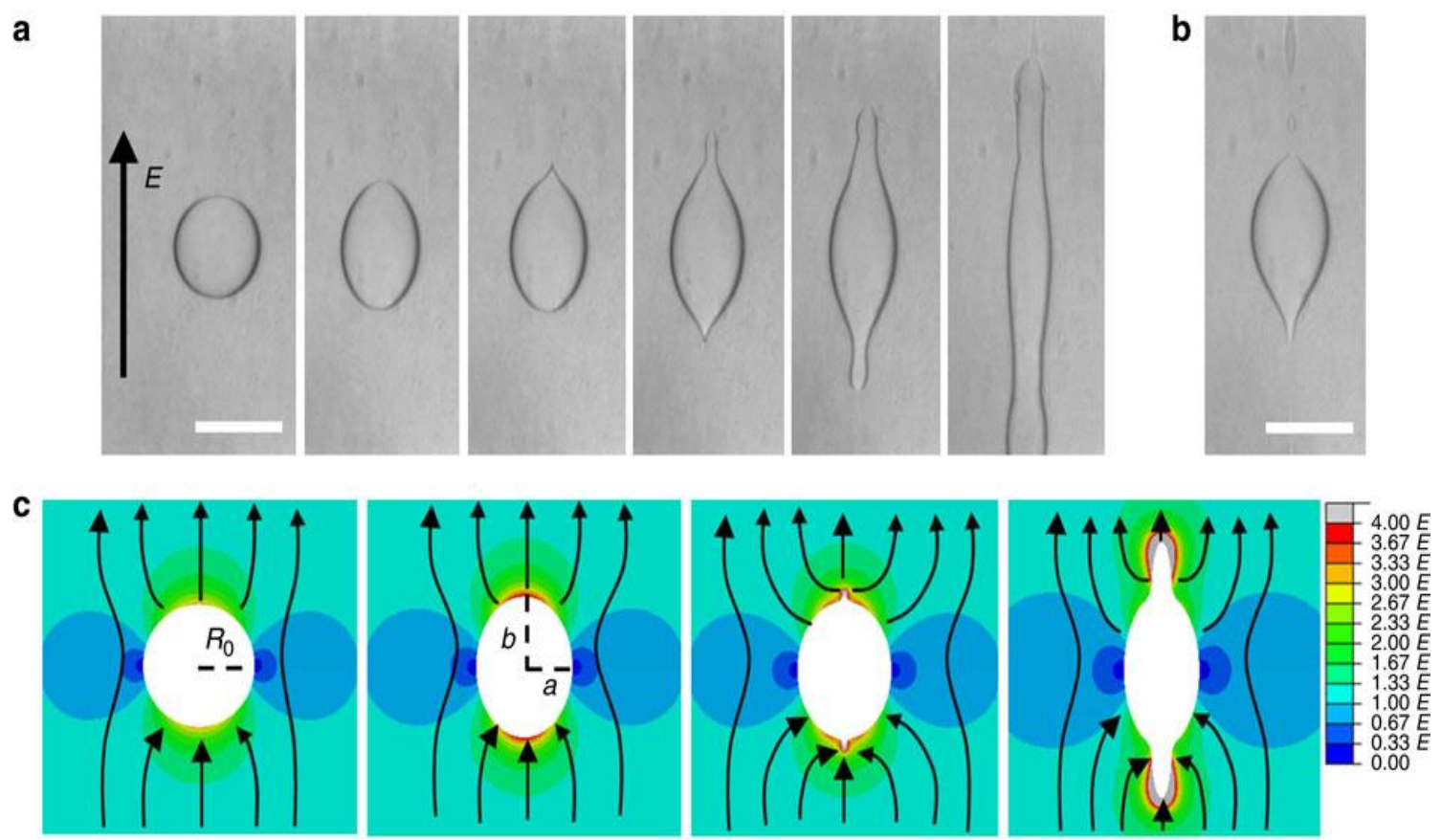

Figure 1.11: Electric Field and Orientation. a) A spherical drop of water deposited on top of a conjugated dielectric polymer layer deforms as the magnitude of an externally applied electric field is increased. b) For most circumstances, there is a memory effect after the removal of an electric field (i.e. the drop cannot deform into its original shape). c) Contours representative of electric field magnitude surround the drop; specific colors denote the intensity of the electric field, as seen in the scale bar to the far right. ${ }^{77}$ 
UV/Vis spectrophotometry is the primary characterization technique for elucidating the morphology of conjugated polymers in solution because it provides indirect evidence of conformational state based on the wavelengths of light absorbed. Information relevant to electronic transitions within atoms can be obtained because upon the absorption of suitable energy from an incident light source, electrons will become excited from a lower energy state to a higher excited state ${ }^{32}$ The extension of conjugation length in a conjugated polymer leads to an enhancement (in terms of absorption intensity), and to a red-shift of the absorption spectrum. ${ }^{32}$ Various methods can be employed to increase the effective conjugation length or intermolecular packing of polymers such as regio-regular P3HT; annealing has been performed reliably to tune chain alignment. In one study, Kang et. al. controlled the orientation of conjugated polymers in the active layer of organic thin-film transistors (OTFTs) by annealing the film at its melting temperature in a vertically aligned multiwalled carbon nanotube (VACNT) template. ${ }^{33}$ Annealing can effectively enhance the crystallinity of a conjugated polymer network, as can the addition of multi-walled carbon nanotubes. In nearly all of these methods utilized to alter the effective conjugation lengths of polymer, UV/Vis spectroscopy is exploited for proof of a change in electronic bandgap magnitude. The 'coil to rod' transition of P3KHT can be probed over time using UV/Vis spectroscopy because the wavelength of maximum absorption of the complex increases as a function of time into the 'red-shifted' region of approximately $600 \mathrm{~nm}$. Before devices are assembled or manufactured on large scale levels, UV/Vis spectroscopy is routinely performed to assess the optoelectronic properties of solution cast thin films, and the morphology is appropriately adjusted based on UV/Vis spectrophotometric results. ${ }^{33}$ 


\subsection{Applications of Conductive Polymers}

As mentioned previously, the discovery of conductive polymers by Heeger et. al. transformed the world of renewable energy and materials science in that cheaper alternative materials for photovoltaic device manufacturing became obtainable. Instead of having to utilize inorganic materials which are typically expensive, environmentally unfriendly, and oftentimes toxic, scientists could rely upon the fact that cheaper organic materials (i.e. conductive polymers) could be employed as electron transport media in photovoltaic devices. Much progress has been made since the initial discovery of conductive polymers by Heeger et. al., as organic photovoltaic devices are being researched around the world by scientists who are striving to engineer the efficiency of the devices to match that of current inorganic solar cells, based on materials such as copper indium gallium selenide (CIGS), or crystalline Silicon. The maximum attainable device efficiency of modern day organic solar cells is inhibited by a series of challenges, one of which is the control of polymeric morphology. If discoveries are made so as to create fully aligned networks of conductive polymers, device efficiencies would likely climb sharply. Herein, specific applications of conductive polymers will be presented, including, but not limited to: organic photovoltaic devices (OPVs), light emitting diodes (LEDs), chemical sensors, and organic field effect transistors (OFETs).

Conductive polymers are perhaps most popularly used for the photoactive layers in organic photovoltaic devices (OPVs). The rapidity of processing OPVs via roll-to-roll or solution based methods is a significant advantage, as more advanced, costly, and time consuming processing steps such as chemical vapor deposition (CVD) are involved in the fabrication of other devices. ${ }^{34}$ In nearly all polymer solar cells, the photoactive layer 
consists of an electron donor and acceptor, wherein conjugated polymers such as polythiophene or polyphenylene serve as typical donor materials. Electron acceptor materials are usually fullerene $\left(\mathrm{C}_{60}\right)$ derivatives and generally possess a lowest unoccupied molecular orbital (LUMO) energy of $0.3-0.4 \mathrm{eV}$ lower than that of the donor. ${ }^{36}$ While the combination of P3HT and the $\mathrm{C}_{60}$ derivative of [6,6]-phenyl-C61butyric acid methyl ester (PCBM) has led to the fabrication of devices with efficiencies beyond 5\%, there are other factors that govern the overall device efficiency, such as molecular weights, polydispersity, regiochemistry, extent of heat treatment, application of electric field, type(s) of solvent added, and the presence of additional additives such as oleic acid. ${ }^{36}$ The molecular structures of either the donor or acceptor may be altered to tune device performance; in one study, instead of simply employing P3HT as a donor, a copolymer consisting of alternating thiophene and cyanovinylene 4-nitrophenyl side segments was employed to enhance the power conversion efficiency (PCE). An increase of $3.7 \%$ in photovoltaic device efficiency was realized upon the introduction of this copolymer as a donor material. ${ }^{36}$ Other details related to molecular structure alter device performance, as an increase in the length of conjugated side chains along the donor material was observed to cause a red-shift of the UV/Vis absorption spectrum. ${ }^{36}$ As changes in the molecular structure of either the donor or acceptor materials can enhance charge transport ability, more research is being conducted to find the optimal blend ratios and molecular structures of both donors and acceptors. Solar cell efficiency and device lifetime are currently the two most significant inhibitors towards mass production, so one of the most popular areas of modern research is in finding improved donor and acceptor materials. 
Because solar cells must exist for years if they are to become profitable for manufacturing, safeguarding the devices against oxidation degradation is essential. Of recent, semiconductor oxides have emerged as replacement materials for PCBM and typically have lower costs - hybrid solar cells with $\mathrm{SnO}_{2}$ instead of PCBM as an electron acceptor have been created. Additionally, electron transfer from $\mathrm{MEH}-\mathrm{PPV}$ to $\mathrm{SnO}_{2}$ was seen to be energetically favorable, occurring on the order of microseconds. A photo induction of charge transfer between $\mathrm{MEH}-\mathrm{PPV}$ and $\mathrm{SnO}_{2}$ can also occur, thus enabling oxides to rival PCBM as alternatives for acceptor materials. ${ }^{38}$ In Figure 1.12, the architecture of a typical polymer solar cell based on bulk heterojunction (BHJ) morphology is depicted.

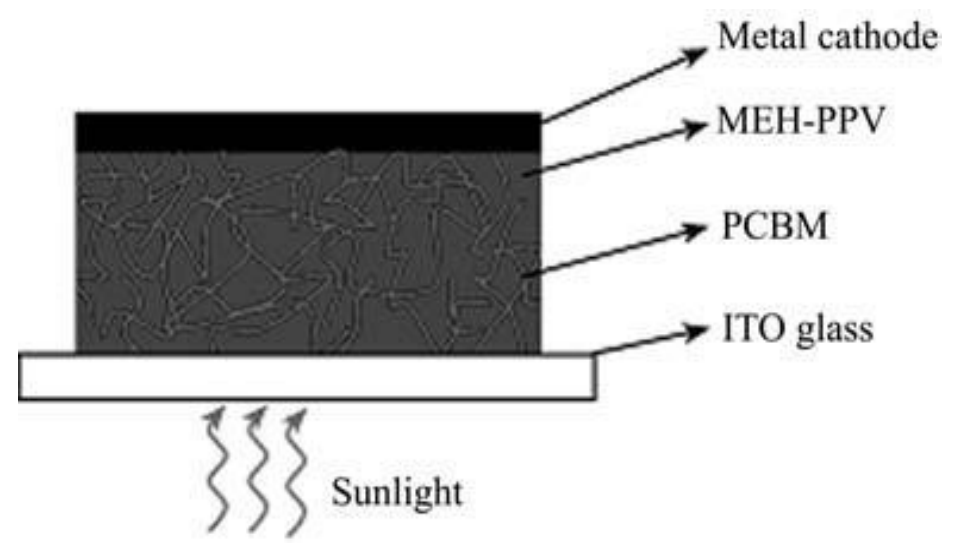

Figure 1.12: Structure of a polymer solar cell with bulk heterojunction (BHJ)

morphology. Sunlight impinges upon the indium tin oxide (ITO) glass anode, electrons are excited from the valence band to the conduction band of the P3HT, excitons are created, and charge transfer occurs across the blended P3HT/PCBM nanodomains. MEHPPV serves as an electron donating material, and once charge is transferred to a metal cathode (typically $\mathrm{Ca}, \mathrm{Al}$, or $\mathrm{Ag}$ ), an electric circuit is completed. ${ }^{64}$ 
Not all organic photovoltaic devices possess bulk heterojunction (BHJ) morphology, as different domain sizes can be obtained through the formation of ordered heterojunction morphologies. In an ordered heterojunction morphology, aligned nanodomains exist and the diffusion length between donor and acceptor is lengthened, thus lessening the possibility that excitons will become annihilated. A downside to an ordered heterojunction morphology is that it is more time consuming and challenging to fabricate; only through the aid of annealing processes or alignment induced processes (i.e. addition of surfactant, nanotubes, or application of a magnetic field) can this optimal morphology be realized. A much larger donor to acceptor interface is created and pathways for charge transfer are much shorter for ordered heterojunction morphologies; as such, attempting to devise this morphology is typically worth the time and resources. An idealized ordered heterojunction morphology should be "like that of two interlocked combs,' and a competition exists between domain purity and size, as smaller domains generally have greater purity. Through polymer self-assembly techniques, methods have been developed to better control the domain size in photovoltaic devices. ${ }^{39}$

In essence, excitons govern the efficiency of a polymer/organic photovoltaic cell, and in order for a device to be successful, excitons must quickly reach the interfaces of donor and acceptor domains before recombination occurs. ${ }^{40}$ Excitons split into free electrons and holes at the donor and acceptor interfaces and must possess lifetimes long enough to be able to reach the device electrodes. Whether or not bulk heterojunction morphologies more easily aid in the generation of electron and hole pairs is still under debate, as Collins and co-workers found that excitons need access to pure aggregates for efficient conversion into charges. Smaller aggregates are deemed better, wherein donor 
and acceptor interfaces exist throughout a larger interfacial area. ${ }^{40}$ Despite the ambiguity in the relationship between donor/acceptor blending methods and device performance, bulk heterojunction solar cells continue to be fabricated regularly in research laboratories. In Figure 1.13, the mechanism behind the generation of an electron/hole pair is depicted.

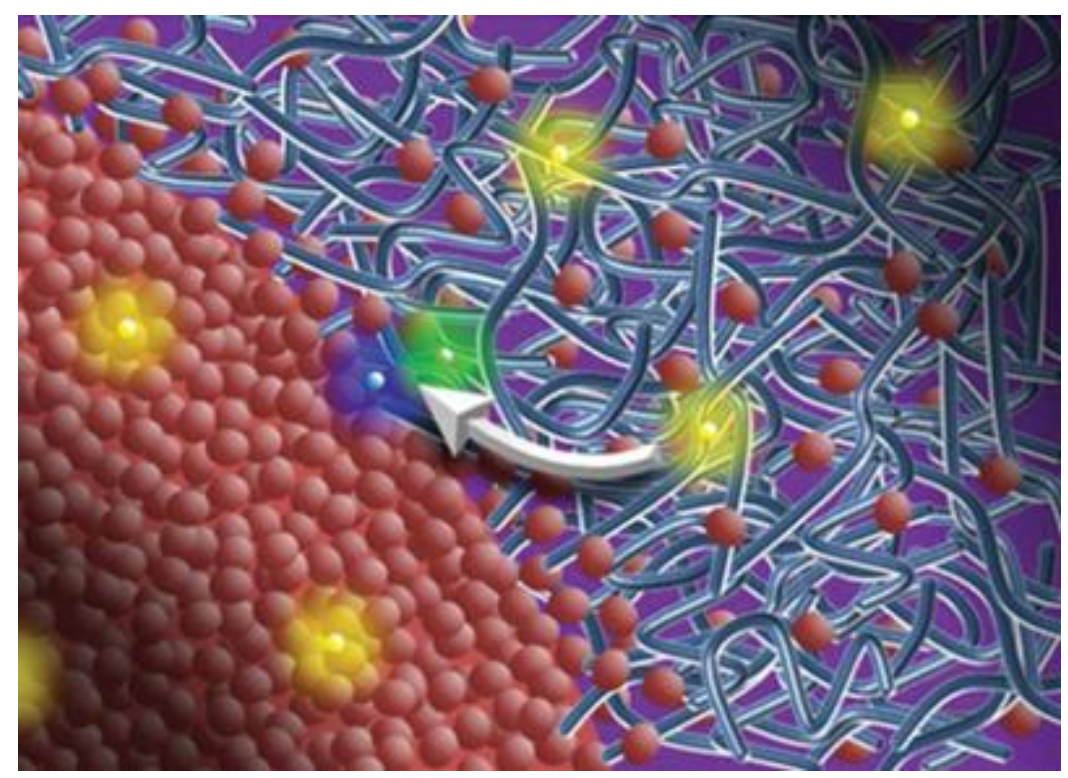

Figure 1.13: Interface between donor and acceptor domains. Red dots represent the acceptor material (organic fullerene molecules), whereas blue lines represent the donor material (conjugated polymer chains). Yellow dots represent excitons, which need to reach the fullerene aggregates to be separated into electrons (purple dots) and holes (green dots). ${ }^{41}$ 


\subsection{Liquid Crystalline Polymers and Their Applications}

While conductive polymers in dilute solution can be utilized for the mass production of organic photovoltaic devices, thin film transistors, and light emitting diodes through drop-casting methods, a wide variety of other devices with different capabilities may be invented through the use of liquid crystalline polymers. When in the liquid crystalline state, polymers exhibit behavior in between that of a solid and liquid; they are considered to be in a fourth state of matter. In polymer/surfactant complexes, the architecture of the surfactant plays a crucial role in determining the onset of liquid crystalline behavior, as the critical concentration, $c^{*}$, above which the complex behaves as a liquid crystal, varies with surfactant type and concentration. Nearly all materials displaying a liquid crystalline nature obey Onsager's rigid rod theory, which states that for sufficiently high concentrations, the alignment of anisotropic particles is favored, despite a decrease in orientational entropy ${ }^{56}$ For concentrations below $c^{*}$, anisotropic particles can freely rotate within solution, assuming a variety of orientations. For most systems, there is no immediate transition from the isotropic (disordered) phase to the liquid crystalline phase, as a transitional or 'biphasic' phase exists, consisting of rods in both the isotropic and liquid crystal phase. Above a certain volume concentration $\Phi_{l}$, the amount of packing entropy increases and the biphasic phase is formed to minimize excluded volume and to maximize free volume. In theory, this phase transition, $\Phi_{1}$, is equal to $3.34(D / L)$, where $D$ is the diameter of a rod particle and $L$ is the length. Thus, for values greater than $3.34(D / L)$, the biphasic phase will exist. Similarly, there exists another critical concentration $\Phi_{2}$, above which the liquid crystalline phase occurs. This secondary critical concentration, $\Phi_{2}$, is equal to $4.49(D / L)$, where $D$ is the diameter of the 
$\operatorname{rod}$ and $L$ is the length. At concentrations above $\Phi_{2}$, the polymer chains will align in parallel with the director $\mathbf{n}$ and the sample will display birefringence when rotated with respect to a polarized light source. The birefringence is due to the sample's dual refractive indices, as light will scatter from the anisotropic particles in one particular direction and intensity when parallel to the director, but will scatter with both different directions and intensities when illuminated at an angle which is not parallel to the director. The sample contrast observed at one angle relative to the light source is typically inverted when the sample is rotated by $45^{\circ}$ relative to this initial angle; regions that originally appeared as red become black, and vice versa. In Figures 1.14 and 1.15, an illustration is provided of the changes in polymer orientation upon phase transitions, as is a pictorial example of birefringence.

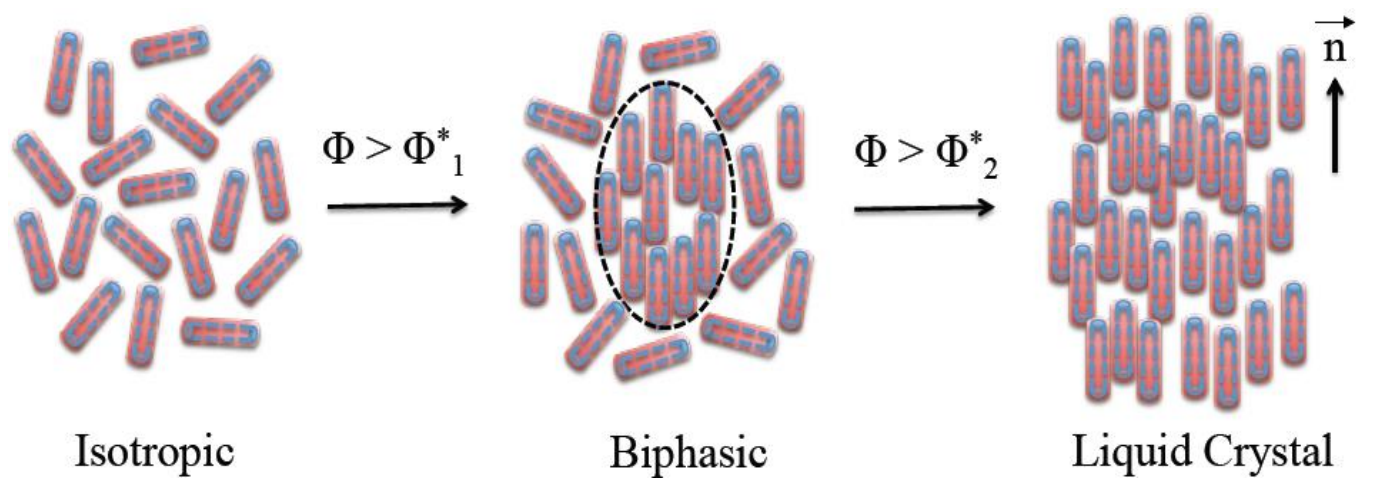

Figure 1.14: Illustration of conjugated polymer conformational changes upon

an increase in concentration. An increase in translational entropy outweighs a decrease in rotational entropy upon an increase in concentration and a parallel alignment of the polymer chains eventually occurs to minimize excluded volume and to maximize free volume, in agreement with Onsager's rigid rod theory. ${ }^{56}$ 

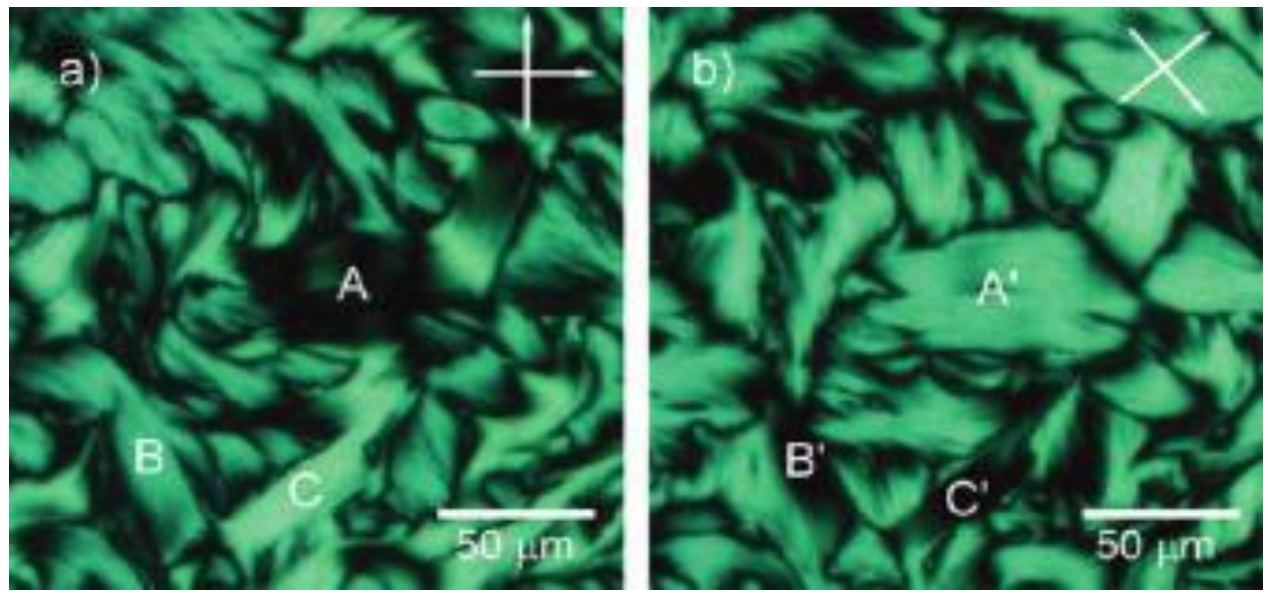

Figure 1.15: Sample birefringence. Dark regions of the sample on the left hand side $\left(0^{\circ}\right)$ become bright upon rotation by $45^{\circ}$, characteristic of anisotropic rigid rods displaying birefringence. ${ }^{80}$

The term 'liquid crystalline' polymer is vague at first glance because many different phases of liquid crystalline polymers may exist. Common liquid crystals consist of a rigid element incorporated into the backbone of normally flexible polymers. ${ }^{44}$ Generally, the rigid element (termed a mesogen) consists of an aromatic group, while the flexible spacer units are of alkyl groups. The aspect ratio, or ratio of length to width or diameter of the rigid element, is a crucial parameter in determining the physical properties of the polymer, as well as which phase(s) the material assumes. Due to a decrease in aspect ratio, the introduction of non-linear cyclic groups on a mesogenic group will lower the temperature at which the material transitions into the isotropic phase. ${ }^{74}$ The different phases which liquid crystalline polymers may form are termed 'mesophases,' and the simplest of these phases is the nematic phase. The nematic phase possesses the lowest ordering of all mesophases, wherein the axes of the mesogens are 
aligned in parallel, but may nevertheless move with respect to one another like in the liquid phase. All movements are achieved on the same plane in the nematic phase and there is no common direction as a whole. Individual planes of liquid crystals that are vertically stacked along an imaginary axis orthogonal to the director will likely not exist in the nematic phase. ${ }^{45}$ A liquid crystalline phase harder to obtain is the smectic phase, which is more representative of the solid phase. The long axes of the mesogens align parallel with each other in that individual layers are stacked in unison. The polymer rods orientate in the same general direction and exhibit a much higher degree of order as compared to the nematic phase; the smectic phase is the last mesophase to occur before the polymer reaches the solid phase. ${ }^{46}$ In Figure 1.16 , the transitions of liquid crystal mesophases are shown with respect to increasing temperature.

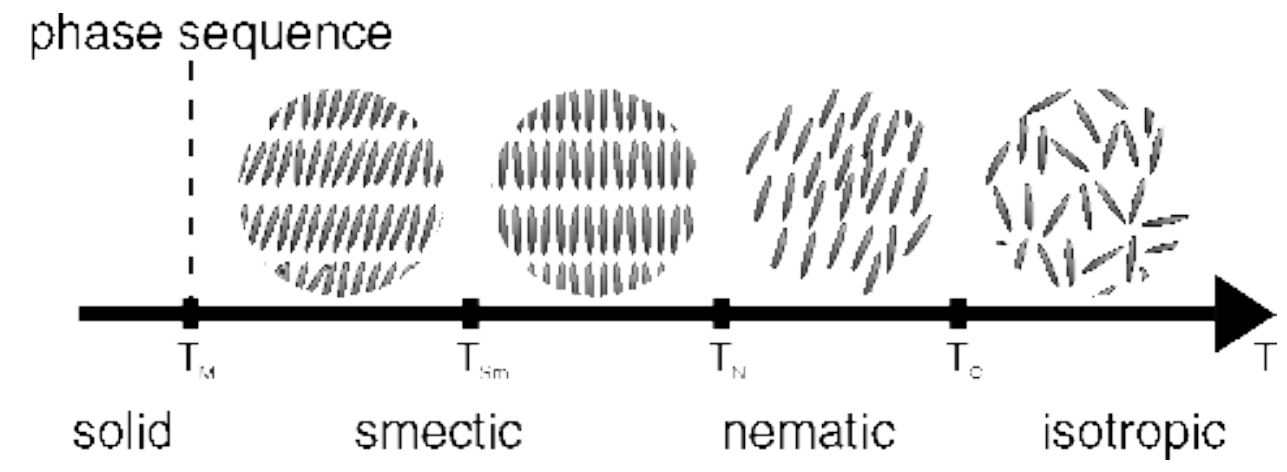

Figure 1.16: Phase sequence of liquid crystalline polymers for increasing

temperature. There are critical temperatures associated with the formation of each phase, labeled along the temperature axis. It should be noted that the liquid crystals depicted above are thermotropic in that they change phases with respect to temperature. Lyotropic liquid crystals change phase with respect to concentration. ${ }^{65}$ 
Now that the general properties and principles governing liquid crystal ordering have been discussed, it is necessary to comment on some of the most frequent applications of liquid crystalline polymers. Of recent, the prices of liquid crystalline polymers have fallen substantially, and as such, the market has been able to benefit considerably. Because liquid crystalline polymers are mechanically robust and offer heat resistance, they can oftentimes replace metals for automotive, electronic, and space technology applications. ${ }^{48}$ Liquid crystalline polymers are contained in specialized clothing for sports, firefighting, or chemical protection; in Table 2, the most common applications of liquid crystalline polymers are mentioned. One should note the many high strength applications - because liquid crystalline polymers are oftentimes organically based, they can be prepared via low cost methods and offer much cheaper alternatives to high strength materials or apparel based on metals or various alloys.

Table 2: Various Applications of Liquid Crystalline Polymers. ${ }^{44}$

\begin{tabular}{|c|c|c|}
\hline End Use & End Use Systems & Key Attributes \\
\hline Composites & $\begin{array}{l}\text { Fabrics for aircraft } \\
\text { and containers, } \\
\text { pressure vessels, } \\
\text { ship building, } \\
\text { sporting goods, } \\
\text { plastics additives, } \\
\text { structures for civil } \\
\text { engineering. }\end{array}$ & $\begin{array}{ll}\text { - } & \text { Light weight. } \\
\text { - } & \text { High strength. } \\
\text { - } & \text { High modulus. } \\
\text { - } & \text { Good impact } \\
\text { - } & \text { strength. } \\
\text { - Wear resistance. }\end{array}$ \\
\hline
\end{tabular}


Table 2, Continued

\begin{tabular}{|c|c|c|}
\hline Protective Apparels & $\begin{array}{l}\text { Heat resistance and } \\
\text { work wear, fire } \\
\text { blankets, flame } \\
\text { retardant textiles, } \\
\text { cut protective } \\
\text { gloves, cut } \\
\text { protective seat } \\
\text { cover layers. }\end{array}$ & $\begin{array}{l}\text { - Heat resistance. } \\
\text { - } \quad \text { Cutame retardation. } \\
\text { - }\end{array}$ \\
\hline Tires & $\begin{array}{l}\text { Truck and aircraft } \\
\text { tires, high speed } \\
\text { tires, motorcycle } \\
\text { tires, bicycle tires. }\end{array}$ & $\begin{array}{l}\text { - Low density. } \\
\text { - Weight saving. } \\
\text { - } \quad \text { Low shrinkage. }\end{array}$ \\
\hline Mechanical Rubber Goods & $\begin{array}{l}\text { Conveyor belts, } \\
\text { transmission belts, } \\
\text { hoses for } \\
\text { automotive, } \\
\text { hydraulic hoses, } \\
\text { hoses in off-shore } \\
\text { umbilical. }\end{array}$ & $\begin{array}{ll}\text { - } & \text { High strength. } \\
\text { - } & \text { Dimensional } \\
\text { stability. } \\
\text { - } \text { Thermal resistance. } \\
\text { - } \text { Chemical } \\
\text { resistance. }\end{array}$ \\
\hline $\begin{array}{l}\text { Friction Products and } \\
\text { Gaskets }\end{array}$ & $\begin{array}{l}\text { Brake linings, } \\
\text { clutch facings, } \\
\text { gaskets, thixotropic } \\
\text { additives, industrial } \\
\text { paper. }\end{array}$ & $\begin{array}{ll}\text { - } & \text { Heat resistance. } \\
\text { - } & \text { Chemical } \\
& \text { resistance. } \\
\text { - } & \text { Low flammability. } \\
\text { - } & \text { Mechanical } \\
& \text { performance. } \\
\end{array}$ \\
\hline Ropes and Cables & $\begin{array}{l}\text { Aerial optical fiber } \\
\text { cable, traditional } \\
\text { optical fiber cable, } \\
\text { electrocable. }\end{array}$ & $\begin{array}{ll}\text { - } & \text { High strength. } \\
\text { - } & \text { High modulus. } \\
\text { - } & \text { Dimensional } \\
& \text { stability. }\end{array}$ \\
\hline Life Protection & $\begin{array}{l}\text { Bullet proof vests, } \\
\text { helmets, property } \\
\text { protection panels, } \\
\text { vehicle protection, } \\
\text { strategic equipment } \\
\text { shielding. }\end{array}$ & $\begin{array}{l}\text { - High tenacity. } \\
\text { - High energy } \\
\text { dissipation. } \\
\text { - Low density and } \\
\text { weight reduction. } \\
\text { - } \quad \text { Comfort. }\end{array}$ \\
\hline
\end{tabular}

Liquid crystal polymers can be mechanically enhanced by adding different fibers to create a composite structure. Protective clothing listed in Table 2, such as bullet proof 
vests or heat resistant clothing, is manufactured by combining aligned liquid crystalline polymers with high strength fibers. The heat resistant pants and jackets which firefighters wear are composed of poly-m-phenyleneisophthalamide, or Nomex, an aramid fiber with excellent thermal stabilities. ${ }^{44}$ In addition to the ability of liquid crystalline polymers to mechanically enhance products such as tires, fabrics for aircraft, brake linings, or optical fiber cables, the manufacturing of printed circuit boards and other electronic devices has escalated as a result of liquid crystalline polymer versatility. In a study by Farrell et. al., liquid crystal polymers were seen to offer numerous advantages in advanced printed wiring board and packaging applications. Due to their low permeability, high temperature durability, low dielectric constant, low moisture absorption, and smooth surfaces, liquid crystal polymers have served as excellent surface mounts for printed wiring boards and optoelectronic applications. ${ }^{46}$ The extraordinary barrier properties that liquid crystals offer make them suitable for integration into high powered circuits, where impermeability to moisture and various gases is critical to ensure optimal performance. Liquid crystals were found to have satisfied all of the requirements for a base laminate in a thin film circuit, such as resistance to wet etch chemistries and strong adhesion to electrodeposited copper layers. ${ }^{47} \mathrm{~A}$ finish metallization such as tin or lead solder must be applied to a printed circuit in order to protect an electrodeposited copper conductive layer from corrosion; these metallization processes generally require extremely high temperatures and can contaminate underlying layers. Liquid crystal polymer protective substrates were seen to be as robust as more expensive inorganic or transition metal protective substrates in protecting the underlying circuit from contamination, etc. Based on the findings of Farrell et. al., it is without a doubt that near-hermetic cavity packages for printed circuits 
and MEMS devices can be made successfully and at low costs via the use of liquid crystalline polymeric substrates. The aforementioned applications of liquid crystalline polymers are only a few of many continually emerging uses.

Liquid crystalline polymers cannot realize their full potential as charge transport mediums, components in waveguides, or as high strength materials unless a satisfactory alignment is obtained of the liquid crystals. The most common techniques available to align liquid crystals are the application of electric and or magnetic fields, and the application mechanical force (i.e. shear flow). In many studies, a constant shear rate is used in a temperature range around the isotropic to nematic transition temperature. The molecular weight of the polymer also plays a role in how easy it may be to align liquid crystals via shear force, as higher molecular weight samples exhibited alignment only at lower flow alignment angles. ${ }^{78}$ The application of unidirectional shear forces has been observed to induce alignment of conjugated polymer chains upon solvent evaporation. ${ }^{79}$ Moreover, upon the application of force, alignment of the chains parallel to the shear direction occurred two out of three times and alignment perpendicular to the shear direction occurred one out of three times based on a study by Bae et. al. ${ }^{79}$ Analysis of absorbance and photoluminescence spectra is commonly conducted in order to verify the morphological changes believed to occur following the application of shear flow. 


\section{Experimental Methods}

\subsection{Material Preparation}

An assortment of surfactants were used in conjunction with a conjugated polyelectrolyte to facilitate production of hydrogel complexes. Regioregular (82-90\% head-to-tail) poly[3-(potassium-6-hexanoate)thiophene-2,5-diyl] (P3KHT, weight average molecular weight $M_{w}=60 \mathrm{~kg} / \mathrm{mol}$, Rieke Metals, Inc.), cetyl trimethylammonium bromide (CTAB 'C16,' Sigma Aldrich), Myristyltrimethylammonium bromide ('C14,' Sigma Aldrich), Dodecyltrimethylammonium bromide ('C12,' Sigma Aldrich), Decyltrimethylammonium bromide ('C10,' Sigma Aldrich), and dihexadecyl dimethyl ammonium bromide (DHAB, Sigma Aldrich) were used as received in order to prepare the supramolecular complexes. The P3KHT varied in terms of both appearance and behavior from batch to batch; some consignments consisted of a finely ground black powder, whereas others consisted of hard chunks of P3KHT that did not dissolve immediately in water. The time dependent chromism was affected by the physical properties of the P3KHT because the variation in molecular weight distribution from batch to batch influenced the kinetics of the dynamic chain unfolding process. All surfactants appeared as white powders; CTAB as a moderately ground power, 'C14' as an extremely finely ground powder, 'C12' as a powder with salt-like particulates, and 'C10' as a moderately ground powder. DHAB appeared as a thick and sticky powder which was hard to extract from the chemical bottle - a fine spatula had to be used for its removal. The molecular structures of CTAB, DHAB, and P3KHT are displayed in Figure 2.1: 


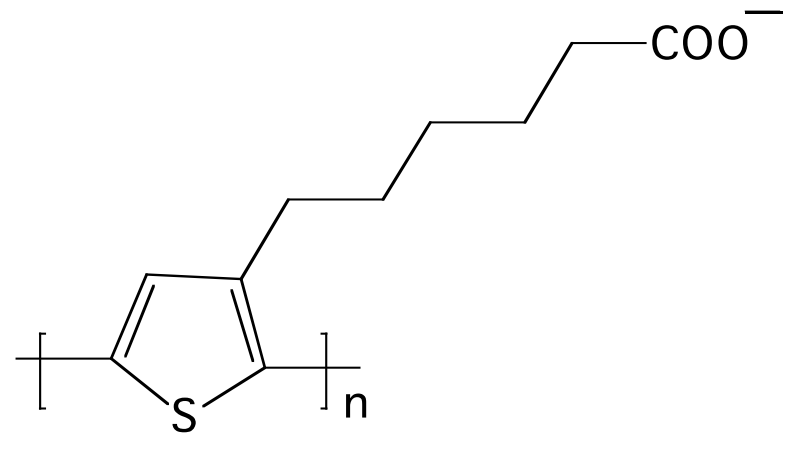

(a)

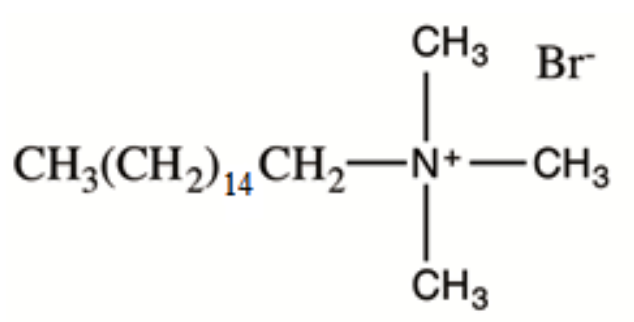

(b)

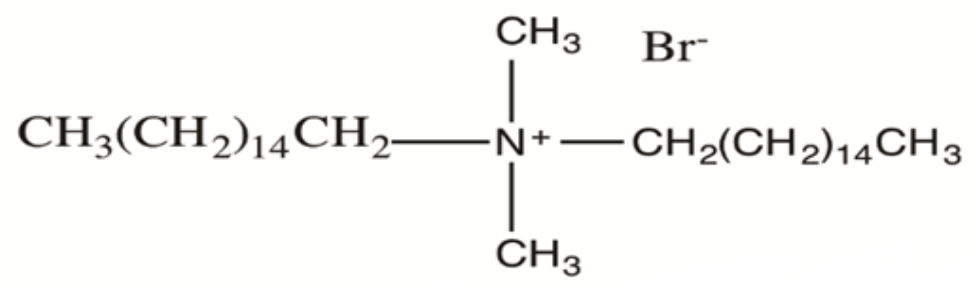

(c)

Figure 2.1: Molecular Structures. (a) P3KHT, (b) CTAB, and (c)

DHAB.

In order to prepare hydrogels, specific amounts of polymer and surfactant were added so as to yield a complex of $0.10 \mathrm{M}$ concentration. Firstly, P3KHT $(0.0234 \mathrm{~g}$, $0.0001 \mathrm{~mol}$ ) was dissolved in $0.5 \mathrm{~mL}$ of $18 \mathrm{M} \cdot \Omega$ Milli-Q Nanopure water in a single plastic vial. Various weights of surfactant were added to another vial separately to produce a $0.20 \mathrm{M}$ solution in $0.5 \mathrm{~mL}$ of $18 \mathrm{M} \cdot \Omega$ Milli-Q Nanopure water; these solutions were subsequently oven heated at $50^{\circ} \mathrm{C}$ for at least 30 minutes to aid in the dissolving of the surfactant. For all pure surfactant solutions, $0.0001 \mathrm{~mol}$ of a specific surfactant was added to $0.5 \mathrm{~mL}$ water, corresponding to $0.028029 \mathrm{~g}$ 'C10,' $0.030834 \mathrm{~g}$ 'C12,' $0.033639 \mathrm{~g}$ 'C 14 ,' and $0.036445 \mathrm{~g} \mathrm{CTAB}$ 'C16,' respectively. The amount of DHAB added never exceeded $30 \%$ of the total molar surfactant concentration - for instance, a 30\% DHAB solution was 
prepared by weighing $0.30(0.0001 \mathrm{~mol})=0.00003 \mathrm{~mol} \mathrm{DHAB}(0.01724 \mathrm{~g})$ and $0.70(0.0001)=0.00007 \mathrm{~mol} \mathrm{CTAB}(0.0255 \mathrm{~g})$, followed by dissolving these amounts in $0.5 \mathrm{~mL}$ of nanopure water. $\mathrm{DHAB}$ took a considerably long time to fully dissolve in water, and as such, DHAB containing solutions were oven heated for at least 90 minutes at a temperature greater than $50^{\circ} \mathrm{C}$. Once the polymers and surfactants were fully dissolved in their respective solutions, surfactant was added to the polymer solution dropwise via a micropipette. After all surfactant was added to the polymer solution (thereby rendering a $0.10 \mathrm{M}$ solution in $1 \mathrm{~mL}$ of total water), the vial was centrifuged at 10,000rpm (18-Centrifuge Biotechnical Services, Inc.) for at least 30 minutes to create a separation between solid phases and supernatant. Over time, the viscosity of the hydrogel increased substantially, wherein a thick gel resulted. Viscosity increased in direct proportion to DHAB content and distinct phase separation of the surfactant from the supernatant was observed for solutions containing 20 or $30 \%$ DHAB after centrifugation.

All dilute complex solutions used for either UV/Vis spectroscopy or fluorescence were obtained by extracting a small amount of the supernatant phase with a micropipette and adding this amount dropwise to more nanopure water. Solutions of high concentration utilized for nematic liquid crystal studies were prepared by completely evaporating the water from a hydrogel solution and then recording the total solid weight of a hydrogel (i.e. the total weight of polymer and surfactant content). A designated amount of water was then added to the dried complex in order to yield a solution of the desired weight percent concentration. For instance, if the combined dry weight of polymer and surfactant was $2 \mathrm{~g}$, approximately $0.8571 \mathrm{~g}(0.8571 \mathrm{~mL})$ of nanopure water was added to render the concentration $70 \mathrm{wt} . \%\left[(2 \mathrm{~g}\right.$ solid $) /\left(2 \mathrm{~g}\right.$ solid $\left.\left.+0.8571 \mathrm{~g} \mathrm{H}_{2} \mathrm{O}\right)\right]=$ 
$0.70=70 \%$. All high concentration solutions had to be oven heated for at least two hours at $50^{\circ} \mathrm{C}$ or above to aid in homogenization. It was imperative to obtain homogeneous solutions, as any inhomogeneity would be likely to induce erroneous results in experimentation due to discrepancies in concentration, and hence in the identity of the phases. The accuracy of the solution concentrations was tested via a 'drop/dry' test, wherein the weight of a weigh boat and small glass cover slide was recorded. A small drop of the solution to be assessed was deposited onto the glass cover slide and the weight of the weigh boat, cover slide, and drop was recorded. The drop was allowed to dry overnight in an oven, and the weight of the weigh boat, cover slide, and dried drop was recorded. The experimentally determined concentration of the solution in question was thus determined via equation 1:

Concentration $($ Wt. \% $)=\left(W_{\text {dried drop }}(\mathrm{g})-\mu(\mathrm{g})\right) /\left(W_{\text {wet drop }}(\mathrm{g})-\mu(\mathrm{g})\right)$

where $\mu$ refers to the combined weight in grams of the weigh boat and glass cover slide, $W_{\text {dried drop }}$ is the weight of the dried drop in grams, and $W_{\text {wet drop }}$ is the weight of the wet drop in grams.

The solid state complexes were prepared by depositing a small amount of stock hydrogel on a glass slide and smoothing out the gel by pressing down with a cover slide. The glass slide with the deposited gel was oven heated for at least six hours so that a completely dry film was obtained. Although no quantitative tests were conducted to prove that the resulting film was dry, intuition led to the determination of dryness; if the film was capable of being 'chipped' with a razor blade, it was deemed dry. 


\subsection{Instrumental Methods of Analysis}

\subsubsection{UV/Vis Spectrophotometry}

A Jasco V-550 spectrophotometer was utilized for obtaining UV/Vis absorbance data. Standard plastic stirring cuvettes were employed for the analysis and complete homogenization of the diluted solutions was verified by stirring the contents of a cuvette with a pipette tip. The cuvette to be analyzed was placed in the first slot of the spectrophotometer and the top cover of the instrument was closed to protect the sample from the ambient atmosphere. A 'blank' sample was first recorded by running a UV/Vis spectrum on a cuvette containing nanopure water. The spectrum obtained from this 'blank' solution was recorded and referenced as a baseline for subsequent measurements. To enhance the validity of time dependent data (i.e. absorbance corresponding to a specific time after dilution), a predetermined amount of stock hydrogel was added to a cuvette already containing nanopure water. ' $t=0$ ' was defined as the moment in which the stock hydrogel was added to the cuvette via a micropipette. It should be noted that all solution concentrations for UV/Vis spectroscopy were $0.133 \mathrm{mM}$; in order to prepare this concentration, $50 \mu \mathrm{L}$ of the top phase supernatant was added to $833 \mu \mathrm{L} \mathrm{H}_{2} \mathrm{O}$ to render a $6 \mathrm{mM}$ solution concentration. This 'precursor' solution was made so that a reasonably large volume of solution could be extracted during the final dilution to mitigate uncertainty in volume. Subsequently, $40 \mu \mathrm{L}$ was extracted from the $6 \mathrm{mM}$ solution and dissolved in $1.8 \mathrm{~mL} \mathrm{H}_{2} \mathrm{O}$ to render a final concentration of $0.133 \mathrm{mM}$. 


\subsubsection{Fluorometry}

A Jasco FP-6500 spectrofluorometer was utilized for all fluorometry studies; the excitation wavelength was set to $430 \mathrm{~nm}$ and the emission wavelengths were dependent on the solution composition (i.e. surfactant architecture). An expected emission wavelength of 550nm was entered into the Jasco software. Specialized cuvettes which were transparent on all four sides were used for fluorescence and both shutters were opened for operation to allow for the light source to fully illuminate the sample. The cover of the instrument was fully closed prior to performing experiments to protect the sample from the surrounding environment as well as from additional light sources. All cuvettes were cleaned thoroughly with Liquinox detergent solution and Deionized water on a regular basis to eliminate the buildup of contaminants.

\subsubsection{Dynamic Light Scattering (DLS)}

\section{A Wyatt Technologies DynaPro Nanostar Dynamic Light Scattering (DLS)} instrument was utilized to acquire information related to the average particle size within a polymer/surfactant complex. The wavelength of the DLS instrument was fixed at $633 \mathrm{~nm}$ and a He-Ne laser source produced the incident light. All samples to be analyzed were first diluted to the desired concentration of $13.3 \mu \mathrm{M}$ and were introduced into a small plastic cuvette, which was subsequently placed inside of the instrument. Upon closing the shutter to the instrument, a spectrum was acquired wherein the average particle size of the solution was determined, along with the standard deviation. All averages were based on ten (10) acquired data points and the instrument was set to 'Random Coil' mode. Cuvettes were thoroughly cleaned in between sample measurements with Liquinox detergent and deionized water. 
The DLS instrument translates the intensity of scattered light into information regarding macromolecular size distributions. After light bombards the molecules in the sample, the light is diffracted and either interferes constructively or destructively; the scattered light enters a photomultiplier and an image is projected onto a phosphor screen. Typically, a 'speckle' pattern is produced and the intensity of light spots is analyzed over time. The intensity and position of the light spots on the phosphor screen are related to both the Brownian motion of the particles as well as to their size distribution profile. The mean effective diameter of particles determined from DLS is dependent upon the particle core size, surface geometry, particle concentration, and type of ions in the medium. ${ }^{68}$

\subsubsection{Polarized Optical Microscopy (POM)}

A Leica DM2500P Polarized Optical Microscope was utilized for observing the liquid crystal characteristics of high concentration complexes. Through a contrast enhancing technique (the blocking of one component of the transmitted light via a polarizer oriented at 90 degrees to the illumination source), a polarized light source can interact strongly with a sample to provide information related to a sample's optical path boundaries. Different indices of refraction within a particular sample will lead to the occurrence of birefringence, wherein varying levels of color contrast will occur in direct proportion to refractive index dissimilarities. The variations in refractive index across the sample occur due to anisotropic features which block certain polarization angles of light. Thus, POM is a viable technique for elucidating information specific to a sample's structure and composition. In POM, the sample is fixed to a 360 degree rotating specimen stage and is observed through a conventional microscope eyepiece. Light from the bottom of the microscope stage becomes polarized after passing through a grating and is 
directed towards the sample — unless the sample is birefringent, the light source will not be repolarized to the angle which it assumed prior to entering the grating. There will be no transmission of light if the sample is not birefringent (i.e. isotropic), and thus one can deduce structural information of the sample by correlating well-ordered and anisotropic polymer orientations to birefringence, and disordered orientations to an isotropic phase.

On the Leica DM 2500P, the available magnification levels are 5X, 10X, 20X, 30X, and 40X, respectively. Once a particular magnification was selected, the image was focused and the sample was rotated by 90 degrees. Images were taken of all samples at orientations of 0,45 , and 90 degrees, respectively, and the levels of birefringence were analyzed. In general, birefringence was denoted by areas of inverse contrast (i.e. black areas turning red or vice versa) upon rotation of the sample by 45 degrees. All samples were prepared by depositing a concentrated solution of the desired complex onto a glass slide and by spreading the solution through applying pressure to a small glass cover slide above the sample until a non-transparent and homogeneous thin film was observed. It should be noted that the accepted errors were considered to be $\pm 2 \mathrm{wt} . \%$ for all sample concentrations studied under POM. The justification for this level of error stems from the fact that the concentrations determined experimentally from the 'drop/dry' test were on average within $\pm 2 \mathrm{wt}$. $\%$ of the theoretically predicted concentrations. No errors are reported with respect to the molar percent of DHAB within a complex, as any errors in the CTAB: DHAB ratio were considered negligible. 


\subsubsection{Differential Scanning Calorimetry (DSC)}

The melting behaviors and crystalline natures of solid state complexes were assessed via differential scanning calorimetry. Regular stock hydrogel solutions of $0.10 \mathrm{M}$ concentration were deposited onto glass slides and dried overnight in an oven at a temperature greater than or equal to $50^{\circ} \mathrm{C}$ to produce thin solid films. Small chunks of the dried films were used as samples for DSC; a spatula was used to chip off the chunks and the chunks were subsequently weighed along with a standard DSC pan and lid. Enough of the solid sample was added to exceed $5 \mathrm{mg}$ of total weight, as $5 \mathrm{mg}$ is the minimum permissible sample weight for DSC. After recording the respective weights of the sample, pan, and lid, the sample was sealed into the pan by pressing and sealing the pan against the lid through use of a 'crimper.' Samples were loaded into a TA Instruments DSC Q1000 and were heated at $5^{\circ} \mathrm{C} / \mathrm{Min}$. from $50^{\circ} \mathrm{C}$ to $120^{\circ} \mathrm{C}$. Three temperature sweeps were performed: a preliminary heating cycle, a cooling cycle, and a final heating cycle. The pertinent thermal information was determined from the final heating cycle. All phase transitions (i.e. $T_{g}, T_{c}$, and $T_{m}$ ) were determined by noticing either first or second order thermal transitions. TA Instruments software was utilized to interpolate the thermal transitions via approximations of local maxima. The enthalpy of fusion was found by integrating the heat flow curve from the onset and offset of the respective transition.

\subsubsection{X-Ray Diffraction (XRD)}

X-Ray Diffraction was used to gain a better understanding of the atomic and molecular structure within the sample(s) of interest. The instrument used was a reflection

geometry Siemens D5000 with a $1.54 \AA \mathrm{Cu} \mathrm{K} \alpha$ radiation source; all spectra were obtained 
via a source current of $5 \mathrm{~mA}$ and an accelerating voltage of $40 \mathrm{kV}$. A scan rate of one

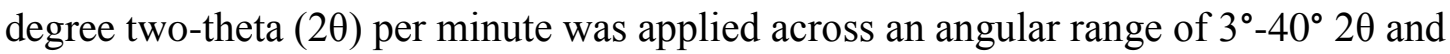
spectra were saved in text format to be analyzed via Excel software. Powder samples were compressed in a specialized powder sample holder with a flat aluminum tray, and the sample holder was loaded into the instrument by securing it with three prongs to a flat substrate. Thin film (non-powder) samples were ensured for homogeneity and were dried onto a glass cover slide overnight at a temperature greater than $50^{\circ} \mathrm{C}$. The cover slide was then inserted into the X-Ray diffractometer and was secured to a flat substrate via a three prong attachment. Background subtraction was not performed in any of the obtained XRD spectra because it was assumed that the glass or plastic substrate did not exhibit any diffraction peaks in the angular region of interest. If there was any diffraction resulting from the background substrate, it would have resulted in peaks of extremely low intensity due to the homogeneity of the thin film sample. The deposited and dried samples rendered the glass substrate opaque in appearance (i.e. no part of the glass substrate was visible or exposed to the X-Ray source), justifying the assumption that no background spectra would appear.

\subsubsection{Small Angle X-Ray Scattering (SAXS)}

Attempts were made to perform Small Angle X-Ray Scattering (SAXS) to elucidate polymer conformation in high concentration (liquid crystalline) solutions. A pin-hole collimated Rigaku instrument (SMAX 3000) with a $1.54 \AA$ Cu Ka radiation source was used on all samples. The SAXS instrument was located in the lab of Dr. Chinedum Osuji, a Professor of Chemical Engineering at Yale University in New Haven, CT. SAXS is a technique almost entirely specialized for polymer analysis. Detailed 
studies of polymer morphology, orientation, and structure are acquired through SAXS, and it is currently the most favorable technique for obtaining high resolution structural information of chain entanglements and orientation, etc. ${ }^{82}$ The general theory behind the operation of SAXS is that a high power X-Ray source (i.e. $\mathrm{Cu} \mathrm{K} \alpha$ ) is placed through a monochromator, mirror apertures, and a shutter under ultra-high vacuum conditions. After the beam is monochromated, it is less capable of inducing damage, as the beam energy is reduced along with the spot size. Upon interacting with the polymer sample, the X-Ray beam scatters in a specific pattern characteristic of the material; the scattering of the X-Ray beam is recorded on a photographic plate located a few millimeters behind the sample. Certain polymer morphologies exhibit differing scattering patterns. Highly crystalline samples yield nearly circular patterns, whereas amorphous samples (i.e. quartz polymer) tend to yield broad and less sharp scattering patterns. ${ }^{83}$ In Figure 2.2, an assortment of scattering patterns are presented that correspond to sample characteristics.

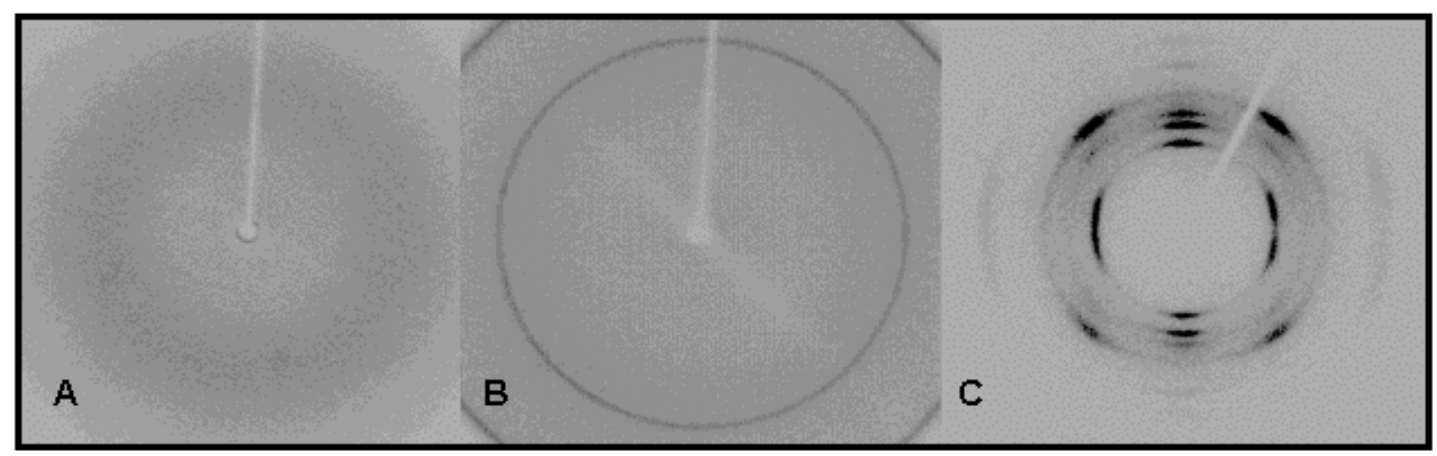

Figure 2.2: SAXS patterns. Sample A: Amorphous material. Sample B:

Polycrystalline material. C. Oriented Polycrystalline and Amorphous material. ${ }^{48}$

The most conventional method of interpreting SAXS data is through an intensity versus scattering vector plot or through a Guinier Plot. A traditional plot records the 
intensity of scattered X-Rays on the $y$-axis and the scattering vector $q$ on the $x$ axis. The scattering vector $q$ is related to the elastic interaction between the X-Ray beam and the sample and is defined:

$q=4 \pi \sin (\theta) / \lambda$

where $\theta$ is the scattering angle in degrees and $\lambda$ is the X-Ray wavelength in $\mathrm{nm}$. The scattered X-Ray intensity, $I(q)$, is related to the electron density function $R(r)$. Thus, regions with greater electron density will scatter X-Rays to a larger extent, and through a Fourier Transform of the electron density correlation function $g(r)$, X-Ray scattering patterns will reveal the spatial correlations in a sample. ${ }^{48}$ As seen in Figure 2.3, a conventional SAXS setup consists of an X-Ray source, a monochromator, a sample holder, and a background detector:

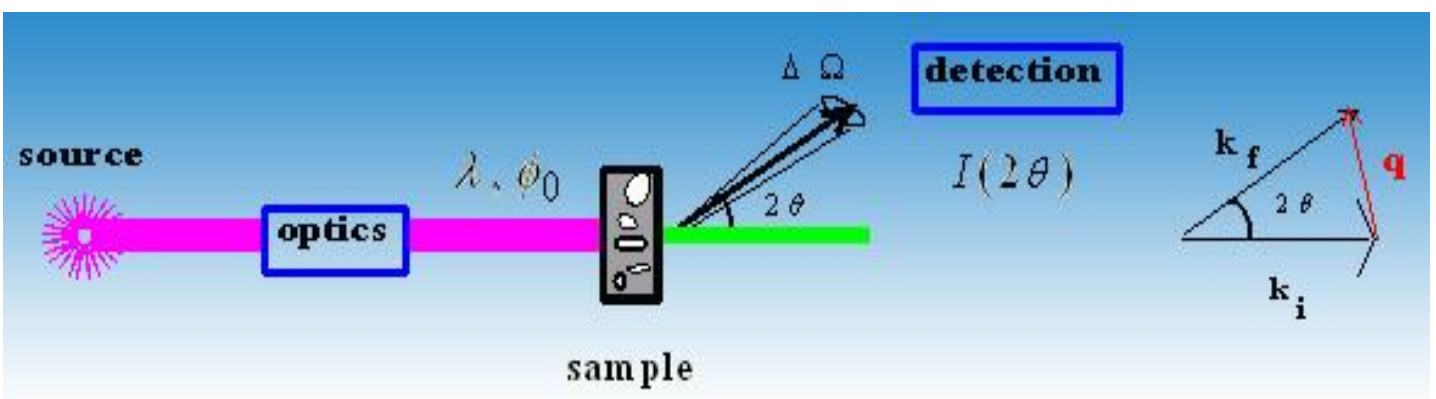

Figure 2.3: A conventional SAXS instrumental setup. ${ }^{48}$

From the previously discussed plot of scattered intensity $I(q)$ versus scattering vector $q$, the most valuable piece of information that a polymer scientist can determine is the Radius of Gyration, $R_{g}$. As seen in Figure 2.4, the slope of a graph of $\ln (I(q))$ versus $q^{2}$ can be linearly approximated and set equal to $R_{g}$. This type of plot is referred to as a 
Guinier Plot after the French physicist Andre Guinier who discovered the relationship of particle size to scattered X-Ray intensity.

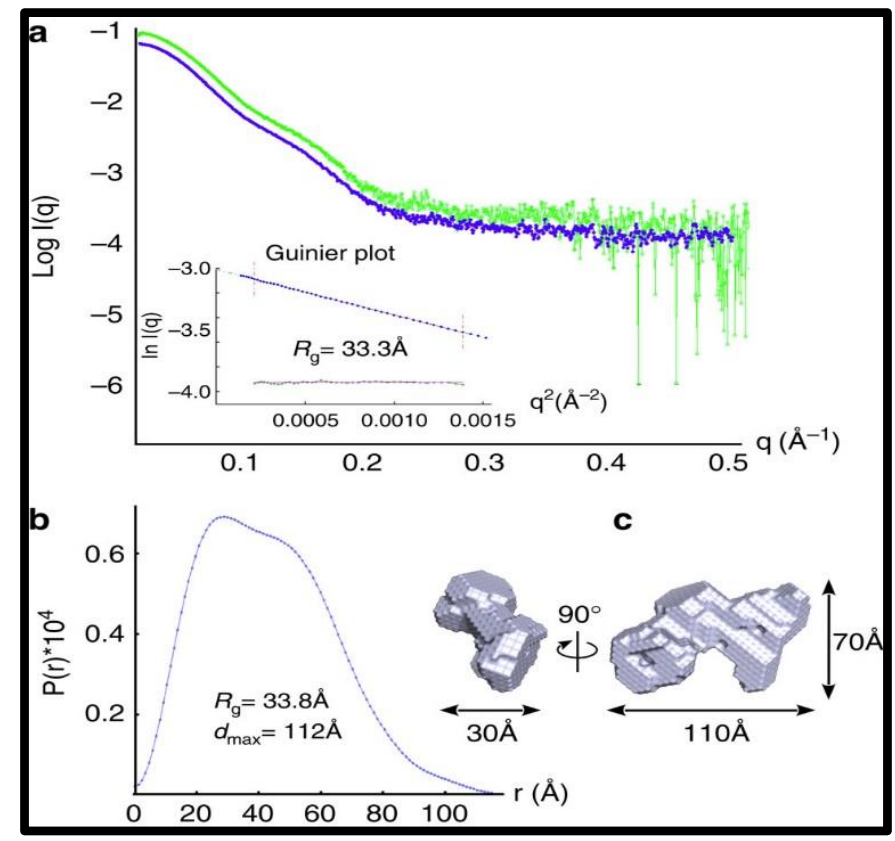

Figure 2.4: Determination of Radius of Gyration of polymer particles from SAXS

\section{data. $^{48}$}

The Radius of Gyration is directly related to the size and conformation that a polymer assumes when in a variety of states (i.e. solid, liquid), and the rapid determination of its value is part of what makes SAXS a very valuable technique. The derivation of the Radius of Gyration is summarized per Guinier's Equation:

$\ln [I(q)]=\ln \left(I_{0}\right)-\left[\left(q^{2} R_{g}^{2}\right) / 3\right]$

where $I(q)$ is the scattered intensity, $I_{0}$ is the incident X-Ray intensity, $q$ is the scattering vector, and $R_{g}$ is the Radius of Gyration in $\mathrm{nm}$. 
Prior to sending samples to Yale, the liquid crystalline nature was validated via observation of birefringence in polarized optical microscopy. Once all complexes were determined to be birefringent, small chunks of sample approximately $2 \mathrm{~mm}$ in diameter were carefully loaded with a plastic pipette tip into a glass SAXS capillary tube. Once the capillary tubes were loaded, they were carefully wrapped in cotton to protect the exterior surfaces from hitting against the plastic walls of a centrifuge tube in which the capillaries were placed. In general, the centrifuge tubes were spun at a rate of 3,000-4,000 revolutions per minute (rpm) in order to spread the sample uniformly across the interior of the capillary tube. Once it was decided that the samples had been spread uniformly along the interiors of the capillary tubes, the capillaries were sealed with glue, individually packaged, and sent out for SAXS analysis. 
3. Results and Discussion

\subsection{Dilute Solution Spectroscopy}

After centrifugation, observable phase separation occurred within the polymer and surfactant complexes such that solid polymer chunks were observed at the bottom of the vials. The supernatant, or liquid lying above the solid residue after centrifugation, was utilized for dilute solution spectroscopy studies. The viscosity and appearance of the complex hydrogel solution depended heavily upon the type of surfactant combined with P3KHT and the viscosity of the hydrogel increased in direct proportion to the amount of DHAB loaded into the system. Moreover, the appearance (namely the colors) of the solutions differed with respect to which surfactants were added. While a dilute solution of a C10 surfactant and P3KHT appeared orange, a dilute solution of a C14 surfactant and P3KHT was purple; such a trend reinforces that surfactants have a direct influence on the optical properties of the solution. As will be verified via UV/Visible and Fluorescence Spectroscopy, the electronic bandgap of the P3KHT/surfactant complex is dependent upon which type(s) of surfactant has been added. When surfactants with certain architectures, such as single tailed entities with long alkyl chains, are combined with P3KHT, red-shifted spectra with peak maxima greater than $600 \mathrm{~nm}$ are observed; these spectra suggest the presence of polymer chains which have transitioned into a rod-like morphology. Longer wavelengths of light (i.e. wavelengths of lower energy) are absorbed and fluoresced when the polymers assume rod-like conformations. In these conformations, the energy gap between the valence and conduction bands is reduced in 
comparison to systems of coiled morphology, since not as much photon energy is necessary to promote an electron to the conduction band.

A plethora of studies have been conducted to elucidate the effects of polymer conformation on the bandgap of a system. In one study, it was found that coplanarity of adjacent polymer chains works to minimize steric repulsions between the chains so that the effective $\pi$-conjugation length is maximized. ${ }^{49}$ Based on the data acquired from experimentation, it is desirable to learn more about the so called 'structure property' relationships between the physical arrangement of polymer chains and the system's electronic bandgap. The energy gap between the valence and conduction bands is most easily calculated from analyzing the optical absorption edge of the system, which is directly related to the energy difference between the ground and excited states as electromagnetic radiation is absorbed. ${ }^{50}$ Conventionally, the most widespread method of calculating bandgap from an optical absorption spectrum is to take the wavelength value at the onset of absorption, and then convert it to energy via Planck's equation (4) and equation 5:

$E(\mathrm{~J})=h c / \lambda(\mathrm{m})$

$E(\mathrm{eV})=(1239.84187 \mathrm{eV} \cdot \mathrm{nm}) /(\lambda(\mathrm{nm}))$

where $h$ is Planck's constant in $\mathrm{m}^{2} \cdot \mathrm{kg} / \mathrm{s}, c$ is the speed of light in $\mathrm{m} / \mathrm{s}$, and $\lambda$ is wavelength in $\mathrm{nm}$. The wavelength value at the onset of absorption can be found from the intersection of the extrapolations of the linear part of the spectrum. ${ }^{50}$ In Figure 3.1, the onset of absorbance is illustrated, wherein two tangential lines intersect at roughly $455 \mathrm{~nm}$; as such, this wavelength value is assigned to be the onset of absorbance. 


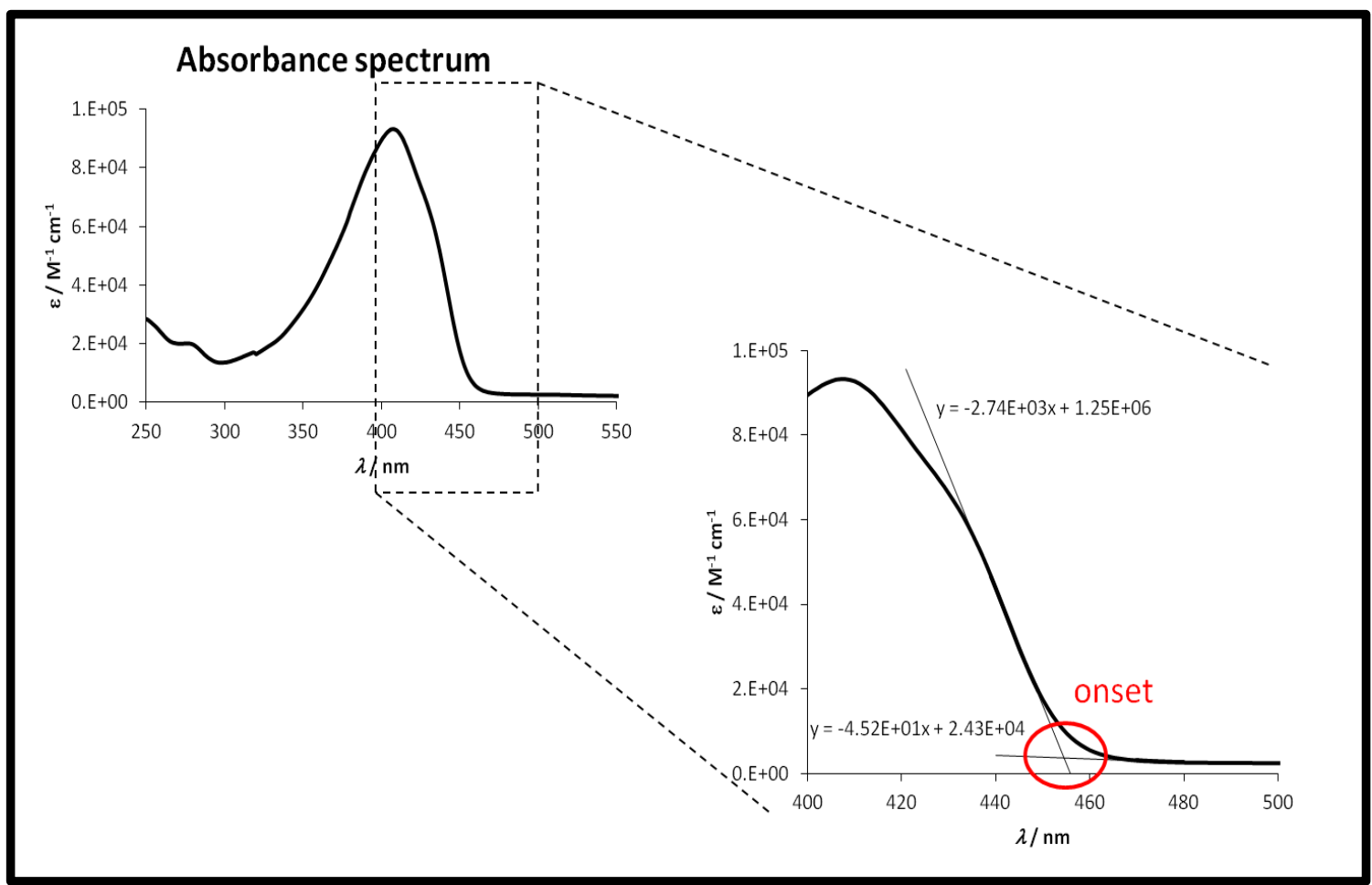

Figure 3.1: A conventional absorbance spectra. The wavelengths near roughly $455 \mathrm{~nm}$ represent the onset of absorption..$^{50}$

It is worthwhile mentioning that another simplistic method to determine bandgap is via cyclic voltammetry or differential pulse voltammetry. In cyclic voltammetry, a voltage is cycled from pre-defined minimum and maximum potentials, or vice versa. Oxidation (removal of electron from HOMO level) and reduction (addition of electron to LUMO level) are observed at two specific voltages, $V_{o x}$ and $V_{\text {red. }}$ The bandgap of the system can be found by computing the difference between the HOMO and LUMO levels, represented by $V_{o x}$ and $V_{r e d}$, and multiplying by the charge of an electron:

$E(\mathrm{eV})=e\left(V_{\text {red }}-V_{o x}\right)$

A blue shift in the UV/Vis spectra occurred whenever the amount of DHAB surfactant was increased with respect to CTAB or when the alkyl chain length of a single tailed 
surfactant was shortened. The UV/Vis spectra thus provide indirect evidence of the polymer chains adopting a more coiled morphology with a decreased effective conjugation length and an increased bandgap. We commence our investigation of surfactant architecture's influence on polymer chain conformation in dilute solution by analyzing the behavior of a dilute solution of P3KHT combined with $100 \%$ CTAB, as seen in Figure 3.2. The phenomenon of 'time dependent chromism,' or a change in the spectral and optical properties of the solution with respect to time, is seen to occur under certain conditions for this particular system.

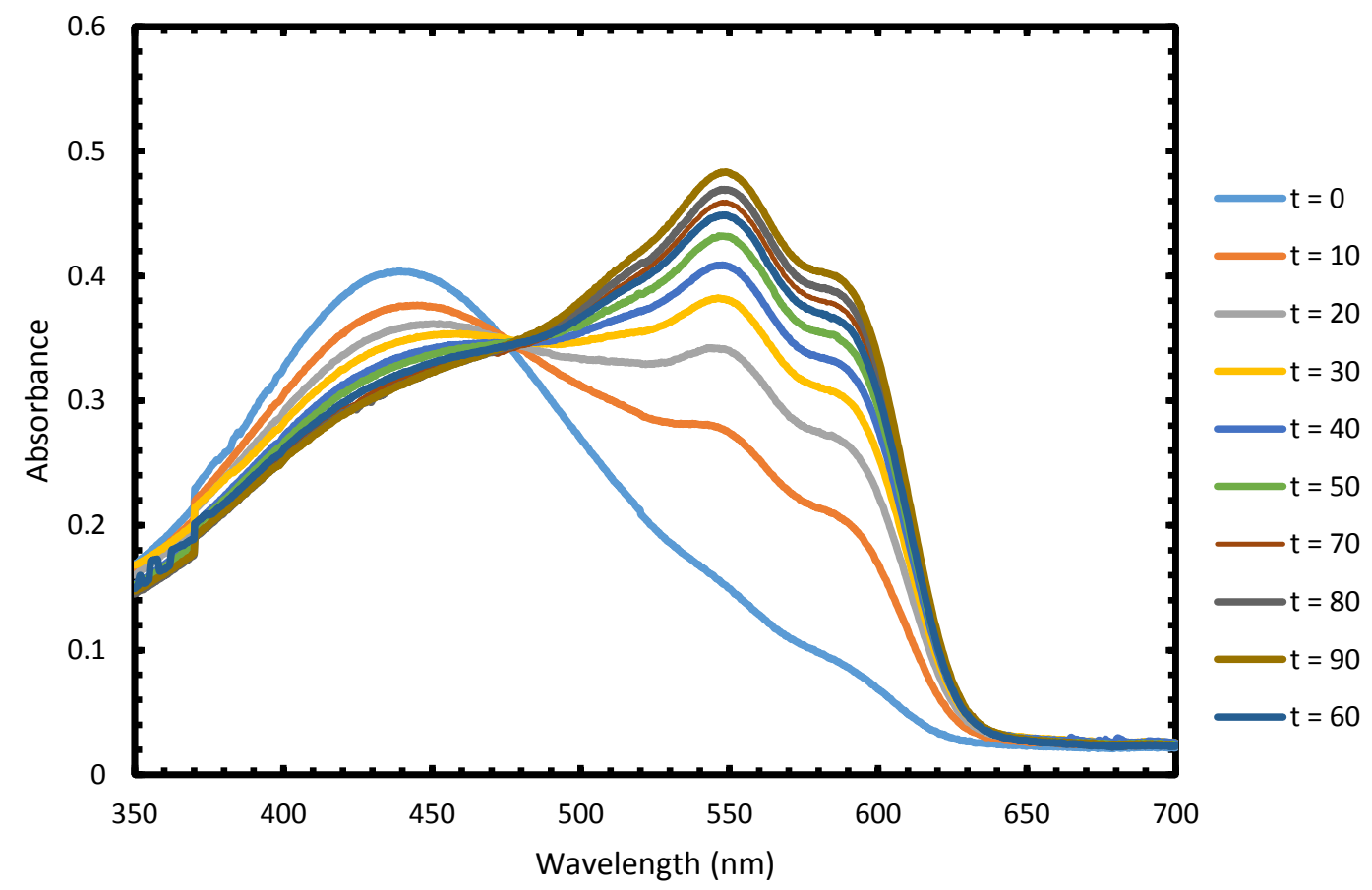

Figure 3.2: Absorbance vs. wavelength (nm) and time dependent chromism of a P3KHT/C16 (CTAB) complex. ' $t$ ' refers to time in minutes and the solution concentration is $0.133 \mathrm{mM}$. 
When CTAB (16 carbons in alkyl chain) is used as the surfactant for complexation, steric repulsions are more likely to occur between adjacent CTAB molecules as compared to surfactants of shorter alkyl chain lengths. CTAB chains occupy more unit volume than shorter surfactant chains, and thus mutual cationic charges repel one another more readily due to their increased steric crowding. Because it is not thermodynamically favorable to have molecules of the same charge in such close proximity, the CTAB chains tend to maximize their mutual separation to lower the system's free energy. The intercalation of the CTAB chains between the P3KHT chains, combined with increased separation between CTAB chains, contributes not to a compact coiled conformation of polymer/surfactant aggregates, but rather to an extended and ordered rod-like morphology. The thermodynamically based transition of the polymer chains from the coiled to the rod-like state occurs as a function of time, and it was found that the rate of this transition is inversely proportional to solution concentration. ${ }^{66}$ Based on the argument that the degree of steric repulsion increases as a function of surfactant alkyl chain length, it is easy to understand why the addition of CTAB induces a transition in which coiled chains assume rod-like structures. A compacted coil-like state simply cannot exist favorably with the higher levels of steric repulsion seen in a system with surfactants of long alkyl chain length, as increased steric repulsions between surfactants induce a greater degree of separation and long-range order between polymer chains.

In Figure 3.2, the initial absorption maximum $(t=0)$ occurs at approximately $440 \mathrm{~nm}$, but after roughly 30 minutes, the absorption maximum is seen to occur at $550 \mathrm{~nm}$. Such a spectral transition indicates that a system with a short conjugation length (i.e. of coiled morphology) is not sustainable for a long time due to the thermodynamically 
unfavorable interactions between CTAB chains in close proximity to one another. In order for the system to attain a lower free energy, the polymer chains adopt extended structures wherein the CTAB molecules become progressively more separated. The polymer chains become increasingly rod-like with longer effective conjugation lengths over the course of 90 minutes, as indicated by the successive increase in absorbance as a function of time in the red-shifted region of $550 \mathrm{~nm}$. If one were to illustrate the conformations which polymers assume over the course of time, the result would likely be of the following:

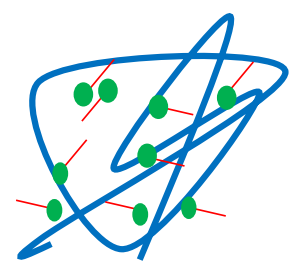

Morphology at $\mathrm{t}=0$

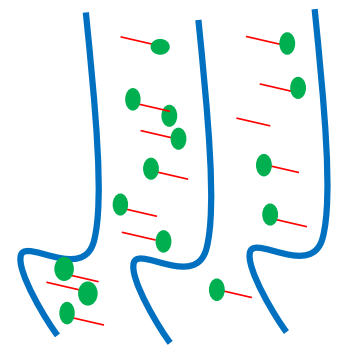

Morphology at $\mathrm{t}=30$

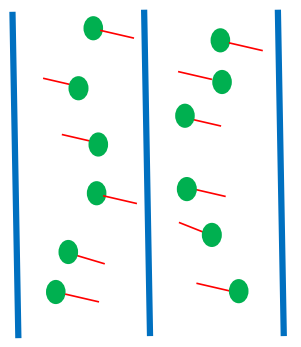

Morphology at $\mathrm{t}=90$

\section{Legend:}

$=$ surfactant

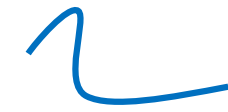

$=$ polymer chain .

\section{Figure 3.3: Hypothetical polymer/surfactant conformations as a function of time.}

Although more advanced characterization techniques such as SAXS should be performed to best elucidate polymer chain conformation upon surfactant complexation, the spectral changes seen in the UV/Vis spectrum as a function of time are very meaningful in that the system's bandgap progressively decreases with time per Figure 3.2. As was previously discussed, a reduction in energy bandgap is directly proportional to an increased effective conjugation length in the polymer (i.e. polymers that are more 
rigid in character have smaller bandgaps). The approximate values for polymer bandgap as a function of time are displayed in Table 3; all bandgap values are calculated from Planck's Equation. The wavelengths substituted into the equation stem from the values at which the onset of absorption was deemed to occur per Figure 3.1.

Table 3: Approximate Bandgaps in eV of a P3KHT/CTAB Complex at Various Times (Minutes) from Figure 3.2.

\begin{tabular}{|c|c|c|}
\hline Time (min) & Approximate $\lambda_{\text {onset }}(\mathrm{nm})$ & Bandgap $E_{g}(\mathrm{eV})$ \\
\hline 0 & 510 & 2.431 \\
\hline 10 & 540 & 2.296 \\
\hline 20 & 560 & 2.214 \\
\hline 30 & 570 & 2.175 \\
\hline 40 & 600 & 2.066 \\
\hline 60 & 610 & 2.033 \\
\hline 90 & 625 & 1.984 \\
\hline
\end{tabular}

It should be noted that the wavelengths representative of an absorption onset are approximate and are therefore subject to a certain degree of uncertainty. Nevertheless, efforts were made to optimize the accuracy of the onset wavelengths by utilizing tangent approximations in Excel software. Based on Table 2, there is a progressive decrease in approximate bandgap for the P3KHT/CTAB complex as a function of time. Levels of uncertainty in bandgap values are the same for all reported times, and thus the ratio of 
one bandgap with respect to the other will remain unchanged regardless of the level of uncertainty. As can be inferred from Table 2, there is an $18.5 \%$ reduction in bandgap for the P3KHT/CTAB system after 90 minutes at $25^{\circ} \mathrm{C}$ and $0.133 \mathrm{mM}$ concentration. Within the first 30 minutes after dilution, the bandgap has decreased by roughly $0.26 \mathrm{eV}$ and the maximum absorbance is within the red-shifted region of $550 \mathrm{~nm}$. For a solution of this particular concentration, 30 minutes is the estimated time period necessary for the polymer chain conformations to be predominately of the rod form.

Our attention will now be focused towards assessing the effects of increased DHAB loading on the 'coil to rod' transition. In Figure 3.4, it can be seen that a complete conformational change from coil to rod does occur, despite the fact that $5 \%$ of the surfactant (by mols) is comprised of DHAB. The transition from coil to rod (i.e. from the low to high wavelength regions) actually occurs faster than was seen in Figure 3.2 for the pure CTAB system; one can justify this anomaly based on the fact that the solution concentration of $0.133 \mathrm{mM}$ might have been inaccurate. For instance, the concentration might have been as low as $0.07 \mathrm{mM}$ in actuality, thereby allowing for a much faster coil to rod transition to have occurred based on the principle of an inverse first order relationship between concentration and transition rate. ${ }^{51}$ The postulated value of $0.07 \mathrm{mM}$ is based on the belief that in some instances, half of the pipette's contents might have remained aggregated to its walls following an ejection. Inaccuracies in solution concentration may be attributed to incomplete ejections of the hydrogel from the pipette into the water solution (i.e. if injecting $50 \mu \mathrm{L}$ of the hydrogel into the water was desired, perhaps only $40-45 \mu \mathrm{L}$ was actually delivered). Attempts were made to mitigate incomplete ejections by lowering the viscosity of the hydrogels through serial dilutions, since higher viscosity 
gels easily aggregated to the walls of pipette tips. A complete transition to a rod-like state does occur for a $5 \%$ DHAB system, as the wavelength at maximum absorbance $(550 \mathrm{~nm})$ is the same as the wavelength of maximum absorbance for the CTAB system after approximately 30 minutes.

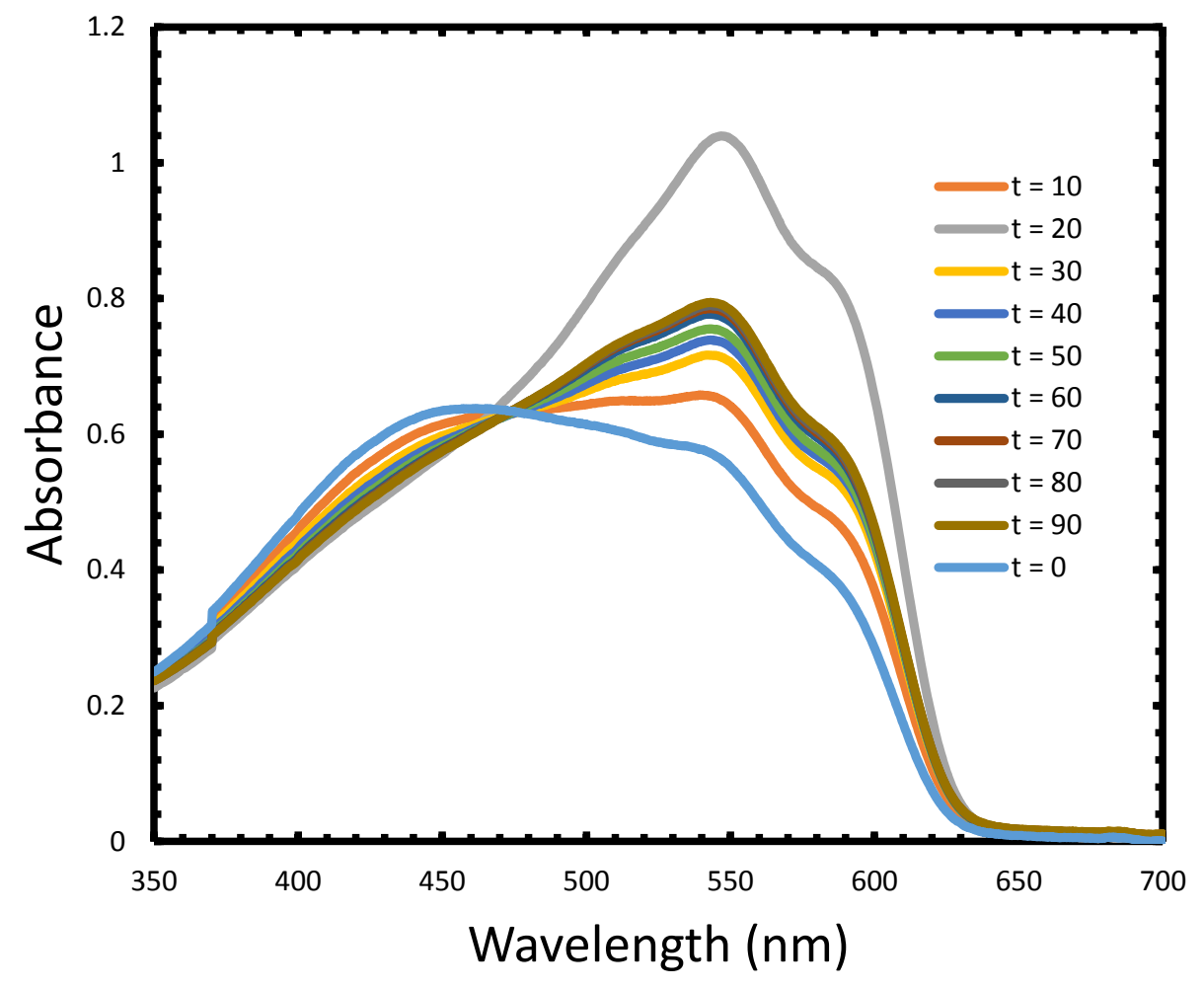

Figure 3.4: Time dependent chromism of a P3KHT/5\% DHAB Complex. ' $t$ ' is time in minutes.

As the amount of DHAB is increased beyond 5\%, a stark difference is seen in the UV/Vis spectra. Time dependent chromism does not occur, since the wavelength of maximum absorbance remains at or below 510nm even after several days. The lack of 
time dependent chromism indicates that increasing the abundance of $\mathrm{DHAB}$, a doubletailed surfactant, impedes the formation of polymer rods. Double tailed surfactants have been utilized for aggregate formation of nanorods, nanoparticles, and other systems at the nanoscale. Different aggregate structures with an assortment of packing geometries may result when double-tail surfactants are employed. ${ }^{52}$ Cylindrical, disk, spherical, or vesicular polymer structures are only a few of many various polymer aggregate architectures that can result from the addition of a double-tailed surfactant. ${ }^{52}$ In another study, the addition of DHAB to a polymer solution of poly(ethylenimine) led to an exceptionally compacted polymer conformation wherein flexibility of the polymer backbone was substantially increased. Aggregated hydrophobic moieties with small radii of gyration were formed and the hydrocarbon to water interfacial area was minimized upon DHAB addition. ${ }^{53}$ The UV/Vis spectra acquired for increased DHAB loadings, presented in the Figures to follow, is suggestive of the theory that compact and aggregated moieties form, since the spectra are significantly blue-shifted. If one were to perform SAXS on the DHAB containing complexes, data representative of compacted and coiled polymer architectures with a small radius of gyration $\left(R_{g}\right)$ should arise. 


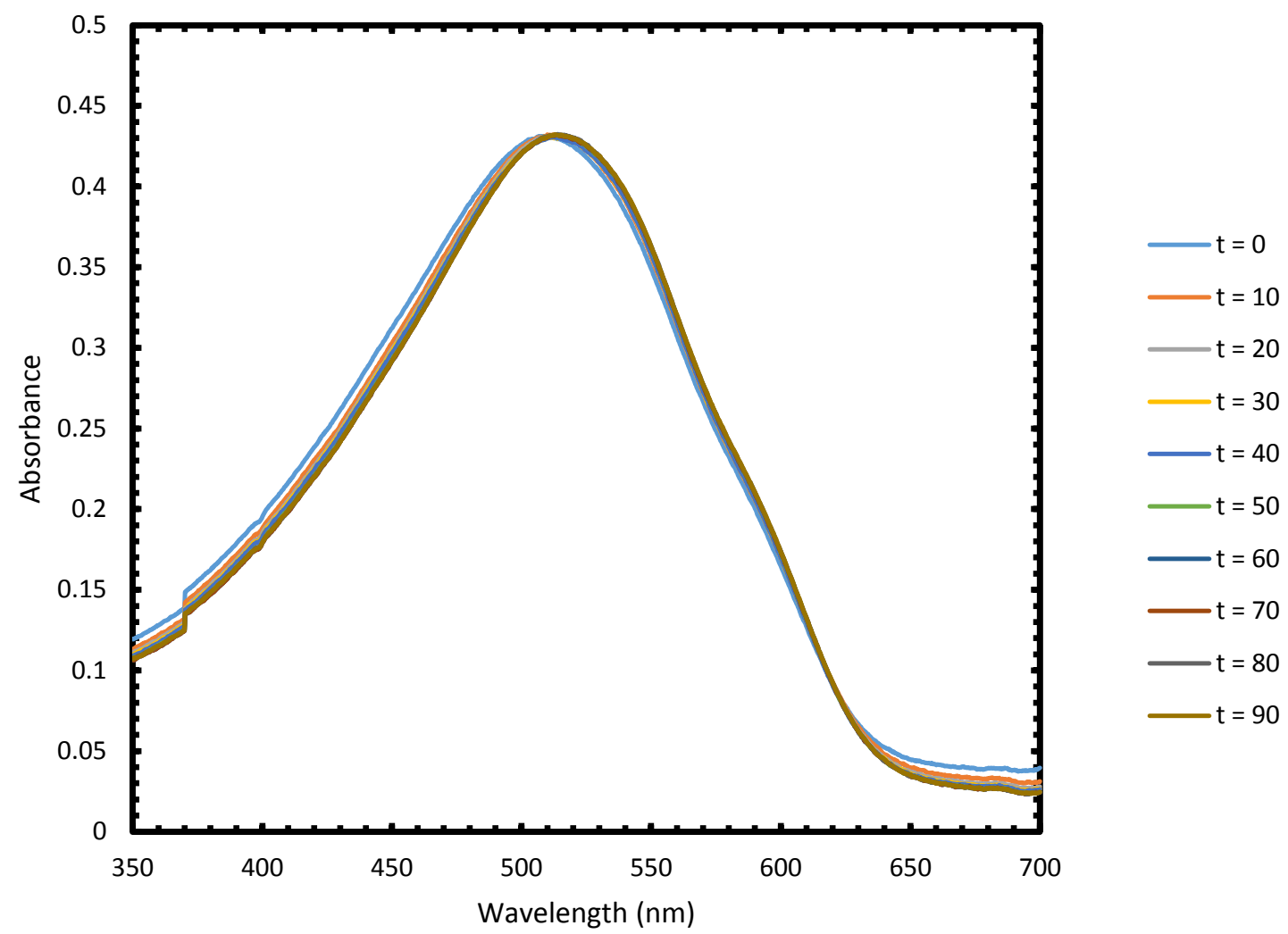

Figure 3.5: UV/Vis spectra of a P3KHT/10\% DHAB solution. There is no change in chromic properties with respect to time.

In Figure 3.5, it is apparent that only an infinitesimal amount of red-shifting occurs after 90 minutes; moreover, this solution exhibited the same UV/Vis profile after several days. In contrast with the time dependent chromism seen for the P3KHT/5\% DHAB solution, there must exist a critical concentration of DHAB at which a coiled polymer morphology becomes more favorable thermodynamically. Greater amounts of DHAB are likely to cause drastic increases in the hydrophobicity of the system such that nanodomains of compacted polymer aggregates form. Since the P3KHT chains are bound to the DHAB via ionic interactions, any changes in conformational structure of DHAB aggregates are imparted to the polymer chains. Thus, because adding more DHAB 
increases hydrophobicity and hence compactness, the P3KHT chains invariably adopt disordered and condensed 'coiled' orientations. In Figure 3.6, the UV/Vis profiles of P3KHT/20\% DHAB and P3KHT/30\% DHAB solutions are represented. Only one spectrum was obtained for each complex because it was determined that absolutely no degree of time dependent chromism occurred (i.e. the wavelength of maximum absorbance will be in the same location even after several days).

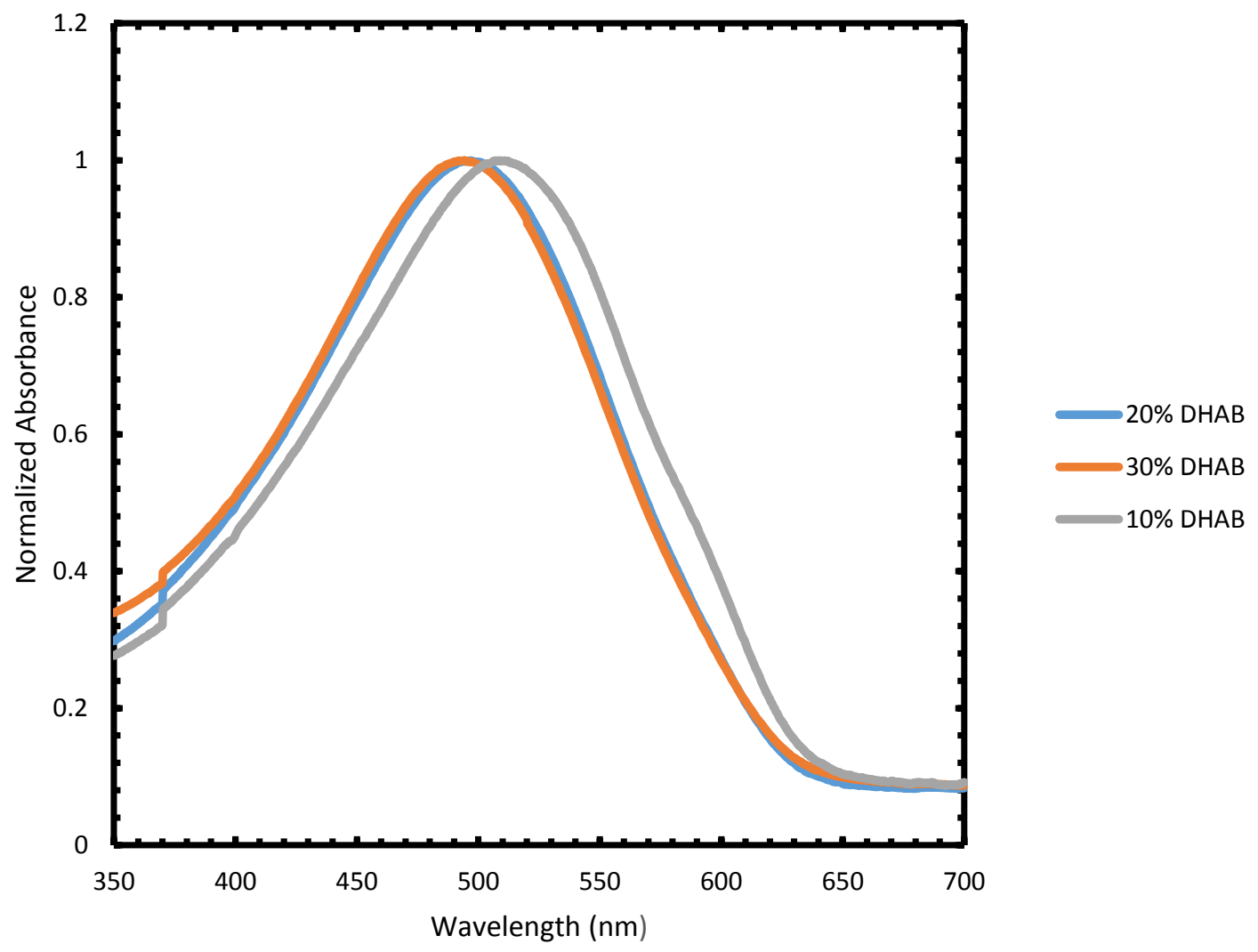

Figure 3.6: UV/Vis spectra of 10, 20, and 30\% DHAB complexes. No time dependent chromism occurs for this level of DHAB loading.

The trends in the UV/Vis spectra for complexes of varying DHAB loading were supported by emission spectra. In the figures to follow, a time dependent red-shifting in 
the emission wavelengths is seen for all complexes which displayed time dependent chromism. Moreover, in the non-normalized emission plots, a progressive quenching of emission intensity is seen, which can be attributed to an aggregation of the polymer/surfactant network. The lower photoluminescent intensity might also be attributed to the fact that that excitation wavelength was not adjusted for complexes that had more blue-shifted spectra. As such, the lower molar absorptivities recorded for these complexes might well be attributed to the lower observed emission intensities. Although quenching is seen with respect to time for polymer/surfactant complexes, complete quenching is only observed for pure P3KHT solutions - it is believed that the strong $\pi-\pi$ interactions between adjacent polymer backbones negate all fluorescence, as intermolecular charge transfer due to aggregation causes a non-radiative response to excitation. ${ }^{24,25}$ In Figure 3.7, the emission intensity for a P3KHT/5\% DHAB solution is red-shifted to $620 \mathrm{~nm}$, indirectly supporting the datum that this complex has a smaller energy gap between the valence and conduction bands and that the polymer chains selfassemble into rod-like conformations. The emission of longer (i.e. lower energy wavelengths) directly translates into a smaller bandgap via equation 4 . 


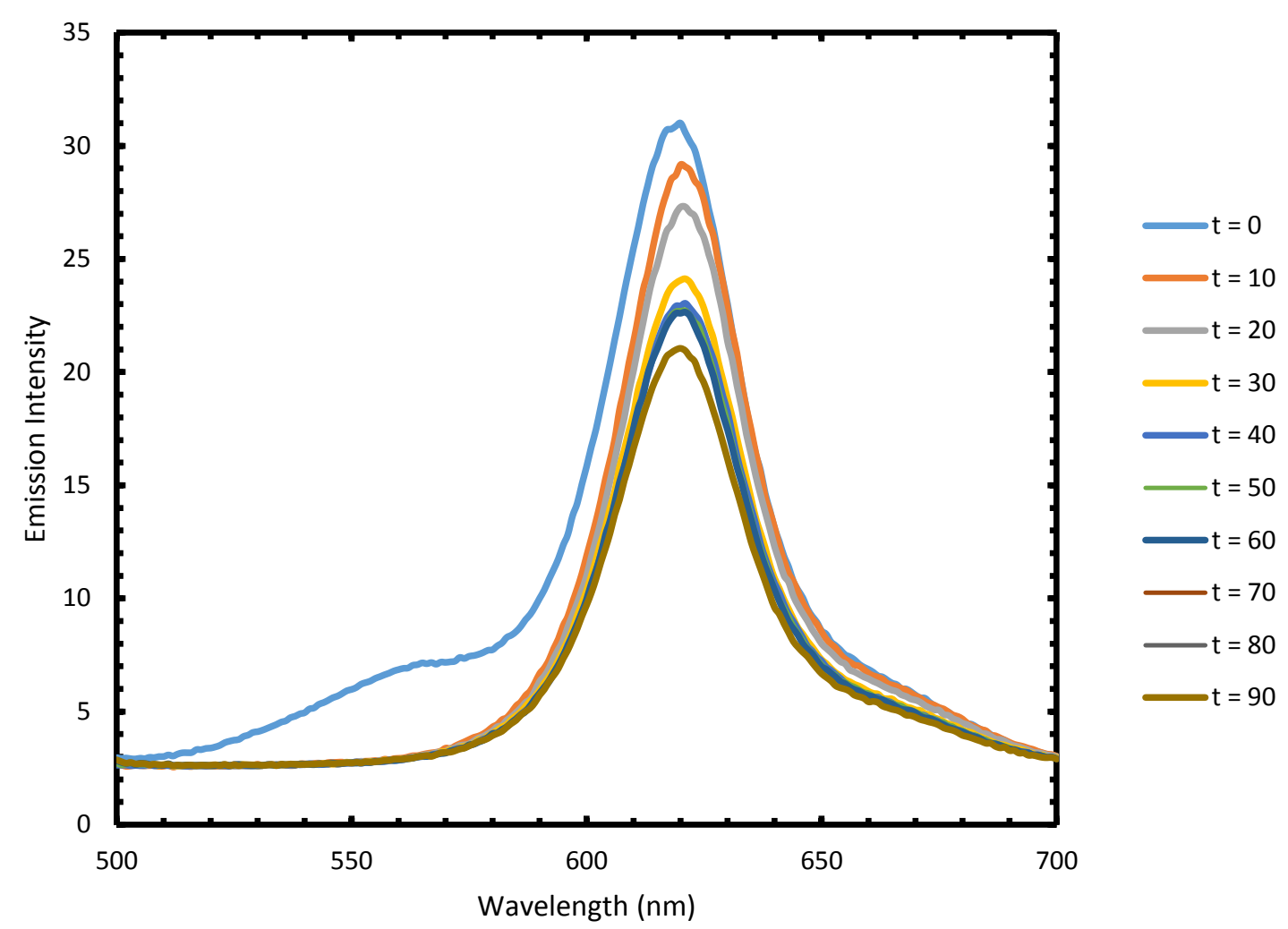

Figure 3.7: Emission spectra for a P3KHT/5\% DHAB solution. All emission wavelengths are above $600 \mathrm{~nm}$ and quenching increases with respect to time.

The wavelengths of maximum absorbance seen in Figure 3.7 should be compared to those displayed in Figure 3.8, which represent the normalized emission profiles for 10, 20, and 30\% DHAB/P3KHT solutions, respectively. In Figure 3.8, the greatest wavelength in which emission was seen to occur was at $590 \mathrm{~nm}$. One should note that the emission wavelength for a P3KHT/10\% DHAB solution occurred at 570nm, which is a shorter emission wavelength than for 20 and 30\% DHAB solutions. This result is counterintuitive, as lower amounts of DHAB should contribute to a smaller bandgap and longer emission wavelengths in theory. However, one must realize that there is an inherent uncertainty with regard to each solution's concentration and molar ratio of 
DHAB: CTAB, along with measurement uncertainties stemming from the fluorimeter used to obtain the spectra. The difference in emission wavelengths (590nm vs. $570 \mathrm{~nm})$ between the 20 and 30\% DHAB solutions and the 10\% DHAB solution thus might not be due to conclusive differences in chain conformation, but to inherent uncertainties of the instrument or of the solution concentrations.

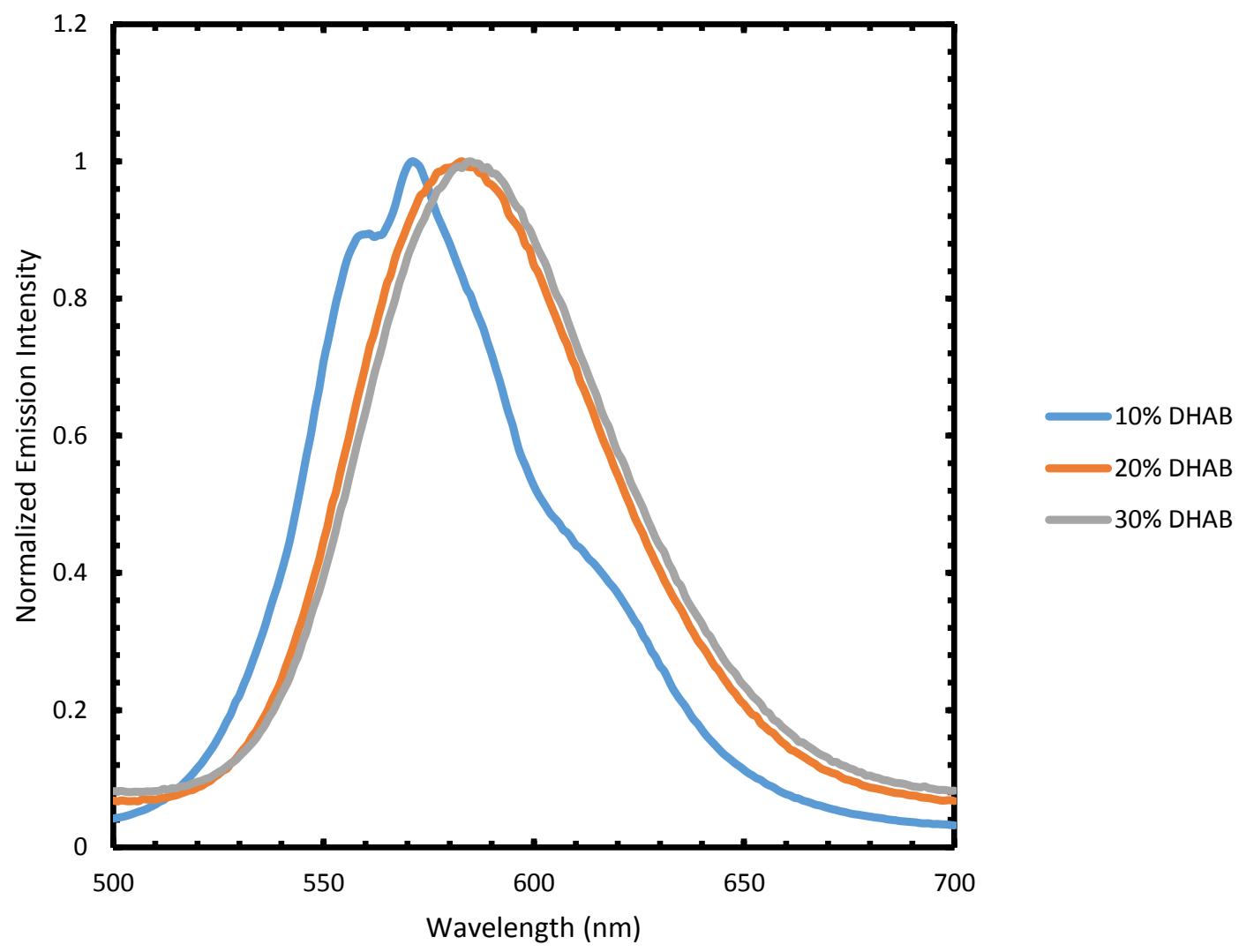

Figure 3.8: Normalized Emission spectra for 10, 20, and 30\% DHAB/P3KHT solutions.

As the optical and emissive properties of the P3KHT/surfactant complexes have been discussed when the surfactant is either comprised of pure CTAB or of a combination of $\mathrm{CTAB}$ and $\mathrm{DHAB}$, it is now necessary to evaluate and discuss the 
UV/Visible and Emissive data obtained from complexes with a single tailed surfactant of varying alkyl chain length. When a surfactant is complexed with polymer, a variation of the surfactant alkyl chain length can lead to differences in the dispersive, thermal, and dynamic mechanical properties of the network, in addition to other changes. In a study by Musking et. al., increasing the alkyl chain length of a sodium dodecyl sulfate surfactant in combination with an LDPE/LDH polymer composite led to a successive increase in the $d$-spacing between the $\langle 003\rangle,\langle 006\rangle$, and $\langle 009\rangle$ families of crystallographic planes. ${ }^{54}$ Moreover, the study by Musking et. al. also found that intercalated structures formed between the polymer and surfactant whenever the surfactant alkyl chain length was equal to or greater than 12 carbons. Conversely, shorter surfactant alkyl chains contributed to larger aggregate structures with enhanced mobility between polymer chains- - this trend is consistent with the theory that shorter alkyl chains present less steric hindrance and electrostatic repulsion, allowing for the polymer/surfactant network to have less rigidity as a whole and to thus form a coiled morphology. Crystallite size, decomposition temperature, and crystallization temperature were all observed to increase as a function of surfactant alkyl chain length. Moreover, SEM micrographs depict more strongly connected fibrillary networks for systems of longer alkyl chain length. ${ }^{54}$ As will be discussed shortly, increasing the alkyl chain length of a surfactant combined with P3KHT will have a similar effect to that observed in the studies of Musking, et. al., as more rigid and fibrillary morphologies are attained.

The results of dilute solution spectroscopy for C10, C12, C14, and C16 (CTAB) combined with P3KHT suggest that longer alkyl chains contribute towards greater redshifting and hence smaller electronic bandgaps. An interesting phenomenon occurred in 
the experiment in that the $\mathrm{C} 14$ complex exhibited a red-shifted absorption peak at approximately 540nm at $\mathrm{t}=0$. This result suggests that there was no time dependent chromism for the $\mathrm{C} 14$ complex, as the chromic transition was instantaneous. There may be considerable differences in the aggregation and de-aggregation behaviors between the polymer chains and surfactant moieties as a function of surfactant alkyl chain length-the data for the C14 complex suggests that little to no deaggregation of the P3KHT chains occur upon $\mathrm{C} 14$ addition, while significant deaggregation (blue-shifting) occurs upon the addition of $\mathrm{C} 16$ (CTAB). A discussion of the factors relating surfactant architecture to polymer aggregation mechanisms is beyond the scope of this report, as the causes for the differing behaviors have only been postulated. In Figure 3.9, it is intuitive that the maximum absorbance wavelengths become increasingly red-shifted as surfactant alkyl chain length increases. One should also notice that the curve representative of the C16 complex was obtained after 90 minutes; the $\mathrm{C} 16$ curve at $\mathrm{t}=0$ exhibited an absorbance maximum at roughly $480 \mathrm{~nm}$. Thus, CTAB was the only surfactant seen to induce time dependent chromism. The long length of the CTAB alkyl chain (16 carbons) likely induces a significant degree of steric and electrostatic repulsions, thereby prohibiting the formation of compacted and or coiled domains; as such, the polymer chains transition from a coiled to rod state as a function of time. This transition is thermodynamically driven, as the exothermic reduction in enthalpy outweighs the decrease in entropy so as to minimize free energy. 


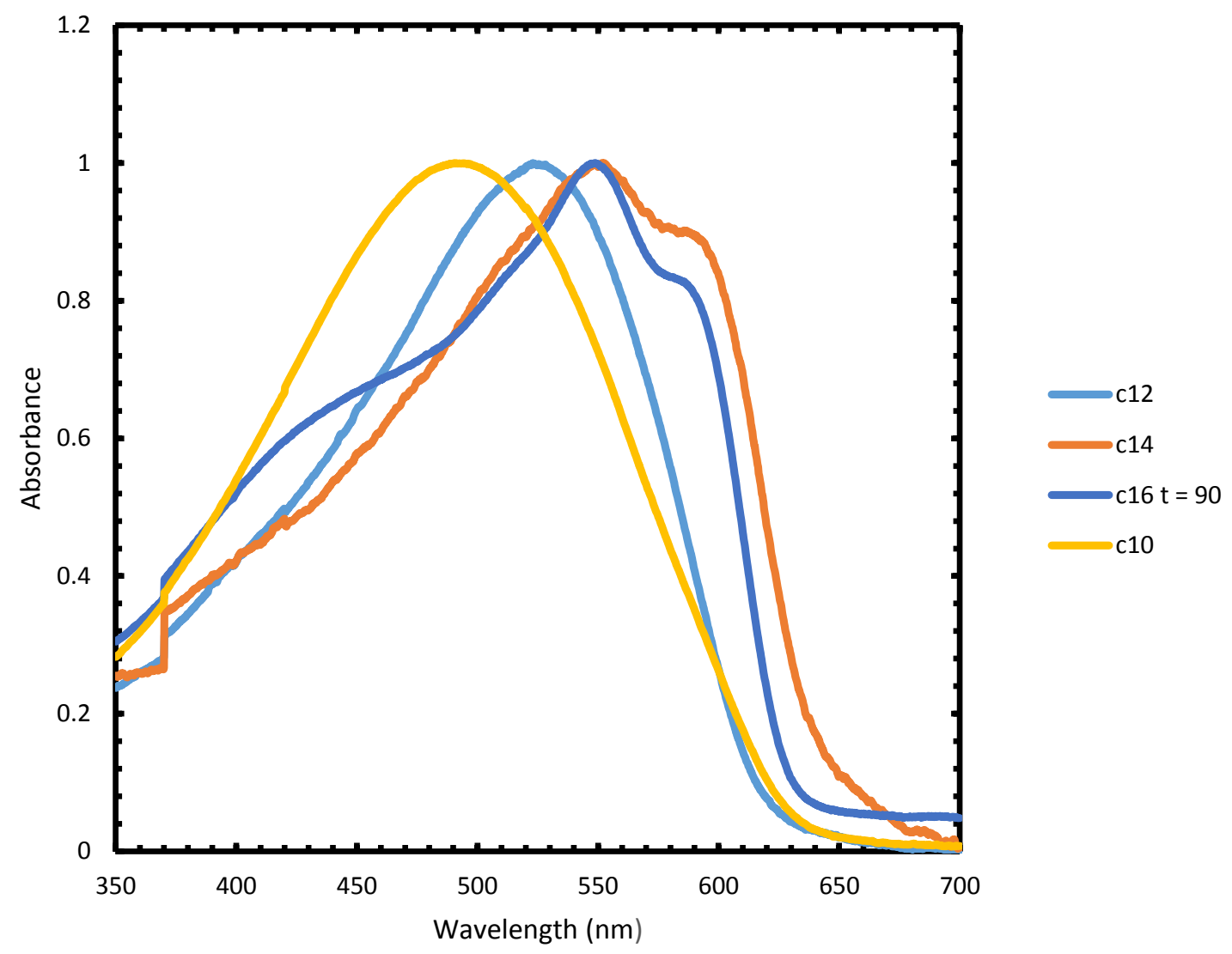

Figure 3.9: Normalized Absorbance spectra for P3KHT/C10, C12, C14, and C16 solutions.

The data in Figure 3.9 provide indirect evidence for increased effective conjugation lengths (ECLs) for systems of surfactants with longer alkyl chains. As the alkyl chain of a cationic surfactant increases, the morphology of the resulting complex is greatly affected-polymer pillars or honeycombs have been observed for systems where the surfactant length is greater than or equal to 12 carbons and the adsorption efficiency of these systems is significantly greater due to greater separation between polymer chains and hence larger pore volumes. ${ }^{55}$ Polymer pillars refer to uniformly aligned polymer 
chains that are orthogonal to the substrate onto which they are grafted, and honeycomb structures result when the polymers align into hexagonal packing geometries.

The variation of surfactant alkyl chain length directly influences the P3KHT/surfactant emissive properties. As seen in Figure 3.10, the emission profile is consistent with the absorbance profile in Figure 3.9 in that there is a progressive red-shift in the spectra with respect to surfactant alkyl chain length. The differences between wavelengths of maximum emission intensity are more subtle in Figure 3.10 as compared to the differences between wavelengths of maximum absorption intensity in Figure 3.9; this trend is most likely due to the fact that each complex has a unique Stokes' shift. Despite each complex having dissimilar Stokes' shifts, it is evident in Figure 3.10 that as the alkyl chain length of a single chain surfactant is increased, the emission wavelength progressively increases, indicating the presence of both lower energy photons and a smaller energy gap between the valence and conduction bands. 


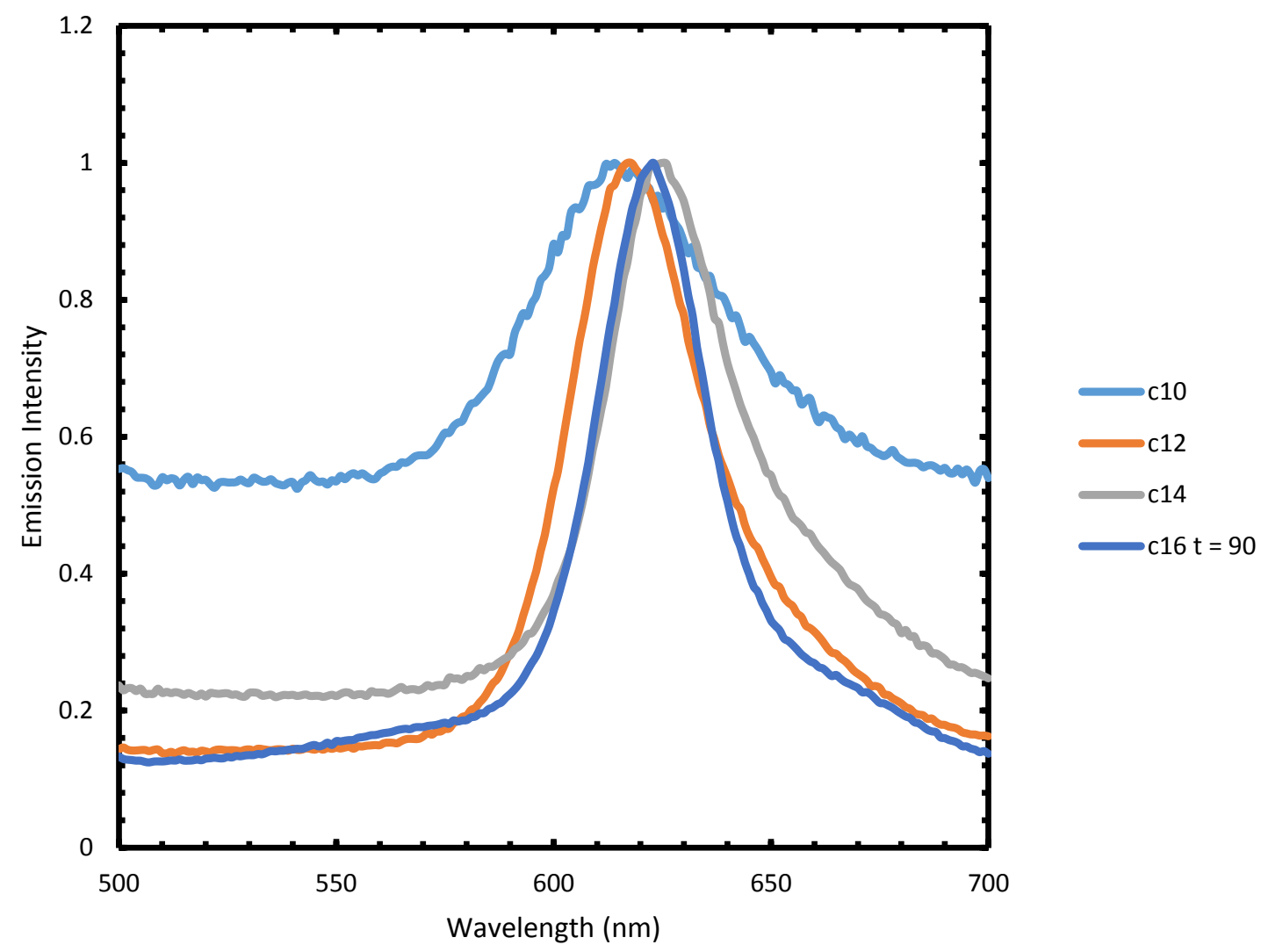

Figure 3.10: Normalized Emission Intensity for P3KHT/C10, C12, C14, and C16 solutions.

\subsection{Dynamic Light Scattering (DLS) Studies}

Attempts were made to deduce mean particle or aggregate sizes for various polymer/surfactant complexes via performing Dynamic Light Scattering (DLS). While some notable trends exist in the data (i.e. particle size increasing as a function of DHAB loading until 20\% DHAB), anomalies exist which do not make theoretical sense. For instance, the mean particle size decreases as the alkyl chain length of surfactant increases from 14 to 16 carbons, or as the percent DHAB loading increases from $20 \%$ to $30 \%$. Because the laser wavelength for the DLS instrument is fixed at $633 \mathrm{~nm}$ (i.e. within the 
absorbance range of a conductive polymer), not all light incident upon the sample is scattered and a certain percentage is absorbed. The fact that the conductive polymer samples absorb a portion of the light from the lasing source means that the validity of the DLS data is easily compromised, since a reduction in the sample's light scattering cannot be solely attributed to particle size effects. Despite these difficulties encountered in DLS, the acquired data is displayed in Table 4.

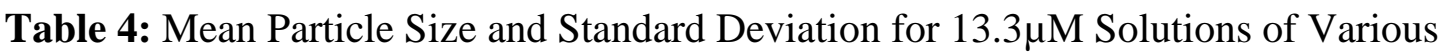
Complexes as Determined via Dynamic Light Scattering (DLS).

\begin{tabular}{|c|c|c|}
\hline $\begin{array}{c}\text { Identity of } \\
\text { Polymer/Surfactant } \\
\text { Complex }\end{array}$ & Mean Particle Size (nm) & Standard Deviation (nm) \\
\hline P3KHT/C10 & & \\
\hline P3KHT/C12 & 55.08 & 7.763 \\
\hline P3KHT/C14 & 245.514 & 60.834 \\
\hline P3KHT/C16 (CTAB) & 631.15 & 519.959 \\
\hline Pure P3KHT & 87.107 & 18.377 \\
\hline No Surfactant) & 105.226 & \\
\hline P3KHT/5\% DHAB & & 10.992 \\
\hline P3KHT/10\% DHAB & 105.857 & 11.056 \\
\hline P3KHT/20\% DHAB & 161.105 & \\
\hline P3KHT/30\% DHAB & & \\
\hline
\end{tabular}


The standard deviation observed for the $\mathrm{C} 14$ complex is much larger than the deviation seen for the other complexes; although the measurements were repeated for C14 multiple times and different concentrations were also tested, a very large standard deviation in the mean particle size still resulted. A possible explanation for the large deviation might be due to the colloidal nature of the $\mathrm{C} 14$ solution - in many cases, complete solubility of the complex did not occur. When there are concentrated regions of large macromolecules (i.e. colloidal assemblies) interspersed throughout a solution that is otherwise free of large molecules, there is a high probability that the average light scattering intensity across the entire solution will vary significantly.

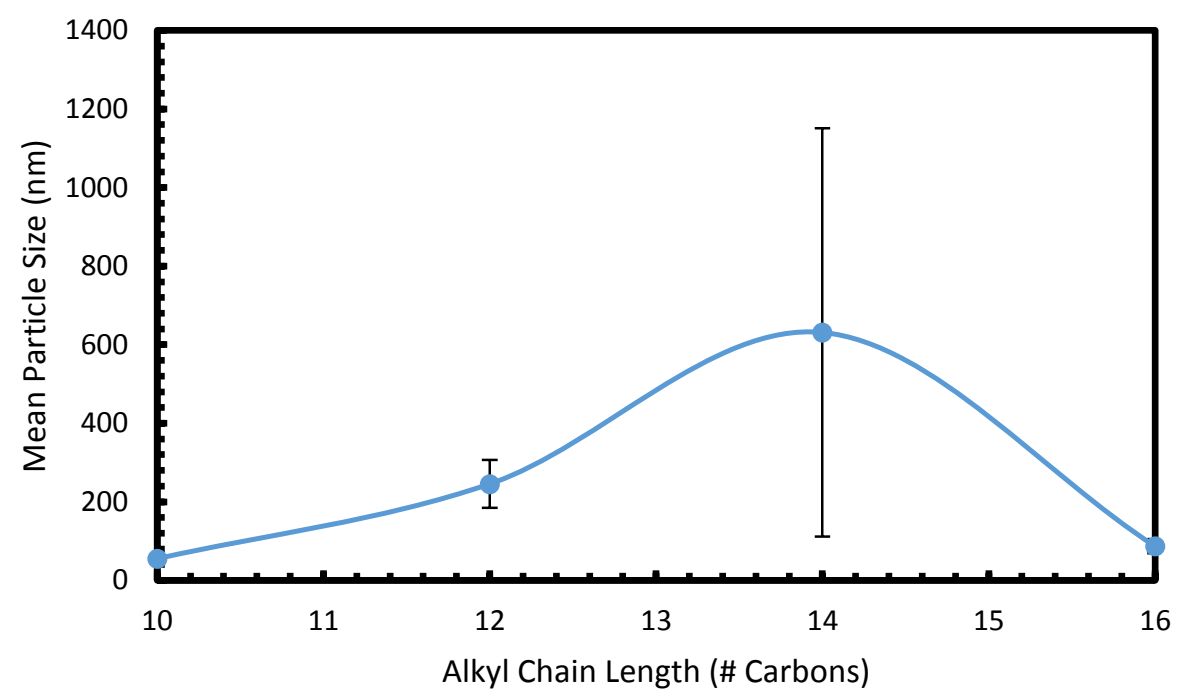

Figure 3.11: Mean particle size vs. alkyl chain length. There is a reduction in particle size for chains greater than 14 carbons.

In Figure 3.11, one can notice the abnormally large standard deviation in mean particle size for the $\mathrm{C} 14$ complex, as well as the reduction in particle size for alkyl chains longer than 14 carbons. The progressive increase in particle size with respect to alkyl 
chains 10 to 14 carbons long agrees well with theory because increased steric and electrostatic repulsions occur as the alkyl chain is increased, thus favoring a greater separation between chains. This principle contributes to an increase in mean particle size, although the particle size shrinks for alkyl chains longer than 14 carbons - the source of this effect is beyond the scope of this discussion and it is merely mentioned as an unexpected trend. In Figure 3.12, the mean particle size increases drastically from 5 to 10\% DHAB content, levels off between 10 and 20\% DHAB, and decreases gradually from 20 to 30\% DHAB. The standard deviations in particle size are very similar for all DHAB loading ratios and the absolute difference in particle size (maximum observed minimum observed) is a mere $85 \mathrm{~nm}$, as compared to an absolute difference of nearly 550nm in Figure 3.11. If this data could be confirmed through reproduction, it is evident that changing the surfactant alkyl chain length has a much more profound effect on altering the mean particle size as compared to changing the DHAB loading ratio.

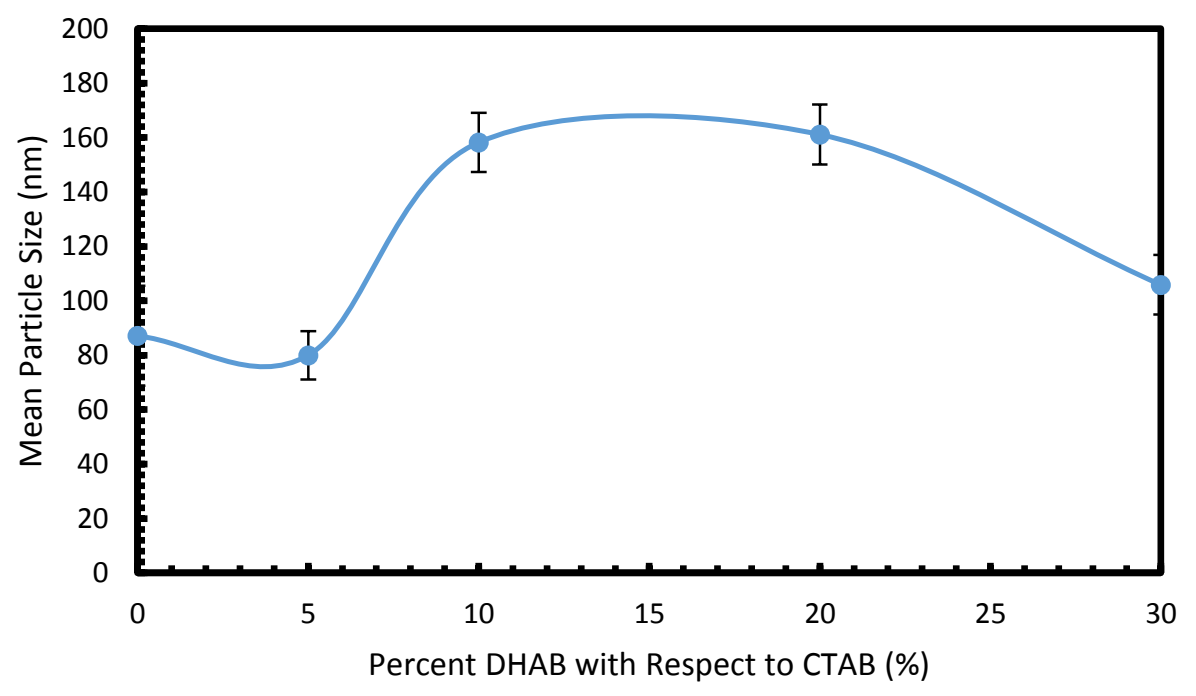

Figure 3.12: Mean particle size vs. percent DHAB surfactant in system. 


\subsection{Properties of Concentrated Solutions}

In addition to tailoring the morphology of conjugated polyelectrolytes in the dilute state, significant efforts have been made to control morphology and the degree of crystallinity in more concentrated solutions. The so called 'lyotropic ordering' of polymers can occur based on the formation of micelles of surfactant in solution. Since the conjugated polyelectrolytes are ionically bound to the surfactants, any changes in surfactant structure or ordering will be imparted to the CPEs. Above a specific concentration termed the critical micelle concentration, or $\mathrm{CMC}$, micelle formation becomes thermodynamically favorable in that the entropic penalty of assembling the surfactant molecules is less than the entropic penalty of exposing the hydrophobic portions of surfactant monomers with water. Micelles may assume different architectures depending on the surfactant packing parameter, which is directly influenced by the concentration of surfactant. As the cross sectional area of the hydrophilic core of the surfactant aggregate decreases with added surfactant, the packing parameter increases, and as a result, bilayers of polymer/surfactant complexes with lamellar morphology may form at sufficiently high solution concentrations. Although a lyotropic liquid crystalline polymer phase may form as a result of many different variables at sufficiently high concentrations, a very important variable is surfactant architecture. In Figure 3.13, the phase behavior is depicted as a function of both concentration and DHAB loading ratio. For instance, ' $90 \%$ CTAB' refers to a system with a 9:1 molar ratio of CTAB to DHAB, while '70\% CTAB' refers to a system with a 7:3 molar ratio of CTAB to DHAB. For any given $\mathrm{DHAB}$ loading ratio, it is intuitive that higher concentrations favor the formation of the nematic lyotropic liquid crystalline phase - this trend is in direct agreement with 
Onsager's theory for rigid rod formation. In Onsager's theory, a decrease in excluded volume between chains leads to an increase in packing entropy; this increased packing entropy outweighs the decrease in orientational entropy. ${ }^{56}$

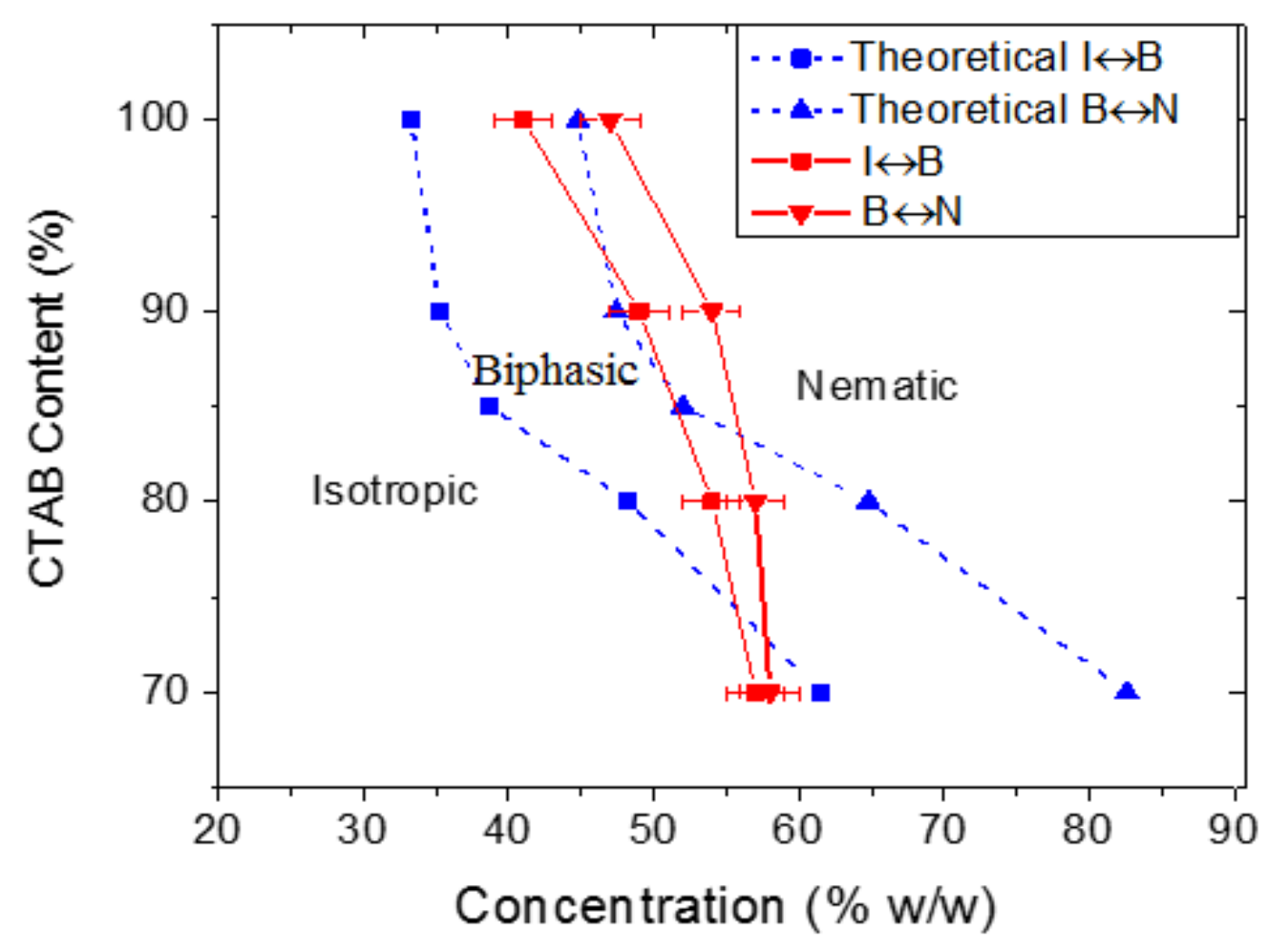

Figure 3.13: Phase behavior of P3KHT/Surfactant solutions as a function of concentration. ${ }^{66}$

It should be noted that the morphology of the complex does not instantaneously change from a disordered isotropic state to a highly ordered and nematic liquid crystalline phase, for there exists a small 'biphasic' regime. The biphasic region is simply a blend of the isotropic and nematic liquid crystalline phases; birefringence occurs, but only within small nanodomains interspersed throughout the area being analyzed. In the nematic 
phase, complete birefringence occurs. In the schemes and figures to follow, illustrations are presented of the isotropic, biphasic, and nematic liquid crystalline phases. It can be readily observed that birefringence does not occur upon rotation of the polarized light for the isotropic phase, and that birefringence only partially occurs for the biphasic phase. A very strong birefringence occurs for a sample in the nematic liquid crystal phase upon rotation of cross polarized light. In Figure 3.17, $c^{*}$ can be substituted for $y$ into the equation for the line of best fit and can be approximated for any surfactant loading ratio $x$ per the equation $c^{*}=0.1767 x+49.203$. In Figures 3.14, 3.15, and 3.16, an assortment of images are displayed which represent the isotropic, biphasic, and nematic liquid crystal phases for various polymer/surfactant complexes. Interesting textures of the nematic liquid crystal phase formed via mechanical shearing will also be introduced. 


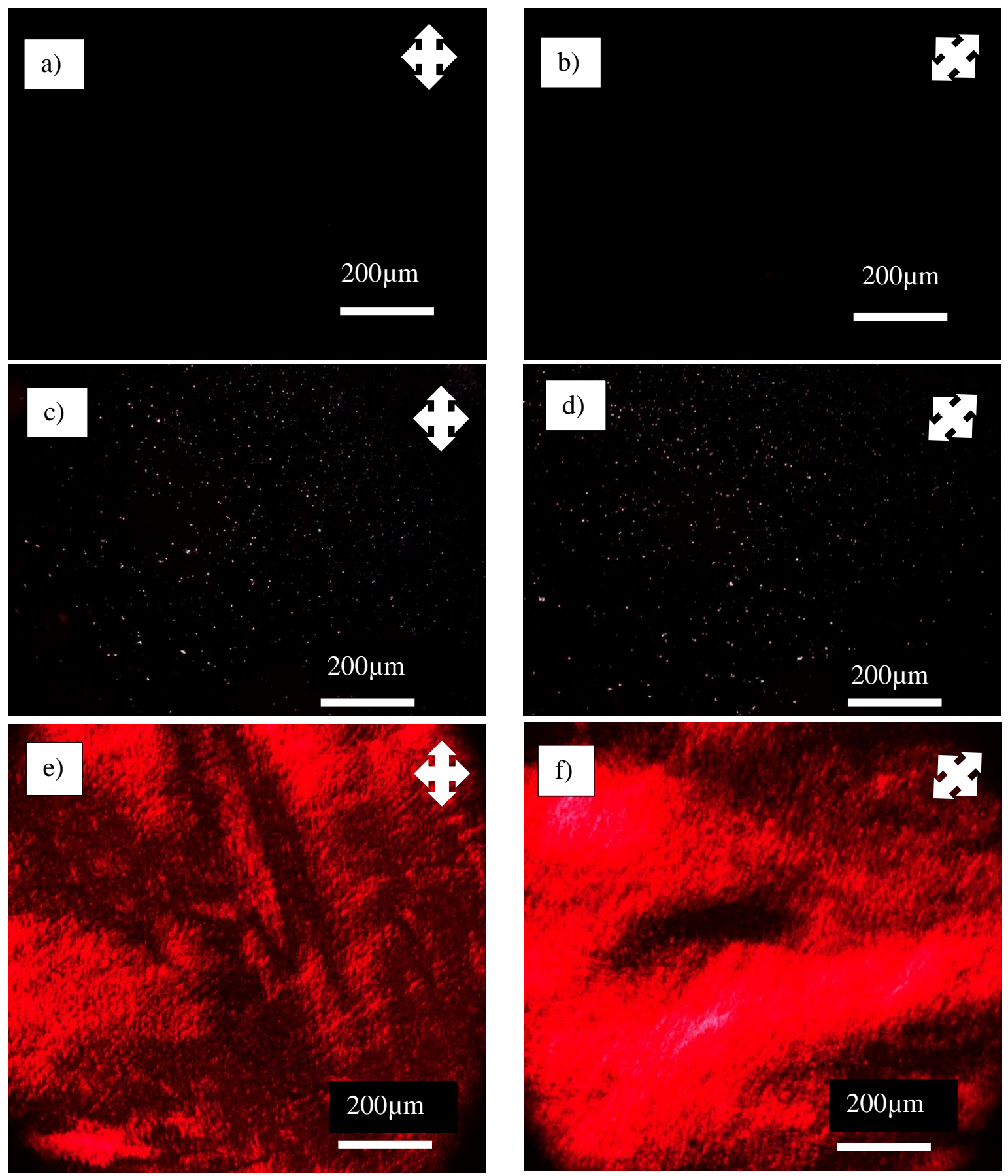

Figure 3.14: Illustration of the Nematic, Biphasic, and Liquid Crystal Phases before and after rotation of polarized light. a) Isotropic Phase; $\mathrm{C} 10$ complex at 52wt. \% concentration, 0 degrees rotation. b) Isotropic Phase; C10 complex at 52wt.\% concentration, 45 degrees rotation. c) Biphasic Phase; C10 complex at 56wt.\% concentration, 0 degrees rotation. d) Biphasic Phase; C10 complex at 56wt.\% concentration, 45 degrees rotation. Only slight birefringence occurs with respect to rotation of polarized light. e) Liquid Crystal Phase; C10 complex at 62wt.\%, 0 degrees rotation. f) Liquid Crystal Phase; C10 complex at 62wt.\%, 45 degrees rotation. 

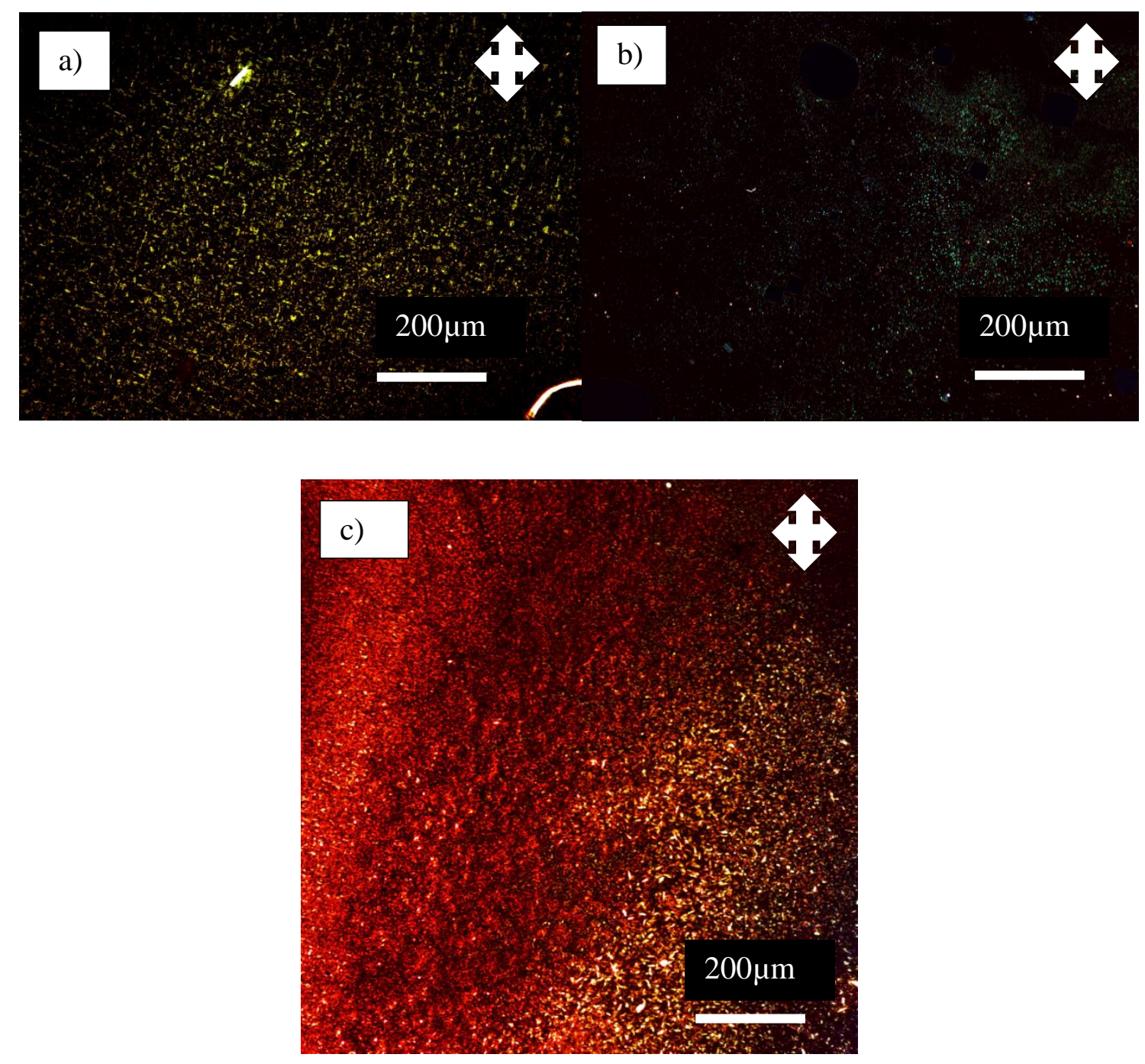

Figure 3.15: Other illustrations of the Biphasic Phase. a) Biphasic Phase;

mechanically sheared C16 complex at 52wt.\% concentration. b) Biphasic Phase; C16 complex at 53wt.\% concentration. c) Biphasic Phase; poly(3-butylthiophene-2, 5-diyl): CTAB complex at approximately $55 \mathrm{wt} . \%$ concentration. 

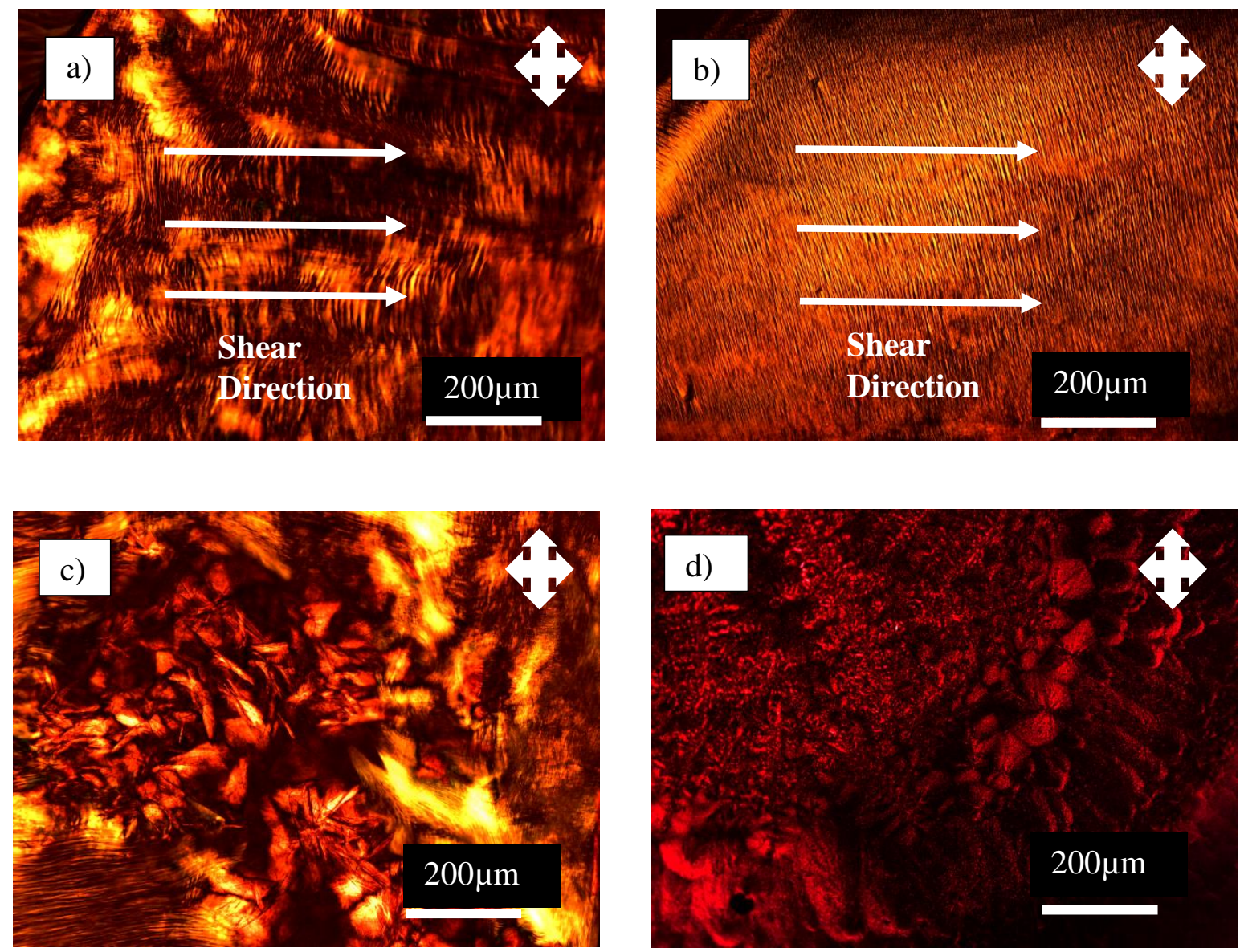

Figure 3.16: Depiction of other attainable liquid crystalline and solid film textures.

a) Nematic Liquid Crystal Phase; banded texture obtained via an applied shear force. b)

Nematic Liquid Crystal Phase; 60wt.\% C16 complex with banded texture obtained via

shearing. c) Nematic Liquid Crystal Phase; Schlieren texture obtained as a result of

defects forming, wherein brushes meet at a common defect point. d) Solid film of a C16 complex at $60 \mathrm{wt} . \%$ concentration with spherulites present in the right side of the image.

As a general rule, as the percentage of DHAB in the complex decreases, the degree of birefringence, and hence of the nematic liquid crystalline phase, increases. The differences in the extent of birefringence seen from polarized optical microscope images introduce a considerable amount of subjectivity, as birefringence can only be demarcated 
by the extent of contrast between black and red. In reality, the birefringence and length are not constant across an entire sample in a typical liquid crystal.${ }^{67}$ There will invariably exist regions of differing director orientation and length, and as such, some samples might display the Schlieren texture where dark regions referred to as brushes represent alignment parallel or perpendicular to the director ${ }^{67}$ The data gathered for the phase transition graph of Figure 3.13 was obtained in 2012 as part of a different study. Nevertheless, new data was acquired which roughly agrees with the trends of Figure 3.13. In Figure 3.17, the experimentally determined phase transition from the isotropic to nematic liquid crystalline phase is depicted via a line of best fit. No biphasic phases were observed for this particular study and it was assumed that there was an uncertainty of roughly $4 \mathrm{wt} . \%$ for all concentrations of the complex. Based on the trend seen in Figure 3.17, increasing the concentration of a solution will favor the formation of the nematic liquid crystalline phase, irrespective of the CTAB: DHAB surfactant composition. This tendency agrees with Onsager's Rigid Rod Theory in that the decrease in the orientational entropy of the polymer/surfactant entities varies directly with the concentration of the polymer/surfactant mixture. In simpler terms, greater crowding leads to less chain mobility, and in order to attain a lower Gibbs free energy, the chains selfassemble into a more ordered or liquid crystalline morphology, which raises packing entropy. Packing entropy is the result of increased disorder within the surrounding solvent when the polymer chains become increasingly ordered. The total entropy of the system (i.e. of polymer and solvent) increases despite the lowering of orientational entropy when the chains are aligned in the liquid crystalline phase. 


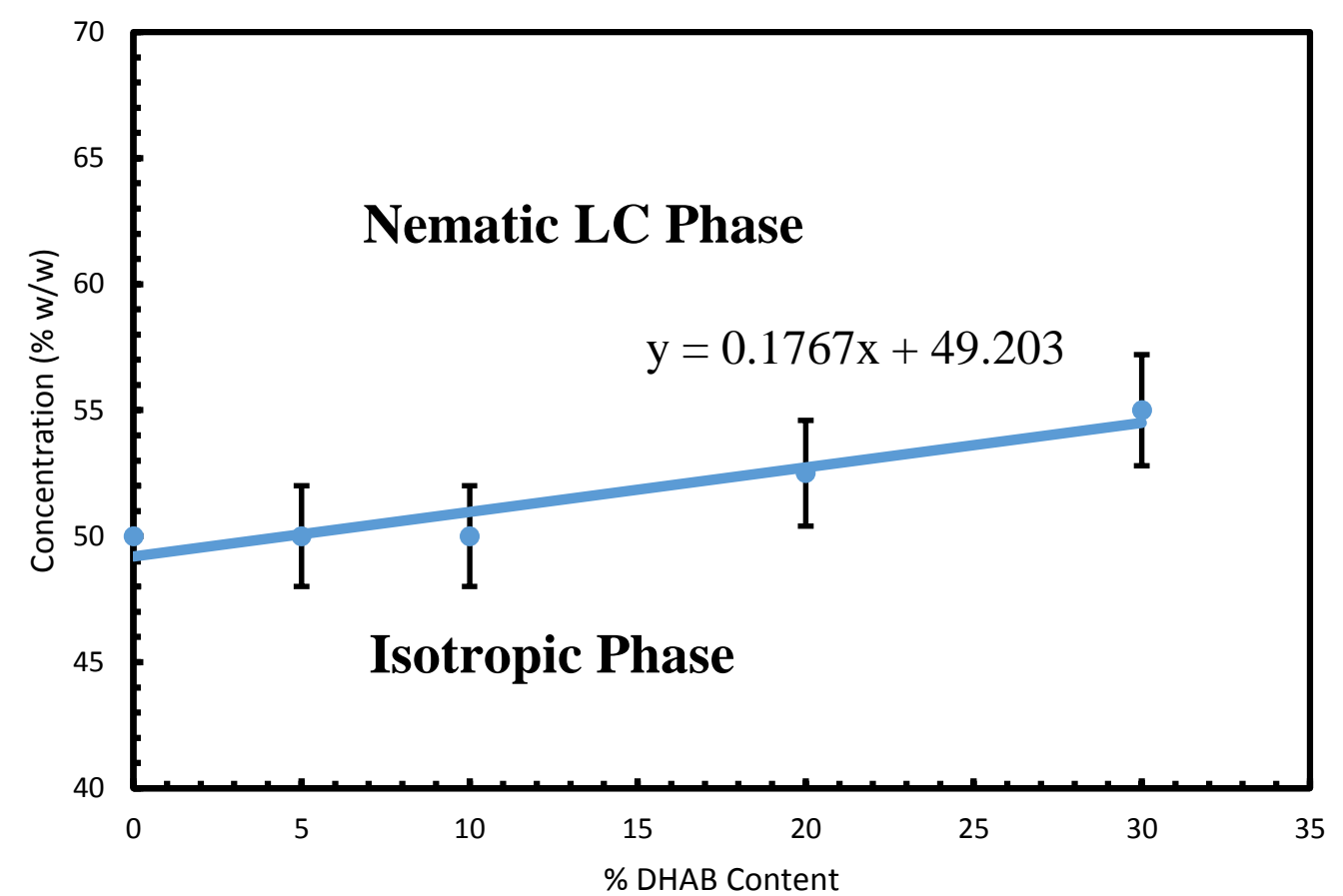

Figure 3.17: Experimentally determined phase transitions for P3KHT complexes of varying DHAB loading ratios.

The experimentally determined weight percent concentrations at which phase transitions occur for complexes with single tailed surfactants also agree with Onsager's Rigid Rod Theory. As the alkyl chain length of the single tailed surfactant increases, there is less total volume for the polymer chains to move. Increased steric repulsions result between adjacent surfactant units, since the longer alkyl chains occupy more space and inevitably become closer to each other in a system wherein the volume is fixed. As a consequence, the polymer chains must undergo a transition from the isotropic to liquid crystalline state so as to maximize positional entropy and to attain the most stable thermodynamic state. In essence, for a fixed concentration, a complex possessing longer single tailed surfactants will more heavily favor the liquid crystalline phase over the isotropic phase. In Figure 3.18, one can observe the effect of increasing surfactant alkyl 
chain length on the phase behavior of concentrated hydrogel solutions. No biphasic phase was observed, and an uncertainty of roughly $4 \mathrm{wt} . \%$ exists for all concentrations. The experimentally determined trend in Figure 3.18 is in direct agreement with Onsager's Rigid Rod Theory because as surfactant alkyl chain length is increased for a particular concentration, the liquid crystal nematic phase becomes more favorable.

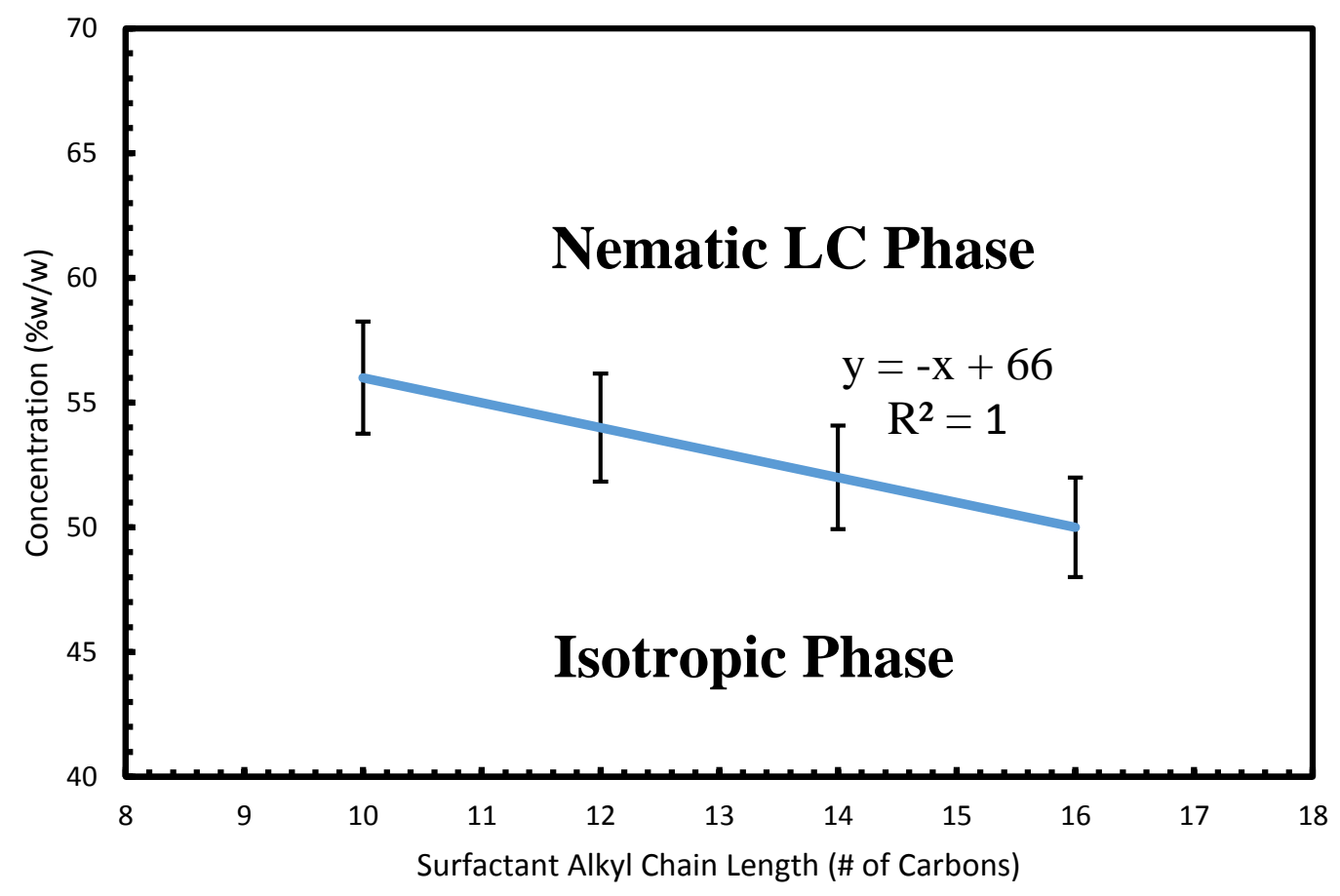

Figure 3.18: Experimentally determined phase transitions for P3KHT complexes of varying surfactant alkyl chain length. 


\subsection{Properties of Solid State Complexes}

Explicit trends demonstrating the relationship between crystallinity and surfactant architecture were observed in Differential Scanning Calorimetry (DSC) and X-Ray Diffraction (XRD) studies. In theory, increasing the DHAB loading ratio with respect to $\mathrm{CTAB}$ or decreasing the alkyl chain length of a single chain surfactant will hamper the formation of perfect crystallites, and will instead increase the proportion of imperfect crystallites. Many studies have demonstrated the influence of surfactant architecture on the solid state properties of conjugated polyelectrolyte complexes—in a study by Thunemann and coworkers, substantial increases in crystallinity were observed for systems containing more than eight or nine methylene groups. Systems with lower numbers of methylene groups (i.e. surfactants of shorter alkyl chain length) have lower melting enthalpies per mol of surfactant and yield more flexible polymer chains; as a result, flexible films with reversible elongation of approximately $28 \%$ may be obtained. ${ }^{59}$ X-Ray Diffraction data and DSC data can be utilized to indirectly deduce the extent of molecular ordering; X-Ray scattering intensity increased in direct proportion to charge density facilitated by long, single chain surfactants. ${ }^{58}$ Additionally, peak broadening indicative of greater amorphicity was observed to occur for systems with smaller diffracting domain sizes—-greater crystal lattice distortions and micro-strains were induced when certain surfactant architectures contributed to more compacted and less ordered morphologies. ${ }^{60}$ Thus, if one neglects all instrumental contributions to peak broadening within an X-Ray diffractogram, the full-width at half maximum (FWHM) should be directly proportional to the degree of amorphicity, and hence to the degree of coiled morphology. 
In Figure 3.19, it is evident that as the amount of DHAB with respect to CTAB is reduced, the extent of rod-like morphology is reduced, as is the crystallinity. The scattering peak seen at approximately $2.5 \AA^{-1}$ rises in intensity significantly from a $90 \%$ CTAB complex to a $100 \%$ CTAB complex - this trend agrees with the UV/Vis data seen in Figures 3.2 and 3.4 in that rod-like morphology is not favored for a 90\% CTAB complex (no red-shifting occurs). Moreover, a secondary diffraction peak is observed at approximately $5 \AA^{-1}$, which can be attributed to the formation of a secondary crystallite along the (200) orientation. The $100 \%$ CTAB complex is polycrystalline in nature based on XRD data.

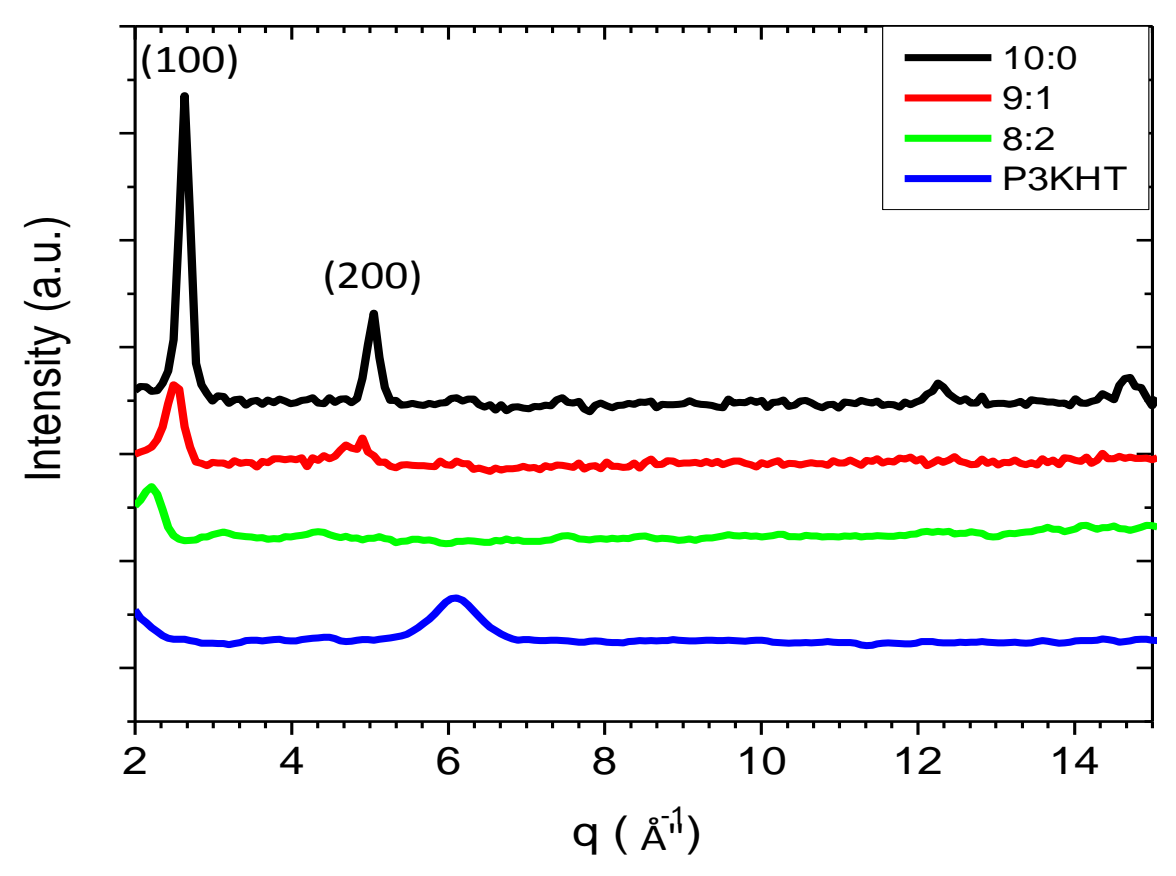

Figure 3.19: X-Ray Diffractogram of a P3KHT/Surfactant complex of varying DHAB concentrations. ${ }^{66}$ 
Data obtained via DSC reinforce the fact that crystallinity increases as a function of total CTAB content or as a function of surfactant alkyl chain length. In Figures 3.20 and 3.21, the melting temperature of the complexes progressively increases with respect to the aforementioned variables; moreover, the endothermic integrated peak area also increases. In simplistic terms, more energy must be supplied to a system with greater crystallinity in order to break apart the rigid, intercalated networks as compared to a system with a coiled morphology, wherein the enthalpic cost of bond breaking is substantially less.

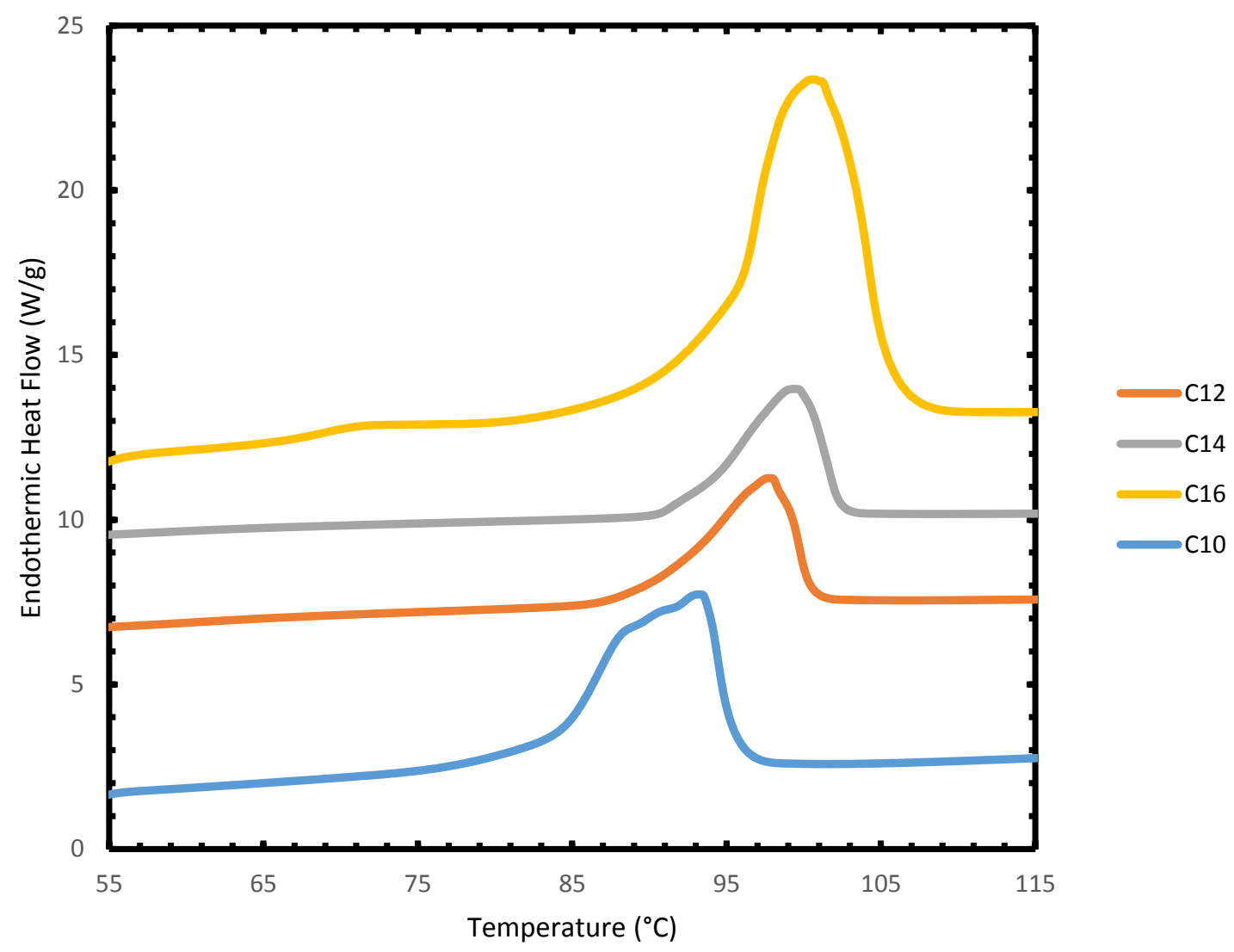

Figure 3.20: DSC spectra of P3KHT/Surfactant complexes of varying alkyl chain length. 


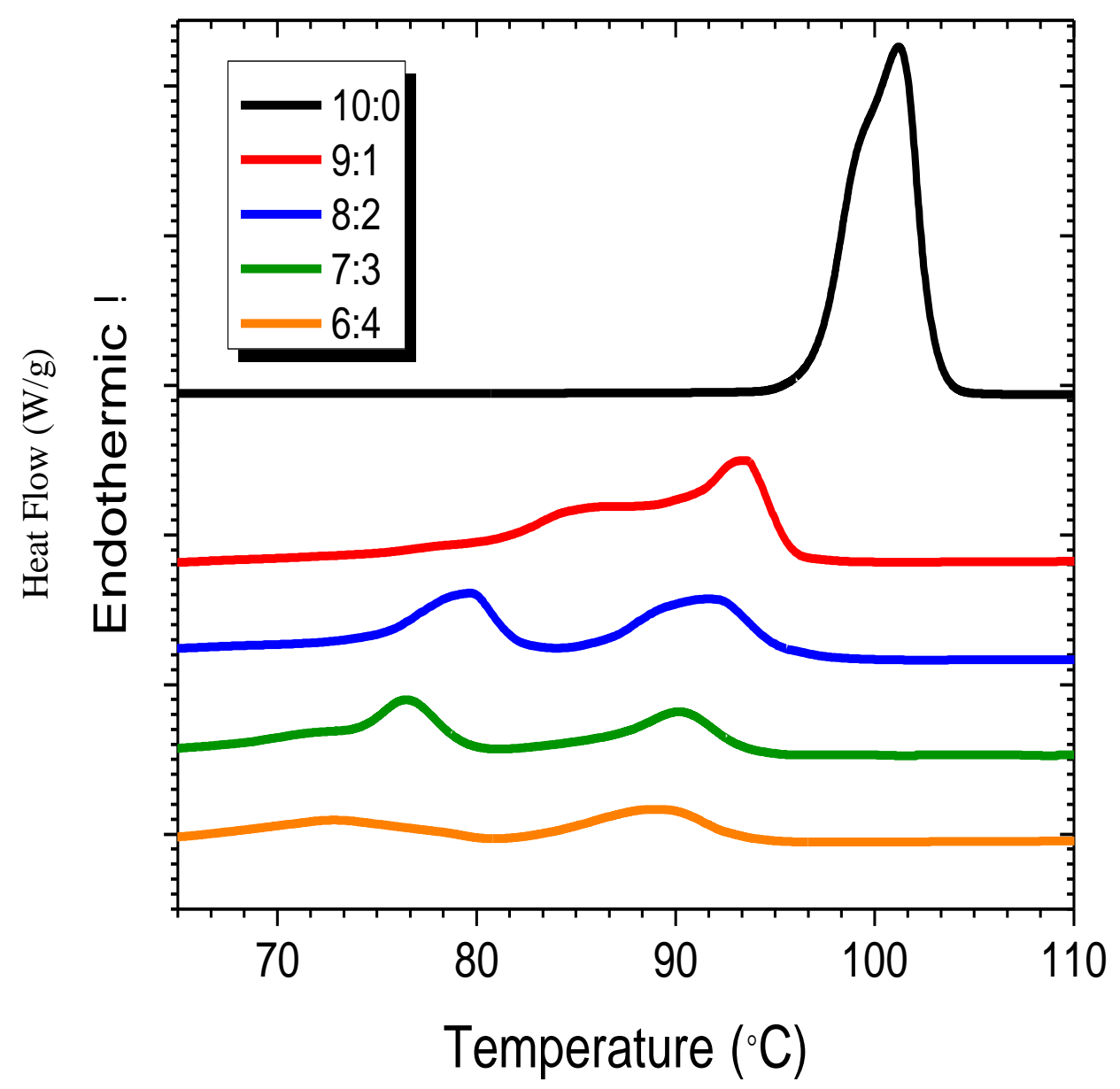

Figure 3.21: DSC spectra of P3KHT/Surfactant complexes of varying DHAB loading ratios. ${ }^{66}$

In Figure 3.20, there exist two melting peaks for complexes with greater than $10 \%$ DHAB content because crystalline impurities are likely to form in the more disordered coiled morphologies. Long range morphological order is compromised as DHAB content increases, and as a result, crystalline intercalated networks are disrupted, leading to a broadening of the melting regime(s). Another trend observable in Figure 3.21 is that the integrated peak areas, representative of the enthalpies of fusion, increase monotonically 
with $\mathrm{CTAB}$ content and hence with percent crystallinity. These developments allow for the DSC data to provide indirect evidence of an increase in the rod-like character of the P3KHT chains as DHAB is removed.

The extent of crystallinity of samples in the solid state may also be studied by means of optical and scanning electron microscopy. In the optical images to follow in Figure 3.22, one can notice the varied orientations of crystal fibers, which in some instances may aggregate to form spherulites. Spherulites are semicrystalline regions inside non-branched linear polymers; upon crystallizing from the melt, spherical domains can form if there are sufficient nucleation sites. The amount of nucleation sites may be controlled by intentionally adding crystallization promoters or seeds, which are typically plasticizers, fillers, dyes, salts, or acids. The sizes of the resulting spherulites are inversely proportional to the number of nucleation sites, as crowding between adjacent nucleating centers limits the size to which a spherulite may grow. 

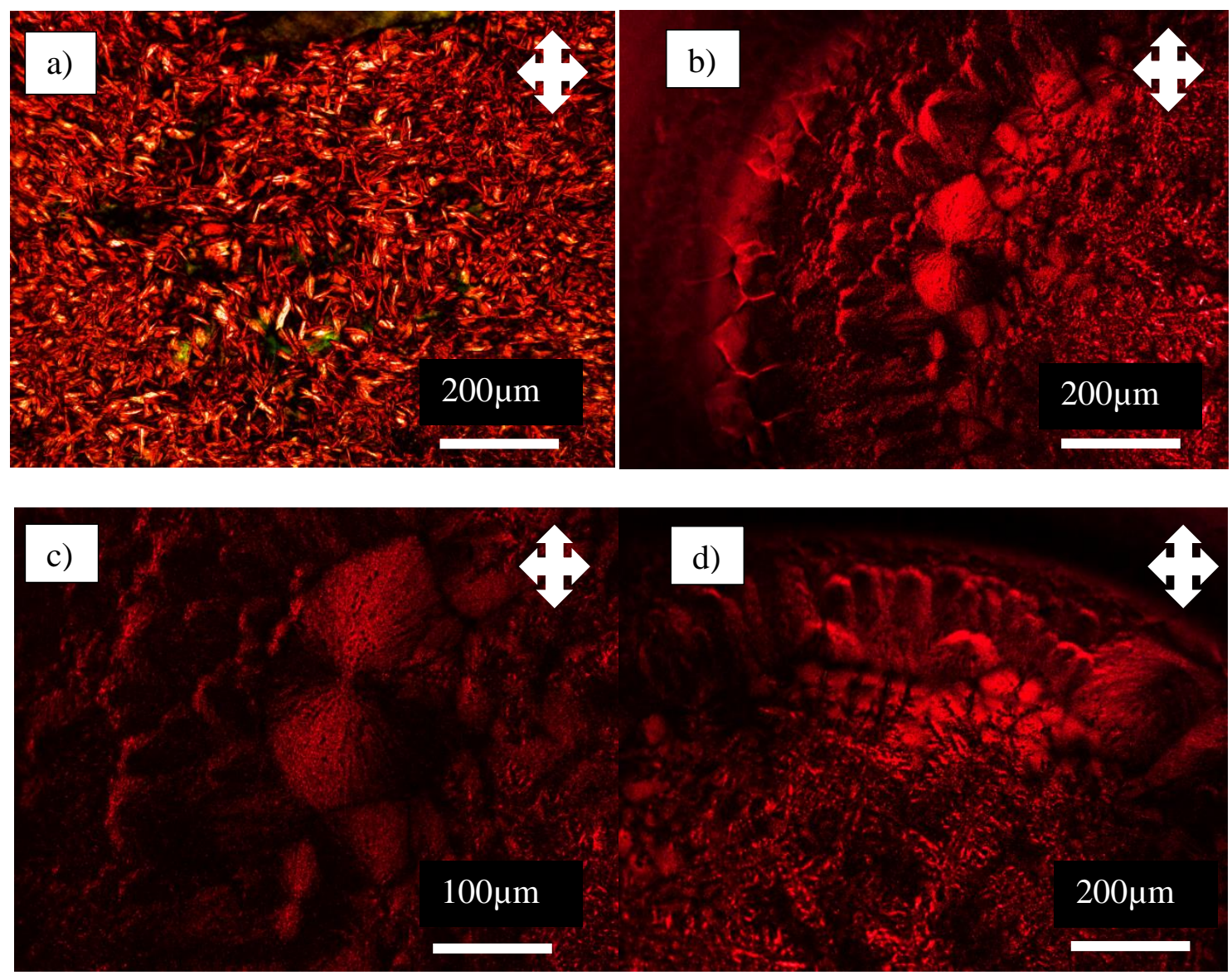

Figure 3.22: Polarized Optical Microscope (POM) Images of Dried

Polymer/Surfactant Films. a) A dried C16 complex. The orientation of the various crystallites varies throughout the film. b) A dried C16 complex. Large spherulites appear in the middle of the image. c) A zoomed in image of a dried C16 complex; one can notice the radial growth pattern of the crystals defining the spherulites. d) A dried C16 complex. Individual crystallites (lower half of image) and spherulites (upper half of image) define the texture of the dried film. 


\section{Future Work and Research Outlook}

Because an exhaustive list of suggested future experiments may be devised for investigating the role of surfactant architecture on P3KHT morphology, only a few feasible experiments will be herein discussed. While the UV/Vis, Fluorescence, XRD, and DSC data have provided indirect evidence of relating surfactant architecture to crystallinity and chain morphology, more direct evidence would be especially valuable to enhance the scientific validity of the research findings. Microscopic techniques will be imperative for providing visual evidence of the polymer and surfactant mixtures. The use of scanning electron microscopy (SEM) will not likely provide adequate resolution of the individual polymer chains intermixed with surfactant; it will therefore be recommended to use transmission electron microscopy (TEM) best elucidate chain morphology from a microscopy standpoint. While a conventional TEM can provide nanometer to subnanometer resolution, there is a significant risk of damaging a polymeric sample from a highly energetic electron beam. Moreover, sample preparation can prove troublesome and expensive, as samples must be less than or equal to $100 \mathrm{~nm}$. Nevertheless, under the correct instrumental conditions and with a carefully prepared sample, heavily desired images displaying fine detail of the polymer's interaction with the surfactant and the overall polymer morphology may be obtained. Diffraction mode may also be used on a TEM, wherein resulting diffraction spots or disk patterns directly represent the crystal structure of the sample. One can ascertain the degree of crystallinity from observing a diffraction pattern in that amorphous samples will scatter an electron beam to form a broad halo, whereas a highly crystalline specimen will scatter the beam into well-defined spots. Lastly, one may index a corresponding diffraction pattern to determine which 
crystallographic planes diffract, interatomic layer spacing, and the lattice parameter associated with a material.

More characterization methods also exist which can further enhance one's understanding of how surfactant architecture affects polymer chain morphology. The scanning tunneling microscope (STM) can provide atomic resolution images of surfaces. All samples must be conductive for STM use and much practice is needed in order to acquire high quality images, but once suitable images are obtained, precise surface structure and atomic arrangement may be determined. If operated under the correct conditions, STM can create a surface map of electron density wherein the cationic moieties (i.e. CTAB or DHAB) can be differentiated from the anionic moieties (P3KHT), thereby producing a colored map related to polymer and surfactant orientation. Advanced X-Ray scattering techniques such as SAXS would also be beneficial in elucidating polymer conformation in either dilute or concentrated solutions. The intensity of the scattering with respect to the scattering vector $q$ will reveal information related to polymer persistence length, radius of gyration, and overall morphology. While sample preparation may be tedious and while experiments may take several hours or more, SAXS can render invaluable data to the polymer scientist regarding how individual structures pack together in solid, liquid, or film samples ${ }^{61}$ SAXS is considered by many to be an ideal accompaniment to electron microscopy in that while electron microscopy provides localized nanostructural information, SAXS delivers an average of this information across an entire sample.$^{61}$ If SAXS were to be performed on all of the P3KHT/surfactant complexes mentioned in the previous sections, the validity of the 
UV/Vis data with respect to polymer bandgap and morphology could be more concretely assessed.

Another area worthy of study for this research endeavor is anisotropic fluorescence spectroscopy. In anisotropic fluorescence spectroscopy, the anisotropy of a particular fluorophore is related to the ratio of an unpolarized light component to total incident light intensity; emitted light from a polarized light source will become unpolarized after interacting with a fluorophore for a certain time period. The time necessary for the transition from polarized to unpolarized fluorescence is dependent upon the rotational diffusion, macromolecular size, and overall anisotropy of the fluorophore. ${ }^{62}$ Anisotropic fluorescence studies can thus be meaningful in that changes in the molecular size of polymers and other macromolecules can be monitored, as can fluorophore lifetimes. ${ }^{62}$ The decay rate of fluorescence anisotropy can be described mathematically per equation 7

$r(t)=r_{0} \exp (-t / \theta)$

where $r_{0}$ is the anisotropy at $t=0, t$ is time in seconds, and $\theta$ is the rotational correlation time. Factors such as resonance energy transfer between molecules and enhanced rotational motions will shorten the anisotropic lifetimes of the fluorophores. As the rotational correlation time $\theta$ is reduced, molecules are likely smaller, can rotate more readily, and are less strongly bound to other entities. As a result, the anisotropy of the system decreases in a smaller time period $r(t) \multimap$ one can therefore directly correlate the fluorescence anisotropy decay time $\mathrm{r}(t)$ to macromolecular conformation, size, and aggregation behavior. 
It should lastly be mentioned that a publication is pending related to the work of this thesis report. Upon acquiring satisfactory SAXS data for the liquid crystalline complexes of $\mathrm{C} 16, \mathrm{C} 14, \mathrm{C} 12, \mathrm{C} 10$, and 5, 10, 20, 30\% DHAB, enough proof will have been made to justify the influences of surfactant architecture on conformational changes of CPEs in the dilute, high concentration, and solid state regimes. The characterization data for complexes in the dilute and solid state regimes is reproducible, accurate, and trustworthy; the only challenge remaining is to successfully prepare SAXS samples and to acquire informative SAXS data to gain a better understanding of the complexes' conformations in the liquid crystalline (high concentration) state. Upon preparing the previous batch of SAXS samples, the liquid crystalline solutions unexpectedly dried upon shipment, and therefore were not in a useful form for acquiring meaningful SAXS data. By devising a new way to ship the SAXS samples and with collaboration with the researchers at Yale, strong hopes have been made to gather the desired data so that the paper in progress may become submitted and published. 


\section{Conclusions}

Demand has increased immensely for organic electronic devices, primarily because of their cheaper processing routes. Organic solar cells, light emitting diodes, and thin film transistors are a few of the many popular polymer electronic devices whose efficiencies rely heavily upon a control of the polymer chain morphology. In this work, the architecture of various cationic surfactants was analyzed, as was the effect of this architecture on tailoring polymer chain morphology. Through ionic interactions between the cationic moieties of the surfactant and the anionic moieties of the conjugated polyelectrolyte, it is possible for the polymer chains to undergo a transition in morphology from 'coil to rod' under the correct conditions of temperature, concentration, and ratio of surfactants, etc. In the dilute regime, it was found that the formation of rodlike structures is impeded by increasing the proportion of DHAB with respect to CTAB or by decreasing the alkyl chain length of a single tailed surfactant. When the polymer/surfactant complexes are of higher concentrations, the nematic liquid crystalline phase becomes more favored than the isotropic phase as the alkyl chain length of a single tailed surfactant increases. Conversely, when the proportion of DHAB increases with respect to $\mathrm{CTAB}$, more compacted structures form and the isotropic phase becomes favored over the nematic liquid crystalline phase. The critical concentration, $c^{*}$, at which a phase transition occurs varies with respect to the surfactant architecture of the complex. Lastly, solid state films of the polymer/surfactant complexes were characterized via DSC and XRD, wherein a complexes' melting temperature and percent crystallinity were observed to increase in direct proportion to surfactant alkyl chain length or to higher CTAB: DHAB ratios. A fundamental work has herein been presented that will prove vital 
for bettering the understanding of how one may use surfactant(s) to adjust the morphology of conjugated polyelectrolytes. By tuning the morphology of the conjugated polyelectrolytes, the efficiency and manufacturability of organic electronic devices may be enhanced considerably. 


\section{REFERENCES}

(1) Staudinger, H. “Macromolecular Chemistry: Nobel Lecture," 1953, 1-23

(2) Jerome, D., Ribault, M., Bechgaard, K. “Organic Superconductors," New Scientist, 1980, 104-106

(3) Norden, B., Krutmeijer, E., Vetenskapsakademien, K., "The Novel Prize in Chemistry, 2000: Conductive Polymers," Nobel Prize Advanced Information, The Royal Swedish Academy of Sciences, 2000, 11-56

(4) Jafri, R.I., Mishra, A.K., Ramaprabhu, S., "Polyaniline- $\mathrm{MnO}_{2}$ Nanotube Hybrid Nanocomposite as Supercapacitor Electrode Material in Acidic Electrolyte,” J. Mater. Chem., 2011, 21, 17601-17605

(5) Seyler, H., Subbiah, J., Jones, D.J., Holmes, A.B., Wing Ho Wong, W., “Controlled Synthesis of poly(3-hexylthiophene) in Continuous Flow,” Beilstein J. Org. Chem., 2013, 9: 1492-1500

(6) Rahman, A., Kwon, N.H., Won, M.S., Choe, E.S., Shim, Y.B. "Functionalized Conducting Polymer as an Enzyme-Immobilizing Substrate: An Amperometric Glutamate Microbiosensor for in Vivo Measurements,” Anal. Chem., 2005, 77 (15), 4854-4860

(7) Burroughes, J.H., Bradley, D.D.C, Brown, A.R., Marks, R.N., Mackay, R.H., Burns, P.L., Holmes, A.B., "Light Emitting Diodes Based on Conjugated Polymers," Nature 347, 1990, 539-541 
(8) Dai, L. "Charge Transfer Complexes between Polyacetylene Type Polymers and Iodine in Solution,” J. Phys. Chem., 1992, 96 (15), 6469-6471

(9) Deng, W., Liang, X., Wu, X., Qian, J., Cao, Y., Ai, X., Feng, J., Yang, H. “A Low Cost, All-Organic Na-Ion Battery Based on Polymeric Cathode and Anode," Scientific Reports 3, Article \#2671, June 2013

(10) Pratt, C., "Conducting Polymers," 1996

(11) Bhattacharyya, D., Howden, R.M., Borrelli, D.C., Journal of Polymer Science Part B: Polymer Physics, 1 Oct. 2012, Vol. 50, Issue 19, i-ii, 1329-1393

(12) Crystallography in Russia, International Union of Crystallography, Vol. 12. No. 2. Web. 6 May 2015.

(13) Pixshark Images. 'Doped Polyacetylene.' Pixshark.com-Image Galleries with a Bite! Web. 6 May 2015.

(14) Dai, L., Lu, J., Matthews, B., Mau, A., "Doping of Conducting Polymers by Sulfonated Fullerene Derivatives and Dendrimers,” J. Phys. Chem. B, 1998, 102 (21), 4049-4053

(15) Polymer Solutions Incorporated (PSI), “The U.S. Conductive Polymers Market to Reach U.S. \$1.6 Billion by 2017, According to a New Report by Global Industry Analysts, Inc.," Virtual Strategy Magazine. 12 April 2012. Web. 7 May 2015.

(16) Organic Electronics at IC Packaging Lab, “Organic Semiconductors,” CTI, 2013. Web. 7 May 2015. 
(17) Nguyen, C.A. "Layer-by-Layer Assembled Solid Polymer Electrolyte for Electrochromic Devices,” Chem. Mater., 2011

(18) Hogan, H., "Building Hybrid LEDs by Directly Writing Blended Polymers," Applied Physical Letters 2007

(19) Horden, J. “Conjugated Polyelectrolyte Solar Cell: Efficiency 8.4\%," HOT Communication, 2012

(20) Shim, Y.B., Azo Materials, "Conductive Polymers: Applications for Electronic Devices and Sensors," 11 June 2013. Web. 7 May 2015

(21) Llorens, E., Armelin, E., Perez-Madrigal, M.M., del Valle, L.J., Aleman, C., Puiggali, J., "Nanomembranes and Nanofibers from Biodegradable Conducting Polymers," Polymers, 2013, 5(3), 1115-1157

(22) Chang, L., Lademann, J.B.B., Meerholz, K., Moule, A.J., "Effect of Trace Solvent on the Morphology of P3HT: PCBM Bulk Heterojunction Solar Cells," Advanced Functional Materials, 2011, Vol. 21. Issue 10, 1779-1787

(23) Qiao, Q., McLeskey, J.T., Xie, Y., Joshi, P., Van, X., You, Y., Ropp, M., Galipeau, D. "Polymer Photovoltaics from All-Water Solution Processing," IEEE, 2008, 1-5

(24) Duarte, A., Pu, K.Y., Liu, B., Bazan, G.C., Chemistry of Materials, 2011, 23, (3), $501-515$

(25) Yang, X.N., Loos, J., Veenstra, S.C., Verhees, W.J.H., Wienk, M.M., Kroon, J.M., Michels, M.A.J., Janssen, A.A.,J., Nano Letters, 2005, 5, (4), 579-583 
(26) Inzlet, G., "Conducting Polymers: A New Era in Electrochemistry,” 2012, $2^{\text {nd }}$ Edition, 1-59

(27) Tanaka, F., Koga, T., Kaneda, I., Winnik, F.M., "Hydration, Phase Separation, and Non-Linear Rheology of Temperature Sensitive Water Soluble Polymers," J. Phys. Condens. Matter., 2011 Jul. 20, 23(28)

(28) Zhang, S., "Liquid Crystalline Polymers,” Lecture, April 2015

(29) Pelligra, C.I., Majewski, P.W., Osuji, C.O., "Large Area Vertical Alignment of ZnO Nanowires in Semiconducting Polymer Thin Films Directed by Magnetic Fields," Nanoscale, 2013, 5, 10511-10517

(30) Lucas, B., Trigaud, T., Pothier, A., Boucle, J., Ratier, B., "Simple Strategy to Tune the Charge Transport Properties of Conjugated Polymer/Carbon Nanotube Composites Using an Electric Field Assisted Deposition Technique,” Polymer International, Aug. 2014

(31) Kakade, M., Givens, S., Gardner, K., Lee, K.H., Chase, D.B., Rabolt, J.F., "Electric Field Induced Orientation of Polymer Chains in Macroscopically Aligned Electrospun Polymer Nanofibers,” J. Am. Chem. Soc., 2007, 129(10), 2777-2782

(32) Angmo, D., "UV/Vis Spectroscopy" Plastic Photovoltaics,” DTU Energy, 30 June, 2014. Web. 20 Apr. 2015

(33) Kang, S.J., Kim, Y.S., Kim, W.B., Kim, D.Y., Noh, Y.Y., “Conjugated Polymer Chain and Crystallite Orientation Induced by Vertically Aligned Carbon Nanotube Arrays," ACS Appl. Mater. Interfaces, 2013 Sep 25; 5(18): 9043-50 
(34) Skotheim, E., Elsenbaumer, R.L., Reynolds, J.R., Dekker, M., "Handbook of Conducting Polymers," Vol. 2, 1998, p. 225

(35) Brabec, C.J., Sariciftci, N.S., J.C., Hummelen, “Plastic Solar Cells,” Advanced Functional Materials, 2001, Vol. 11, Issue 1, 15-26

(36) Ates, M., Karazehir, T., Sarac, A.S., "Conducting Polymers and their Applications," Current Physical Chemistry, 2012, 2, 224-240

(37) Sharma, G.D., Mikroyannidis, J.A., Singh, S.P., "Efficient Bulk Heterojunction Solar Cells Based on D-A Copolymers as Electron Donors and $\mathrm{PC}_{70} \mathrm{BM}$ as Electron Acceptor,” 2012, Materials Chemistry and Physics, 135, 25-31

(38) Anderson, N.A., Hao, E., Xin, A., Hastings, G., Lian, T., "Sub-Picosecond Photoinduced Electron Transfer from a Conjugated Polymer to $\mathrm{SnO}_{2}$ Semiconductor Nanocrystals,”2012, Physica E. 14, 215-218

(39) Collins, B.A., Li, Z., Tumbleston, T., Gann, E., McNeill, C.R., Ade, H., “Organic Solar Cells: Absolute Measurement of Domain Composition and Nanoscale Size Distribution Explains Performance in PTB7: PC 71 BM Solar Cells," Advanced Energy Materials, 2013, Vol. 3, Issue 1, 1-13

(40) Tumbleston, J.R., Ma, W., Wang, M., Gann, E., Huang, F., Ade, H., “Domain Purity, Miscibility, and Molecular Orientation at Donor/Acceptor Interfaces in High Performance Organic Solar Cells: Paths to Further Improvement,” Advanced Energy Materials, 2013, Vol. 3, Issue 7, 864-872 
(41) Collins, B.A., "The Importance of Domain Size and Purity in High-Efficiency Organic Solar Cells,” Advanced Light Source: An Office of Science User Facility, Lawrence Berkeley National Laboratory, 26 Mar. 2013. Web. 20 Apr. 2015

(42) Zhang, S. "Liquid Crystalline Polymers,” Lecture. San Luis Obispo, CA. 15 April 2015

(43) Polymer Liquid Crystals Tutorial, "Main Chain Polymer Liquid Crystals," Case Western Reserve University, Mar. 2013. Web. 20 Apr. 2015

(44) Silva, E., Wang, Y., Zhu, Y., “Short Liquid Crystal Polymer Survey,” FPS 792: Polymer Introduction, 2010, North Carolina State University

(45) Rosenblatt, C., Shioda, T., Wen, B., “Continuous Nematic Anchoring Transition Due to Surface-Induced Semectic Order,” Phys. Rev. E. 67, 041706, 17 Apr. 2003

(46) Farrell, B., Zhang, T., Johnson, W., St. Lawrence, M., “The Processing and Assembly of Liquid Crystalline Polymer Printed Circuits” Auburn University Department of Engineering, 1996

(47) Jayaraj, K.J., Farrell, B., Noll, T.E., Larmouth, R.S., “A Low Cost Near-Hermetic Multichip Module Based on Liquid Crystal Polymer Dielectrics," Proceedings of SPIE-01/1996

(48) Iramis, “What is Measured in Small Angle X-Ray Scattering?” CEA. 15 Nov. 2013. Web. 20 Apr. 2015. 
(49) Kitamura, C., Tanaka, S., Yamashita, Y., Karikomi, M., "New Narrow-Bandgap Polymer Composed of Benzobis (1, 2, 5-thiadiazole) and Thiophenes,” J. Am. Chem. Soc., 1995, 117 (25), 6791-6792

(50) Chemistry Beta "How to Find the Band Gap for a Compound," Questions, Stack Exchange, 20 Apr. 2015. Web. 20 Apr. 2015

(51) Danesh, C.D., Starkweather, N.S., Zhang, S., "In Situ Study of Dynamic Conformational Transitions of a Water-Soluble Poly(3-hexylthiophene) Derivative by Surfactant Complexation,” The Journal of Physical Chemistry B, 2012, A-H

(52) Schwartz, J.A., Putyera, K., Contescu, C., Spicer, PT., "Bicontinuous Liquid Crystalline Nanoparticles," Encyclopedia of Nanoscience and Nanotechnology, $2004,881-892$

(53) Suh, K.Y., Kim, Y.S., Lee, H., J. Chem. Phys. 1998, 108, 1253

(54) Musking, N., Magaraphan, R., Coiai, S., Passaglia, E., "Effect of Surfactant Alkyl Chain Length on the Dispersion, and Thermal and Dynamic Mechanical Properties of LDPE/Organo-LDH Composites," Express Polymer Letters, Vol. 5, No. 5 (2011), 428-448

(55) Marquez, M., Kim, S., Jung, J., Truong, N., Teeters, D., Grady, BP., “Factors Affecting the Synthesis of Polymeric Nanostructures from Template Assisted Admicellar Polymerization," Langmuir, 2007, Sep. 25; 23(20): 10008-19 
(56) Onsager, L. "The Effects of Shape on the Interaction of Colloidal Particles," Annals New York Academy of Sciences, 1949, 51(4): 627-659

(57) PLC Virtual Textbook, "Liquid Crystal Phases," Case Western Reserve University, 2004. Web. 20 Apr. 2015

(58) Thunemann, A.F., "Polyelectrolyte-Surfactant Complexes (Synthesis, Structure, and Materials Aspects,” Prog. Polym. Sci. 27 (2002) 1473-1572

(59) Thunemann, A.F., "Nanostructured Dihexadecyldimethylammonium-Poly (1, 4phenylene-ethinylene-carboxylate): An Ionic Complex with Blue Electroluminescence,” Advanced Materials, Vol. 11, Issue 2, Feb. 1999, 127-130

(60) Barnes, P., Jacques, S., Vickers, M., “Sources of Peak Broadening,” Birkbeck College, University of London, 2006. Web. 20 Apr. 2015

(61) Reeves, R., "Materials for High Value Technologies," MacDiarmid Institute, Nov. 2014. Web. 21 Apr. 2015

(62) Horiba Scientific, Inc., "Fluorescence Application Notes: Steady State Fluorescence,” 2015. Web. 21 Apr. 2015

(63) Heo, J.H., Im, S.H., Noh, J.H., Mandal, T.N., et. al., "Efficient Inorganic-Organic Hybrid Heterojunction Solar Cells Containing Perovskite Compound and Polymeric Hole Conductors," Nature Photonics, 7, 486-491 (2013)

(64) Zeng, H., Zhu, X., Liang, Y., Guo, X., “Interfacial layer Engineering for Performance Enhancement in Polymer Solar Cells," Polymers, 2015, 7(2), 333372 
(65) Ehrentraut, H., "Overview of the Mesoscopic Theory of Liquid Crystals," Institut fur Theoretische Physik, 6 March 1997. Web. 7 May 2015

(66) Danesh, C.D., Zhang, S., Gopinadhan, M., Osuji, C.O., "Surfactant Induced MultiScale Assembly of a Water Soluble poly(3-hexylthiophene) Derivative,” 2011

(67) Case Western Reserve University, "Project Learning Tutorial: Virtual Textbook of Polymers and Liquid Crystals,” 2015. Web. 7 May 2015

(68) Berne, B.J., Pecora, R., “Dynamic Light Scattering: With Applications to Chemistry, Biology, and Physics,” Courier Dover Publications (2000), 24-53

(69) Grant, C.D., Schwartzberg, A.M., Smelted, G.P., et. al., "Characterization of Nanocrystalline and Thin Film $\mathrm{TiO}_{2}$ Solar Cells with poly(3-undecyl-2, 2'bithiophene) as a Sensitizer and Hold Conductor," Journal of Electroanalytical Chemistry, 2002, 522(1): 40-48

(70) Breeze, A.J., Schlesinger, Z., Carter, S.A., "Charge Transport in $\mathrm{TiO}_{2} / \mathrm{MEH}-\mathrm{PPV}$ Polymer Photovoltaics,” Phys. Rev. B, 2001, 64:1-9

(71) Ravirajan, P., Haque, S.A., Durrant, J.R., et. al., “The Effect of Polymer Optoelectronic Properties on the Performance of Multilayer Hybrid Polymer/TiO Solar Cells,” 2005, Adv. Funct. Mater. 15:609-18

(72) Kwong, C.Y., Djurisic, A.B., Chui, P.C., et. al., "Influence of Solvent on Film Morphology and Device Performance of poly(3-hexylthiophene): $\mathrm{TiO}_{2}$ Nanocomposite Solar Cells,” 2004, Chem. Phys. Lett., 384: 372-375 
(73) Qiao, Q., Xie, Y., McLeskey, J.J.T., “Organic/Inorganic Polymer Solar Cells Using a Buffer Layer from All-Water Solution Processing,” 2008, J. Phys. Chem. C 112:9912-16

(74) Gedde, U., "Polymer Physics," 1995, Springer-Science and Business Media, B.V., p. 118

(75) Lockwood, D., "Flexible Solar Cells Could Release Toxic Metals after Disposal," Chemical and Engineering News. 29 October, 2013. Web. 29 May, 2015

(76) Li, L. G., Lu, G. H., Yang, X. N., J. Mater. Chem. 2008, 18, 1984

(77) Osuji, C.O., Elimelech, M., Feng, X., Tousley, M.E., “Aligned Nanostructured Polymers by Magnetic-Field Directed Self-Assembly of a Polymerizable Lyotropic Mesophase,” ACS Appl. Mater. Interfaces, 2014, 6(22), 19710-19717

(78) Boudreau, D.M., "Shear Induced Orientation in Nematic Liquid Crystal Monodomains," Jan. 1, 2001. Doctoral Dissertations Available from Proquest. Paper AAI3027181

(79) Bae, S.C., Lin, Z., Granick, S., “Conjugated Polymers Confined and Sheared: Photoluminescence and Absorption Dichromism in a Surface Forces Apparatus," Macromolecules, Oct. 2005, 38(22)

(80) Zhang, S., Pelligra, C.I., Keskar, G., Majewski, P., Ren, F., Pfefferle, L., Osuji, C., "Liquid Crystalline Order and Magnetocrystalline Anisotropy in Magnetically Doped Semiconducting ZnO Nanowires,” 2011, ACS Nano, Vol. 5, No. 10, 83578364 
(81) Bagher, A.M., "Comparison of Organic Solar Cells and Inorganic Solar Cells," 2014, International Journal of Renewable and Sustainable Energy, 3(3): 53-58

(82) Kirschbrown, J., “Small-Angle X-Ray Scattering: A Concise Review,” 2007. Web. 5 June 2015

(83) Cullity, B.D., Stock, S.R., “Elements of X-Ray Diffraction,” 1956, Fig. 3-18, 101102 